\title{
Aplicações de semigrupos em sistemas de reação-difusão e a existência de ondas viajantes
}

\author{
Juliana Fernandes da Silva \\ DissertaÇÃo APRESENTADA \\ $\mathrm{AO}$ \\ Instituto de Matemática e Estatística \\ DA \\ Universidade DE SÃo PAUlo \\ PARA \\ OBTENÇÃO DO TÍTULO \\ $\mathrm{DE}$ \\ Mestre em CiênCIAS
}

Programa: Matemática Aplicada

Orientador: Prof. Dr. Sergio Muniz Oliva Filho

Durante o desenvolvimento deste trabalho a autora recebeu auxílio financeiro da CAPES e do CNPq.

São Paulo, agosto de 2010 


\title{
Aplicações de semigrupos em sistemas de reação-difusão e a existência de ondas viajantes
}

\author{
Este exemplar corresponde à redação \\ final da dissertação devidamente corrigida \\ e defendida por Juliana Fernandes da Silva \\ e aprovada pela Comissão Julgadora.
}

Banca Examinadora:

- Prof. Dr. Sergio Muniz Oliva Filho (orientador) - IME-USP.

- Prof. Dr. Antonio Luiz Pereira - IME-USP.

- Prof. Dr. Jair Silvério dos Santos - FFCLRP-USP. 


\section{Agradecimentos}

Agradeço ao meu orientador, Professor Sérgio Muniz Oliva Filho pela atenção e apoio durante a realização deste trabalho.

Aos professores que me ofereceram cursos de alto nível e com quem aprendi Matemática e, mais do que isso, tive bons exemplos de docência, nominalmente, Orlando Francisco Lopes, Frank Michael Forger e Pedro Antonio Santoro Salomão. Agradeço ainda aos professores com quem tive a oportunidade de desenvolver o programa PAE: Sônia Regina Leite Garcia, Pedro Aladar Tonelli

e Sérgio Muniz Oliva Filho. À professora Helena Maria Ávila de Castro pela atenção e cuidado durante o programa.

Agradeço aos professores que compuseram a banca de meu exame de qualificação, além de meu orientador, o professor Orlando Francisco Lopes e Antonio Luiz Pereira.

Agradeço aos professores Diogo Aguiar Gomes, Carlos Rocha, Waldyr Muniz Oliva, João Teixeira Pinto e Sofia Naique pela disponibilidade em meu período inicial em Lisboa. Agradeço ainda aos professores Pedro Girão, Lina Oliveira e João Santos por permitirem que eu assistisse seus cursos.

Ao IME, ao CNPq e à CAPES.

Agradeço aos membros da banca examinadora, os professores Antonio Luiz Pereira e Jair Silvério dos Santos, pelas importantes contribuições a este trabalho.

À minha família agradeço pelo apoio, pela dedicação e pela presença. Em especial, à minha mãe e ao meu pai, que sempre orientaram meus passos em um sentido precioso.

Ao Edgard. Pela companhia, pela paciência e por construir coisas comigo. 


\section{Resumo}

Sistemas de reação-difusão têm sido largamente estudados em diferentes contextos e através de diferentes métodos, motivados pela sua constante aparição em modelos de interação em contextos químicos, biológicos e ainda em fenômenos ecológicos. Neste trabalho nos propomos a estudar existência e unicidade - tanto do ponto de vista local como global - de soluções para uma classe de sistemas de reação-difusão acoplados, definidos em $\mathbb{R}^{2}$, utilizando como ferramenta a teoria de semigrupos de operadores lineares. Apresentamos dois importantes exemplos: o modelo de Rosenzweig-MacArthur e um particular caso da classe de equações $\lambda-\omega$. Para o primeiro obtemos um resultado de existência e unicidade global utilizando um método de comparação envolvendo sub e super-soluções. Investigamos ainda a existência de soluções de ondas viajantes periódicas através do teorema de Bifurcação de Hopf. Já para o caso da equação $\lambda-\omega$ obtemos a existência e unicidade de soluções, entretanto, a partir da aplicação da teoria de semigrupos de operadores lineares.

Palavras-chave: Sistemas reação-difusão, semigrupos de operadores lineares, ondas viajantes, ciclos limites. 


\section{Abstract}

Reaction-diffusion systems have been widely studied in a broad variety of contexts in a large amount of disctinct approaches. It is due firstly by their constant appearance in interaction models in disciplines such as chemistry, biology and, more specific, ecology. The aim of this thesis is to provide an existence-uniqueness result - both from the local as well as from the global point of view - for solutions of a particular class of coupled reaction-diffusion systems defined over $\mathbb{R}^{2}$. It is done applying the well established theory of semigroups of linear operators. Two remarkable examples of such systems are discussed: the Rosenzweig-MacArthur predator-prey model and a special case of $\lambda-\omega$ class of equations. For the former one, an existence and uniqueness result is obtained through a comparison method - based on the notions of lower and upper solutions. Moreover, we investigate the existence of periodic travelling wave solutions via a Hopf bifurcation theorem. For the $\lambda-\omega$ model another existence and uniqueness for solutions is obtained, on its turn, through the machinery obtained previously from the theory of semigroups for linear operators.

Keywords: Reaction-diffusion systems, semigroups of linear operators, travelling waves, limit cycles. 


\section{Sumário}

Agradecimentos $\quad$ i

$\begin{array}{lc}\text { Lista de Figuras } & \text { ix }\end{array}$

1 Introdução $\quad 1$

1.1 Considerações Preliminares . . . . . . . . . . . . . . . . . 1

1.2 Organização do Trabalho . . . . . . . . . . . . . . . . . . . . 2

2 Soluções de ondas viajantes para equações de reação-difusão 5

2.1 Equações de Reação-Difusão . . . . . . . . . . . . . . . . . . . . . . . . . . . 5

2.1 .1 Problema de Cauchy . . . . . . . . . . . . . . . . . . . . 6

2.1.2 Existência e unicidade de soluções . . . . . . . . . . . . . . . . . 9

2.1.3 Comportamento das soluções . . . . . . . . . . . . . . . . . . . . . 10

2.2 Ondas viajantes . . . . . . . . . . . . . . . . . . . . . . . . 13

2.2 .1 Definição e exemplos . . . . . . . . . . . . . . . . . 13

2.2.2 Classificação e existência de ondas . . . . . . . . . . . . . . . . 18

2.2 .3 Ondas viajantes periódicas em populações cíclicas . . . . . . . . . . . . 19

3 Teoria de semigrupos e o problema de Cauchy abstrato $\quad 21$

3.1 Teoria Espectral de Operadores Lineares . . . . . . . . . . . . . . . . . . . . . 21

3.1.1 Conceitos e resultados básicos da teoria espectral . . . . . . . . . . . . . . 21

3.1 .2 Operadores fechados . . . . . . . . . . . . . . . . . 24

3.1 .3 Operadores adjuntos, simétricos e auto-adjuntos . . . . . . . . . . 27

3.1 .4 Operadores dissipativos . . . . . . . . . . . . . . . . . . 30

3.2 Semigrupos de operadores lineares . . . . . . . . . . . . . . 33

3.2 .1 Definições e resultados básicos . . . . . . . . . . . . . . . . . 33

3.2.2 Os teoremas de Hille-Yosida e Lumer-Phillips . . . . . . . . . . . . . . . . . . 40

3.2.3 Operadores setoriais e semigrupos analíticos . . . . . . . . . . . . 47

3.2 .4 Potências fracionárias de operadores . . . . . . . . . . . . . . . . 57

3.3 Problemas de Cauchy lineares homogêneos e não homogêneos . . . . . . . . . . . . . 62

3.4 O problema de Cauchy semilinear . . . . . . . . . . . . . . . . . . . 69 
4 Sistemas de reação-difusão: aplicações de semigrupos, comparação e ondas viajantes

4.1 Existência e unicidade para o modelo envolvendo populações cíclicas $\ldots$. . . . . . . 77

4.2 O modelo de Rosenzweig-MacArthur e a existência de ondas viajantes periódicas . . 85

A Espaços de Sobolev e potências de Bessel $\quad 93$

A.1 Definições e resultados elementares . . . . . . . . . . . . . . . . . . . . . . 93

A.2 Potências de Riesz . . . . . . . . . . . . . . . . . . . . . . 95

A.3 Espaços de Sobolev . . . . . . . . . . . . . . . . . . . . . . . 96

A.4 Potências de Bessel . . . . . . . . . . . . . . . . . . . . 98

$\begin{array}{ll}\text { Referências Bibliográficas } & 101\end{array}$

$\begin{array}{lr}\text { Índice Remissivo } & 104\end{array}$ 


\section{Lista de Figuras}

2.1 Dinâmica da solução $u=u_{1}$ para $t=t_{1}$ e $t=t_{2}>t_{1}$ fixo. . . . . . . . . 14

2.2 Esboço de $f \ldots \ldots \ldots \ldots \ldots \ldots \ldots \ldots$

2.3 Retrato de fase para a equação 2.26 , caso $c=0 \ldots \ldots \ldots$

2.4 Esboço da variedade instável $W^{u}(0,0)$ para distintos valores de $c \ldots \ldots$. . . . . 17

$2.5 v_{+}=v_{-} \ldots \ldots \ldots \ldots \ldots \ldots \ldots \ldots \ldots \ldots \ldots \ldots \ldots \ldots \ldots \ldots \ldots \ldots \ldots$

3.1 Ilustração de uma conseqüência da inclusão $\{\lambda \in \mathbb{C}: \lambda>\|A\|\} \subset \rho(A) \ldots \ldots 25$

3.2 Setores $\Sigma_{\theta}(a)$ e $\Delta_{\theta}(a) \ldots \ldots \ldots \ldots \ldots \ldots \ldots$

4.1 Região invariante $R \operatorname{com} C=\frac{k \mu}{\sigma-\mu} \ldots \ldots \ldots \ldots \ldots \ldots$ 


\section{Capítulo 1}

\section{Introdução}

\subsection{Considerações Preliminares}

Sistemas de equações de reação-difusão são largamente utilizados para modelar situação de interação com duas componentes fundamentais: uma delas referindo-se ao espraiamento - de uma substância ou uma população - e a outra se referindo a reação das variáveis envolvidas a este espraiamento ou a forças adicionais. Por esta razão, esta classe de sistemas tem recebido especial atenção na modelagem de interações entre substâncias químicas distintas ou em modelos ecológicos - principalmente modelos do tipo predador-presa. Do ponto de vista matemático, um sistema de reação-difusão é um exemplo de um sistema parabólico semilinear e portanto, o entendimento das técnicas de investigação destes últimos é fundamental para o melhor entendimento de tais sistemas.

Diversas ferramentas são empregadas na literatura para o estudo de sistemas parabólicos semilineares. Algumas envolvem aspectos da teoria dos operadores, outras envolvem noções como princípios de comparação e, ainda, há aquelas dedicadas ao emprego dos métodos de computação numérica. Utilizaremos aqui técnicas associadas à teoria de semigrupos de operadores lineares. Assim sendo, com vistas ao melhor entendimento de sistemas de reação-difusão, o estudo da teoria destes semigrupos é fundamental. Em tempo, é no contexto desta teoria que generalizações de teoremas clássicos de existência e unicidade de equações diferenciais ordinárias (como Teorema de Picard e de Peano) podem ser encontradas (Teorema de Hille-Yosida e Lumer-Phillips).

Por outro lado, uma característica das soluções de sistemas de reação-difusão envolve a formação de padrões, ou seja, o aparecimento de estruturas regulares no sistema. Um padrão que tem recebido especial atenção na literatura por sua riqueza em descrição do fenômeno abordado é conhecido como solução de ondas viajantes. Dentre as propriedades mais importantes dos sistemas parabólicos nãolineares destacamos o fato de admitirem soluções desta classe.

Neste trabalho expomos inicialmente alguns aspectos fundamentais da teoria de sistemas de reação-difusão. Em particular, definimos o problema de Cauchy para este classe de equações e discutimos do ponto de vista qualitativo suas soluções. Em seguida, definimos e classificamos soluções do tipo ondas viajantes. Discutimos, através de alguns exemplos a importância desta classe de soluções, na medida em que os problemas em que estas aparecem podem ser tratados do ponto de vista de uma equação diferencial ordinária. Dentre os diversos tipos de ondas viajantes mencionamos as periódicas e não periódicas. As principais referências utilizadas nesta discussão 
são [1], [16], [24] e [36].

Em seguida, o trabalho dedica-se ao estudo e exposição dos principais fatos acerca de semigrupos de operadores lineares. Isto se deve ao fato de que estas equações podem ser entendidas como equações de evolução em algum espaço de funções, ou seja, para cada $t$ fixo, a incógnita $u(t)$ é uma função do espaço de funções considerado. Assim, investigar questões como existência e unicidade de soluções tangencia o problema análogo no contexto das EDO's, ainda que o espaço base, não seja mais um espaço vetorial Euclidiano. O objetivo então é associar a cada operador linear, ainda que não limitado, uma família de operadores a um parâmetro - $\mathrm{t}$ - que quando aplicados a um ponto $u_{0}$ - a chamada condição incial - nos fornece a solução do problema com tal condição $u_{0}$. Através de um conjunto de definições, exemplos e proposições, o trabalho visa tornar natural o entendimento de resultados como o Teorema de Hille-Yosida e Lumer-Phillips. Estes são então aplicados ao problema de Cauchy parabólico semilinear. O corpo de referências para este tópico é formado, basicamente, por [4], [7], [8], [17], [20], [23], [27] e [35].

Assim, através do emprego destes resultados, estudaremos o caso dos sistemas de reação-difusão em $\mathbb{R}^{2}$, mais precisamente, o exemplo das equações $\lambda-\omega$. A transposição dos resultados envolvendo semigrupos de operadores lineares para o caso particular dos sistemas de reação-difusão requer alguns resultados técnicos no âmbito dos espaços de Sobolev. Em prol da clareza do texto, estes resultados são organizados no apêndice ao trabalho. Finalmente, o tratamento do modelo de Rozensweig-MacArthur se dá de maneira distinta. Com o intuito de obtermos existência - local e global - e unicidade de soluções em alguma região do plano, utilizamos um método de comparação envolvendo sub e super-soluções do problema em questão. Ainda nesta caso, obtemos um resultado de existência de ondas viajantes através da aplicação de um teorema de bifurcação de Hopf, verificando a existência de ciclos limites para a equação diferencial ordinária associada. Aqui, os trabalhos de [5], [9], [11], [14], [33], [34] e [38] consituem a base das referências.

\subsection{Organização do Trabalho}

O capítulo 2 oferece uma discussão acerca dos sistemas de equações de reação-difusão e apresenta algumas de suas propriedades e exemplos. Mais ainda, discutem-se aspectos fundamentais das soluções de ondas viajantes, i.e., sua definição e classificação, bem como a interpretação dos fenômenos que descreve.

Já o capítulo 3 introduz de maneira detalhada a teoria dos semigrupos de operadores lineares.

É nesta ocasião que são expostos os elementos da Análise Funcional que serão utilizados adiante na formulação e demonstração de resultados como o teorema de Hille-Yosida e Lumer-Phillips. Que, por sua vez, serão úteis na obtenção de teoremas de existência e unicidade para problemas de Cauchy - lineares ou não.

Por fim, no capítulo 4, o arcabouço teórico desenvolvido no capítulo anterior é empregado na formulação de um resultado geral de existência e unicidade de soluções para um sistemas de reação-difusão de dimensão 2. Como ilustração deste resultado, um particular modelo de equações da forma $\lambda-\omega$ é exposto e discutido. Através de um método distinto - método de comparação abordamos o modelo de Rozensveig-MacArthur e obtemos a existência - local e global - e unicidade 
de soluções e, através do teorema de Bifurcação de Hopf, concluímos pela existência de soluções de ondas viajantes. 


\section{Capítulo 2}

\section{Soluções de ondas viajantes para equações de reação- difusão}

Neste capítulo discutiremos as definições e alguns resultados acerca das equações de reaçãodifusão e, em seguida, discutiremos um particular tipo de solução de tais equações, a saber, soluções do tipo ondas viajantes.

\subsection{Equações de Reação-Difusão}

Nas últimas décadas ${ }^{1}$, sistemas de equações de reação-difusão têm recebido uma atenção especial, motivada pelo seu constante aparecimento em modelos de fenômenos químicos e biológicos e, também pela riqueza da estrutura de suas soluções. Tais sistemas podem ser de vários tipos. Alguns são mais interessantes, outros nem tanto, a depender da área de estudo. As soluções normalmente representam a densidade de alguma substância, ou de uma população, em cada ponto do domínio espacial.

Estamos interessados em equações de reação-difusão da seguinte forma:

$$
u_{t}=D \Delta u+f(u)
$$

onde $u(x, t)$ representa a densidade ou concentração de alguma substância ou população, no tempo $t \geq 0$ e na posição $x \in \mathbb{R}^{n}$. Este não é o caso mais geral. A função $f$ pode, por exemplo depender das primeiras derivadas de $u$, de $x$ e ainda de $t$, mas neste trabalho restringiremos ao caso 2.1. $\Delta$ denota o laplaciano de $u$ com respeito à variável espacial $x$, e, em geral, representa um termo de difusão, com $D$ sendo uma constante positiva chamada constante de difusão. A parte de reação, representada pela função $f$ que em geral não é uma função linear, reflete um aumento (ou destruição) da quantidade de partículas. Mais ainda, a equação 2.1 é parabólica semilinear.

Uma equação de reação-difusão muito simples é a equação do calor - ou, de difusão -, dada por

$$
u_{t}=D \Delta u
$$

O nome da equação se dá pelo fato de que ela modela a distribuição de temperatura em todo o domínio em um instante $t$. Ela e suas variações aparecem em numerosos fenômenos de difusão.

\footnotetext{
${ }^{1}$ Ver, por exemplo, o survey em [16]
} 
A equação do calor é bastante simples pelo fato de que suas propriedades e o comportamento de suas soluções já terem sido amplamente estudados, contando com uma série de resultados e estudos acerca delas (ver [28], [21]). Uma propriedade muito simples e importante, ausente na maioria das equações de evolução mas, satisfeita pela equação 2.2 é a linearidade. É esta propriedade que permite a aplicação de técnicas importantes para a resolução de problemas em EDP's. Ainda assim, diversas questões interessantes e de difícil tratamento acerca das soluções de equações lineares podem ser levantadas.

Considere agora o seguinte exemplo de não linearidade: seja $f=u(1-u)$, um caso escalar e conhecido como não-linearidade logística de Fisher, e o problema de valor inicial - ou de Cauchy que é um tema a ser discutido com detalhes na próxima seção:

$$
\begin{array}{r}
u_{t}=\Delta u+u(1-u), \\
u(0)=u_{0} .
\end{array}
$$

Os pontos de equilíbrio são dados por $u=0$, que representa uma total extinção das espécies e é um ponto instável, e $u=1$, que representa uma total saturação e é exponencialmente estável. A solução exata para a EDO logística dada pode ser facilmente calculada e é da forma

$$
u=\frac{u_{0}}{\exp (-t)+u_{0}(1-\exp (-t))} .
$$

Apesar do exemplo dado ser bastante otimista no sentido de que sua solução foi facilmente encontrada e ainda numa forma exata, não é o que em geral ocorre no contexto das equações de evolução não-lineares. Neste último caso diversos métodos para representar soluções não são aplicáveis. Mais do que isso, em vários casos não conseguimos nenhuma forma exata da solução. Apesar disso, podemos fornecer algumas informações acerca deste tipo de equação e de suas soluções. O grande desafio do estudo das equações de evolução não-lineares é buscar técnicas analíticas qualitativas que nos permitam descrever soluções e prever seu comportamento. E é o que discutiremos na subseção 2.1.3.

\subsubsection{Problema de Cauchy}

Podemos adicionar à equação 2.1 uma condição inicial apropriada. Podemos, por exemplo, escolher uma função $u_{0}$ definida em algum domínio $\Omega \subseteq \mathbb{R}^{n}$ e considerarmos o problema de Cauchy

$$
\begin{gathered}
u_{t}=D \Delta u+f(u), \\
u(x, 0)=u_{0}(x) .
\end{gathered}
$$

Se $\Omega \neq \mathbb{R}^{n}$, então devemos especificar uma condição de fronteira em $\partial \Omega$. Seguem abaixo os 
principais exemplos de condições de fronteira:

(i) Condição de Neumann:

$$
\nabla u . \mathbf{n}=0, \quad x \in \partial \Omega, \quad t>0 .
$$

Onde $\mathbf{n}$ é o vetor normal exterior unitário de $\Omega$ no ponto $x \in \partial \Omega$.

(ii) Condição de Dirichlet:

$$
u(x, t)=b(x, t), \quad x \in \partial \Omega, \quad t>0 .
$$

Onde $b$ é uma função dada. O caso $b=0$ é chamado homogêneo.

(iii) Condição de Robin:

$$
\alpha(x, t) u+\beta(x, t) \nabla u . \mathbf{n}=b(x, t), \quad x \in \partial \Omega, \quad t>0 .
$$

Onde $\mathbf{n}$ e $b$ são como anteriormente e $\alpha$ e $\beta$ são funções dadas.

A terceira condição é também conhecida, por razões óbvias, como condição de fronteira mista. Observe que as condições acima são todas lineares na variável $u$. Apesar de também ser possível considerarmos condições não lineares, isto nos levaria a uma formulação mais difícil de ser estudada.

Quando modelamos um problema, se condições de fronteira são requeridas, suas determinações fazem parte do processo de modelagem. O comportamento das soluções será certamente afetado pela escolha de tais condições. Portanto, o mesmo cuidado tomado na hora de modelar uma equação de evolução deve ser também tomado na escolha das condições de fronteira.

Se impusermos à equação do calor, definida no domínio $\Omega \subseteq \mathbb{R}^{n}$, a seguinte condição inicial

$$
u(x, 0)=u_{0}(x),
$$

existem vários métodos de resolução deste problema de Cauchy, a saber, método da transformada de Fourier ou ainda método de separação de variáveis. Se $\Omega \neq \mathbb{R}^{n}$ então, como vimos, condições de fronteira também devem ser impostas. Uma vez que estamos interessados em um particular tipo de solução de problemas de reação-difusão, conhecida como ondas viajantes e que está definida em todo $\mathbb{R}^{n}$, citaremos [36] como referência para o estudo de problemas de Cauchy para este tipo de problemas em domínios ilimitados. Além disso, trataremos a seguir, como exemplo, a equação do calor definida em $\Omega=\mathbb{R}$. Tal problema é também conhecido como o problema da calor na barra infinita.

Exemplo 1 Considere o seguinte problema

$$
\begin{array}{r}
u_{t}=u_{x x},(x, t) \in \mathbb{R} \times(0, \infty), \\
u(x, 0)=u_{0}(x), x \in \mathbb{R},
\end{array}
$$

onde $u$ deve ser uma função limitada e definida em $C^{2}(\mathbb{R} \times(0, \infty)) \cap C(\mathbb{R} \times[0, \infty))$. O fato de 


\section{CAPÍTULO 2. SOLUÇÕES DE ONDAS VIAJANTES PARA EQUAÇÕES DE REAÇÃO-DIFUSÃO}

tomarmos u como sendo uma função limitada pode ser interpretado como uma condição de fronteira no infinito. Mais ainda, tal limitação garante a unicidade de solução de 2.10.

Usando o método de separação de variáveis, ou seja, procurando solução da forma

$$
u(x, t)=X(x) T(t)
$$

obtemos, substituindo em 2.10,

$$
\frac{X^{\prime \prime}}{X}=\frac{T^{\prime}}{T}=-\lambda,
$$

para alguma constante $\lambda$. Procuramos então funções $X$ e $T$ que sejam soluções, respectivamente, de

$$
X \in C^{2}(\mathbb{R}) \text { limitada, } X^{\prime \prime}=-\lambda X \text { em } \mathbb{R}
$$

$e$

$$
T \in C^{2}((0, \infty)) \cap C([0, \infty)) \text { limitada. }
$$

De onde segue que

$$
X(x)=A e^{\sqrt{-\lambda} x}+B e^{-\sqrt{-\lambda} x},
$$

onde $A$ e $B$ são constantes. Uma vez que $X$ deve ser limitada, $\operatorname{Re} \sqrt{-\lambda}=0, \lambda \in \mathbb{R}$ e $\lambda \geq 0$. Obtemos ainda que

$$
T(t)=C e^{-\lambda t}
$$

para alguma constante $C$.

Sendo assim, para cada $\lambda \geq 0$,

$$
u_{\lambda}(x, t)=\left(A_{\lambda} e^{i \sqrt{\lambda} x}+B_{\lambda} e^{-i \sqrt{\lambda} x}\right) e^{-\lambda t}
$$

Ou ainda, tomando $\lambda=\xi^{2} \operatorname{com} \xi \geq 0$,

$$
u_{\xi}(x, t)=\left(a_{\xi} e^{i \xi x}+b_{\xi} e^{-i \xi x}\right) e^{-\xi^{2} t}
$$

Uma vez que a família $\left\{u_{\xi}: \xi \geq 0\right\}$ não é enumerável, não podemos aplicar o Princípio da Superposição. Uma alternativa seria procurar soluções da forma

$$
u(x, t)=\int_{0}^{\infty} u_{\xi}(x, t) d \xi
$$

que pode ainda ser escrita como

$$
u(x, t)=\frac{1}{\sqrt{2 \pi}} \int_{-\infty}^{\infty} g(\xi) e^{i \xi x} e^{-\xi^{2} t} d t
$$


Impondo a condição inicial obtemos

$$
g(\xi)=\frac{1}{\sqrt{2 \pi}} \int_{-\infty}^{\infty} u_{0}(x) e^{-i \xi x} d x
$$

que é uma função conhecida como transformada de Fourier de $u_{0}$ (ver A.1).

A simplicidade em encontrar soluções neste caso, como foi mencionado anteriormente, se deve em grande parte ao fato de que 2.2 é uma equação linear. O mesmo não ocorre no caso não-linear. Na equação 2.1, tomando $f$ não-linear, nenhum dos métodos citados acima resolvem o problema de Cauchy.

De fato, dado, por exemplo, o seguinte problema não-linear e com condições de fronteira,

$$
\begin{array}{r}
u_{t}=u_{x x}-u u_{x}, \quad x \in(0, L), \quad t>0 \\
u(0)=0, u(L)=1,
\end{array}
$$

nenhuma das técnicas citadas acima é aplicável. Porém, argumentos qualitativos nos dão informações acerca do comportamento das soluções de 2.12. Podemos dizer, por exemplo, que se $u \equiv u_{0}$ é um ponto de equilíbrio da equação, então ele é assintoticamente estável. A demonstração deste fato e o significado da expressão assintoticamente estável serão feitas na subseção concernente ao comportamento das soluções.

Ou seja, embora neste último exemplo talvez seja impossível encontrar uma solução (na forma fechada ou em expansão), existem meios de se obter informações acerca da equação, bem como acerca do comportamento, existência e forma de suas soluções. Este tema será discutido adiante.

\subsubsection{Existência e unicidade de soluções}

Dizemos que o problema 2.5 está bem posto se ele possui uma única solução e esta depende continuamente da condição inicial. Consideraremos agora aspectos da boa colocação do problema.

Por existência local de soluções entendemos a existência de soluções do problema de Cauchy para uma pequena vizinhança em $t$ da condição inicial. Já o termo existência global é reservado para problemas cujas soluções existem para qualquer $t \in \mathbb{R}$.

No contexto das equações de reação-difusão, um ponto fundamental é que são vistas como equações de evolução em algum espaço de funções, ou seja, para cada $t$ fixo, $u(t)$ é alguma função do espaço dado. Sendo assim, a análise da teoria de existência e unicidade de soluções do problema de valor inicial é semelhante ao caso das EDO's porém o espaço base, não é mais um espaço vetorial de dimensão finita. Teoremas de existência como Picard e Peano são de grande relevância no contexto das EDO's, já no caso das EDP's - lineares - contamos com teoremas como o de Hille-Yosida e generalizações.

Uma arcabouço teórico poderoso no estudo da teoria de existência de soluções para equações de reação-difusão - na verdade, de EDP's, mas neste trabalho nos restringimos ao estudo deste particular tipo de equação - é a Análise Funcional, mais precisamente o estudo dos espaços de funções. Esta discussão, por ser o foco deste trabalho, será feita com detalhes no capítulo 3. 


\subsubsection{Comportamento das soluções}

A teoria qualitativa, ou geométrica, das equações diferenciais ordinárias foi efetivamente estudada por Poincaré e Liapunov (ver [25], [37]). E esta mesma teoria tem guiado o estudo das equações semilineares parabólicas - em particular, das equações de reação-difusão - quando questões acerca do comportamento global de suas soluções são levantadas.

Então, dada uma equação de reação-difusão uma análise muito importante a ser feita é a do comportamento de longo prazo das soluções. Pode-se querer saber, por exemplo, se as soluções convergem para um equilíbrio ou se irão se comportar de maneira periódica, aperiódica ou caótica. Alguns destes regimes são capazes de dominar o comportamento das soluções em uma certa vizinhança. Dentre as soluções com tais regimes, o tipo mais simples é o equilíbrio que é estável mesmo sob pequenas perturbações. Portanto, dado um problema de reação-difusão, é importante encontrar e classificar seus possíveis equilíbrios de acordo com suas propriedades de estabilidade.

Para muitos sistemas não-lineares a localização de um equilíbrio é um problema abordado somente qualitativamente. Se parâmetros na equação podem ser variados, o aparecimento e desaparecimento de equilíbrio pode ser estudado via técnicas da teoria de bifurcação. Por se tratar de uma teoria muito ampla e por escapar ao escopo do trabalho, nos restringimos à bifurcação de Hopf. Para uma abordagem mais geral, sugerimos [11]. A questão da estabilidade das soluções também está relacionada com a teoria de bifurcação. Um particular caso desta relação será discutido no capítulo 4 e, por enquanto, nos limitamos a definições e exemplos relacionados com a estabilidade de uma solução de equilíbrio.

Considere o seguinte problema:

$$
u_{t}=\Delta u+f(u), \quad x \in \Omega \subseteq \mathbb{R}^{n}, t>0,
$$

com condições de fronteira apropriadas caso $\Omega \neq \mathbb{R}^{n}$. Suponha que $u_{0}(x)$ seja um equilíbrio de 2.13. Nestas condições temos as seguintes definições - ou, classificação de $u_{0}(x)$ :

Definição 1 Dizemos que $u_{0}$ é um equilíbrio estável se, para qualquer vizinhança $V$ de $u_{0}$, existe uma vizinhança $W \subseteq V$ tal que qualquer solução de 2.13 que dependa do tempo e comece em $W$, permanece em $V$ para todo $t>0$. Além disso, se todas estas soluções, começando em $W$ convergem para $u_{0}$ quando $t \rightarrow \infty$, dizemos que $u_{0}$ é um equilíbrio localmente assintóticamente estável.

Definição 2 Dizemos que $u_{0}$ é um equilíbrio instável se, para qualquer vizinhança $V$ de $u_{0}$, existir uma solução de 2.13 e comece em $V$, mas que saia de $V$ quando $t \rightarrow \infty$.

As definições acima podem ser dadas mais precisamente e em termos de uma métrica ou norma em um espaço de funções que contenha as soluções. É o que faremos agora mas de maneira um pouco superficial e com a única finalidade de motivar discussões que serão feitas no capítulo 3.

Ainda com as notações acima, defina $u(x, t)=u_{0}(x)+v(x, t)$, onde $v$ é uma pequena perturbação 
do equilíbrio $u_{0}$, ou seja, $v(x, 0)$ é arbitrariamente pequeno. Substituindo em 2.13 obtemos

$$
v_{t}=\Delta v+f_{u} v
$$

Se condições de fronteira foram aplicadas à $u$, condições apropriadas de fronteira também deverão ser aplicadas a $v$. A equação acima, sujeita a qualquer condição de fronteira, é linear e pode ser escrita da seguinte forma:

$$
v_{t}+A v=0
$$

onde $A$ é um operador linear definido em um conjunto de funções apropriado, satisfaz condições de fronteira possivelmente impostas e $-A$ é dado por $\Delta+f_{u}$.

O operador laplaciano pode ser definido em diversos espaços como sendo um operador linear $\Delta: X \longrightarrow X$, ou seja, existe uma certa arbitrariedade na escolha do espaço de funções $X$. Apesar disso, $\Delta$ não é um operador contínuo (i.e., limitado) e está, portanto, definido em domínio $D(\Delta)$, que, se bem escolhido, será denso em $X$.

Com intuito de definir estabilidade em termos de normas em um espaço normado, consideramos $\Delta$ de modo que o espaço $X$ seja de Banach e que $D(A)$ seja um subconjunto denso de $X$. Veremos mais adiante que, usualmente, este pode ser o $L_{2}(\Omega)$ ou algum espaço chamado de Sobolev, a ser discutido mais adiante neste trabalho.

Reformulando as definições acima, ainda com as mesmas notações, temos

Definição 3 Dizemos que $u_{0}$ é um equilíbrio estável se, para toda constante positiva $M$, existir uma constante $N$ com $0<N \leq M$ tal que $\left\|u(x, 0)-u_{0}(x)\right\|=\|v(x, 0)\| \leq N$ implica que $\left\|u_{0}-u\right\|_{X}=$ $\|v\|_{X} \leq M$ para todo $t>0$. Se além disso, $\|v\|_{X} \rightarrow 0$, quando $t \rightarrow \infty$, dizemos que $u_{0}$ é localmente assintoticamente estável.

Definição 4 Dizemos que $u_{0}$ é um equilíbrio instável se $u_{0}$ não for estável.

Existem resultados muito importantes que nos dão informações acerca da estabilidade de uma solução de equilíbrio de uma equação de reação-difusão. Apresentaremos um deles agora, em uma formulação bastante simples e que será generalizada no capítulo seguinte.

Teorema 1 Sejam $\Omega$ um compacto e o problema 2.13 com a condição de fronteira

$$
\left.u\right|_{\partial \Omega}=C,
$$

onde $C$ é uma constante e $\partial \Omega$ denota a fronteira de $\Omega$. Então, o equilíbrio $u_{0}$ é assintoticamente estável se, e somente se, existir $\beta>0$ tal que $\operatorname{Re}(\lambda) \geq \beta$, para todo $\lambda$ tal que $(\lambda I-A)^{-1}$ não seja uma função limitada e invertível ${ }^{2}$ em $\Omega$.

\footnotetext{
${ }^{2}$ Ao conjunto de tais $\lambda$ 's chamaremos o espectro de $A$. Este conjunto receberá grande atenção no que segue neste trabalho e será mencionado em exemplos seguintes.
} 
Não faremos a demonstração agora, uma vez que uma versão mais geral do mesmo teorema será discutida no capítulo 3. Segue abaixo um exemplo de problema de reação-difusão, para o qual gostaríamos de determinar o comportamento das soluções na vizinhança de um ponto de equilíbrio, mas que abordaremos apenas de maneira qualitativa. Mais precisamente, usaremos o teorema acima afim de estudar o comportamento da função a longo prazo.

Exemplo 2 Considere o seguinte problema, em $\Omega=[0,10]$

$$
\begin{array}{r}
u_{t}=u_{x x}+f(u), \quad x \in(0,10), \quad t>0 \\
u_{x}(0)=u_{x}(10)=0, \quad t>0
\end{array}
$$

onde $f$ é uma função de classe $C^{\infty}$ e possui $u_{0}$ como raiz. Claramente $u \equiv u_{0}$ é um ponto de equilíbrio. Linearizando em $u \equiv u_{0}$, temos

$$
\begin{array}{r}
v_{t}=v_{x x}+f_{u}\left(u_{0}\right) v, \quad x \in(0,10), t>0 \\
v_{x}(0)=u_{x}(10)=0, t>0 .
\end{array}
$$

Neste caso, o operador $A: D(A) \rightarrow X$ é dado por

$$
A v=-v_{x x}-f_{u}\left(u_{0}\right) v
$$

e seu dominio é dado pelo conjunto de funções em $H^{2}(\Omega) \cap H_{1}^{0}(\Omega)$, e, portanto, $v(0)=v(10)=0$. Tomamos $X=L_{2}(\Omega)$ e usamos a norma de $X$. Como queremos encontrar o espectro de $A$, devemos resolver o seguinte problema

$$
\begin{array}{r}
-\phi_{x x}-f_{u}\left(u_{0}\right) \phi, \\
\phi_{x}(0)=\phi_{x}(10)=0 .
\end{array}
$$

Como soluções obtemos as seguintes autofunções, com seus respectivos autovalores

$$
\phi_{n}=\cos \frac{n \pi x}{10} \text { e } \lambda_{n}=-f_{u}\left(u_{0}\right)+\frac{n^{2} \pi^{2}}{100}, \quad n=1,2,3 \ldots
$$

Então, de acordo com o teorema $1, u \equiv u_{0}$ será um equilíbrio assintoticamente estável, se $f_{u}\left(u_{0}\right)<$ $\frac{n^{2} \pi^{2}}{100}$, para $n=1,2, \ldots$, ou seja, se $f_{u}\left(u_{0}\right)<\frac{\pi^{2}}{100}$. Caso $f_{u}\left(u_{0}\right)>\frac{\pi^{2}}{100}$, então $u \equiv u_{0}$ será um equilíbrio instável.

O exemplo não linear dado na subseção de problemas de Cauchy pode ser analisado também através do teorema acima. Basta observar que, se $u \equiv u_{0}$ é um ponto de equilíbrio da equação 2.13 , então ele satisfaz a equação

$$
\left(u_{0}\right)_{x}=\frac{u_{0}^{2}+\alpha}{2},
$$

para alguma constante real $\alpha$. Em seguida, deve-se encontrar uma expressão para $u_{0}$ e a equação 
linearizada em $u_{0}$, e depois é só proceder como no exemplo anterior.

Um comportamento bastante interessante de soluções de sistemas de reação-difusão envolve a formação de padrões, que consiste na aparição de estruturas regulares no sistema após um certo tempo. Tais padrões podem ser do tipo estáticos ou dinâmicos. Existem diversos tipos de padrões envolvidos em sistemas de reação-difusão em duas dimensões. Um particular tipo é o chamado padrão oscilatório que envolve, em particular, uma formação de padrão conhecida como ondas viajantes. Uma das propriedades mais importantes de sistemas parabólicos não-lineares é o fato de admitirem soluções deste tipo.

\subsection{Ondas viajantes}

Muitos fenômenos em contextos físicos e biológicos podem ser modelados por ondas viajantes. Como exemplos, podemos citar reações químicas oscilatórias e impulsos nervosos. Este tipo de solução faz parte de uma classe muito importante de soluções de equações de reação-difusão. O que se deve principalmente ao fato de que problemas deste tipo podem ser tratados como equações diferenciais ordinárias. O que não implica que o problema se torne trivial.

\subsubsection{Definição e exemplos}

Estaremos interessados, principalmente no capítulo 4, em um particular tipo de ondas viajantes, a saber, ondas viajantes periódicas. Uma característica interessante deste particular tipo de soluções consiste na sua similaridade entre o tempo e o espaço. Mais precisamente, se olharmos o sistema em tempo fixo $t$ por toda a dimensão espacial, observaremos o mesmo comportamento caso tivessemos fixado um ponto $x$ no espaço e verificado sua evolução durante o tempo - supondo que $x \in \mathbb{R}$. Em ambos os casos, teremos um comportamento periódico. A distância entre os picos periódicos representa o comprimento de onda das ondas viajantes no primeiro caso (ao longo do espaço) e a frequência para o segundo caso (ao longo do tempo).

Se uma solução $u(x, t)$ representa uma onda viajante, a forma da solução será a mesma para qualquer tempo $t$ e a velocidade de propagação desta tal forma será constante, e denotada por $c$. Formalmente, uma solução do tipo onda viajante é da forma

$$
u(x, t)=u(\mathbf{n} \cdot x+c t)=v(z), \quad z=\mathbf{n} \cdot x+c t,
$$

onde $\mathbf{n}$ é um vetor unitário em $\mathbb{R}^{n}$ e $c$, como foi dito, é uma constante real. A solução acima representa uma onda viajante $u(x, t)$ que viaja em $\mathbb{R}^{n}$ com velocidade constante $c$ no sentido do vetor $-\mathbf{n}$.

Fixando, a princípio, $t=t_{1}$ e $x \in \mathbb{R}^{n}$, obtemos o hiperplano $H_{1}: \mathbf{n} . x=d_{1}$, no qual a função $u$ é constante e igual a $u_{1}$. Para $t=t_{2}>t_{1}$, existe $d_{2} \in \mathbb{R}$ tal que a função $u$ é constante e igual a $u_{1}$ no hiperplano $H_{2}: \mathbf{n} . x=d_{2}$. Então, como $v\left(d_{1}+c t_{1}\right)=v\left(d_{2}+c t_{2}\right)$, temos $d_{1}+c t_{1}=d_{2}+c t_{2}$, uma vez que a existência e unicidade de soluções em problemas autônomos é garantida. E , portanto,

$$
d_{2}=d_{1}-c\left(t_{2}-t_{1}\right)
$$


Assim, observando que $d_{1}$ é a distância do hiperplano $\mathbf{n} \cdot x=d_{1}$ à origem, concluímos que a onda, de fato, viaja na direção $-\mathbf{n}$. Este é apenas um argumento geométrico mas que ilustra bem a dinâmica deste tipo de solução (ver 2.1).

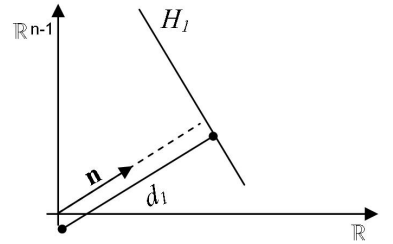

$t=t_{1}$

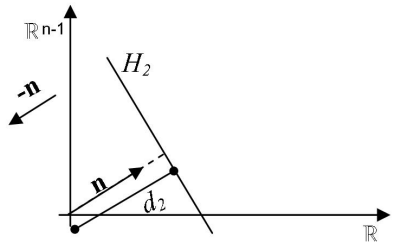

$t=t_{2}>t_{1}$

Figura 2.1: Dinâmica da solução $u=u_{1}$ para $t=t_{1}$ e $t=t_{2}>t_{1}$ fixo.

Ao contrário da equação da onda linear, que é hiperbólica e propaga qualquer padrão de onda com uma velocidade fixa, equações de reação-difusão permitem somente certos padrões de ondas se propagarem, cada uma com sua velocidade característica.

Dada a equação

$$
u_{t}=\Delta u+f(u)
$$

se procuramos por soluções do tipo ondas viajantes, a definição sugere alguns aspectos possíveis para $u$; por exemplo, a forma $u(x, t)=v(x-c t)$. Substituindo na equação acima, vemos que $v$ não pode ser uma função arbitrária, mas deve ser solução da seguinte EDO:

$$
v_{z z}+c v_{z}+f(v)=0
$$

que pode ser reduzida a um sistema de primeira ordem

$$
\begin{array}{r}
v_{z}=p \\
p_{z}=-c p+f(v) .
\end{array}
$$

Então, o estudo das soluções do problema 2.22 se reduz ao estudo das trajetórias - ou do retrato de fases - do sistema $2.24^{3}$.

Mostraremos agora, através de um exemplo, a importância do argumento acima no estudo das soluções de ondas em equações de reação-difusão. Iremos deduzir qualitativamente a existência e unicidade de ondas através de um retrato de fases construído como acima.

Exemplo 3 Considere a seguinte equação

$$
u_{t}=\Delta u+f(u)
$$

onde $f$ é uma função que tem como raízes pelo menos os pontos 0 , a e 1 , e cujo esboço é dado

\footnotetext{
${ }^{3}$ Ver, por exemplo, [16] e [36].
} 
pela figura 2.2. Além disso a é uma constante real pertencente a $(0,1)$. Procuramos por soluções da forma $u(x, t)=v(\boldsymbol{n} . x+c t)$, então, como foi feito acima, $v$ satisfaz a equação

$$
v_{z z}-c v_{z}+f(v)=0,
$$

e, portanto, o seguinte sistema

$$
\begin{array}{r}
v_{z}=p \\
p_{z}=c p-f(v) .
\end{array}
$$

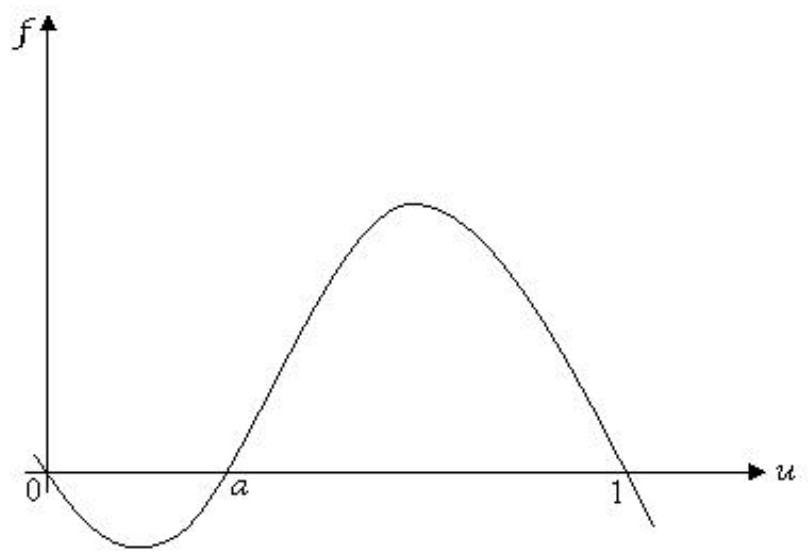

Figura 2.2: Esboço de $f$.

Observe que $u \equiv 0, u \equiv a$ e $u \equiv 1$ são pontos de equilíbrio da equação 2.26. Observamos ainda que o primeiro e o último são instáveis. Usando o mesmo argumento do exemplo anterior, obtemos um operador linear para $u \equiv 0$ e outro para $u \equiv 1, A_{0}$ e $A_{1}$ respectivamente, idênticos àqueles do mesmo exemplo.

Para que tenhamos de fato uma solução de onda limitada, iremos procurar por uma solução $v$ que conecte estes dois equilíbrios - de acordo com o argumento qualitativo feito na definição de ondas viajantes - para que, assim, um possa substituir o outro a medida em que a onda se propaga em $\mathbb{R}^{n}$. Isto implica que devemos ter

$$
v \rightarrow 0, \text { se } z \rightarrow-\infty \text { e } v \rightarrow 1 \text {, se } z \rightarrow \infty
$$

Vamos supor, a princípio, $c=0$. Então, uma integral primeira para o sistema

$$
\begin{array}{r}
v_{z}=p \\
p_{z}=-f(v) .
\end{array}
$$


é dada por

$$
E(p, v)=\frac{p^{2}}{2}+\int_{0}^{v} f(u) d u .
$$

De fato, ao longo das órbitas de 2.28 temos $E_{z}=p p_{z}+f(v) v_{z}=-p f(v)+f(v) p=0$, ou seja, $E$ é constante ao longo de cada órbita de 2.28, além disso, E não é constante em nenhum aberto de seu domínio.

Vamos agora esboçar o plano de fases $(v, p)$ e tentar encontrar $v$ que satisfaça as propriedades requeridas. Antes disso, faremos a seguinte hipótese: $\int_{0}^{1} f(u) d u>0$. Caso ela não seja satisfeita, basta fazer a mudança de coordenadas $u \longmapsto 1-u$. Observe que com esta hipótese obtemos

$$
\left|\int_{0}^{a} f(u) d u\right|<\int_{a}^{1} f(u) d u
$$

$e$, portanto, existe $b \in(a, 1)$ tal que $\int_{0}^{b} f(u) d u=0$. Observe também que, como as órbitas são dadas por $E(p, v)=\frac{p^{2}}{2}+\int_{0}^{v} f(u) d u=k$, com $k$ constante, então $p= \pm \sqrt{2 k-2 \int_{0}^{v} f(u) d u}$. O que significa que a representação das soluções é simétrica em relação ao eixo $v$.

Feitas estas observações podemos prosseguir com o propósito de obter o retrato de fases de 2.28. Vamos, a princípio, encontrar a órbita que passa pela origem de $(v, p)$. Ela é dada por $E=k_{1}$, onde $k_{1}=\frac{0^{2}}{2}+\int_{0}^{0} f(u) d u=0$. Então, a órbita que sai da origem cruza o eixo $v$ em $(b, 0)$, já que $\frac{0^{2}}{2}+\int_{0}^{b} f(u) d u=0$ e, devido a expressão obtida de $E=k$, isto acontece no sentido indicado na figura 2.3.

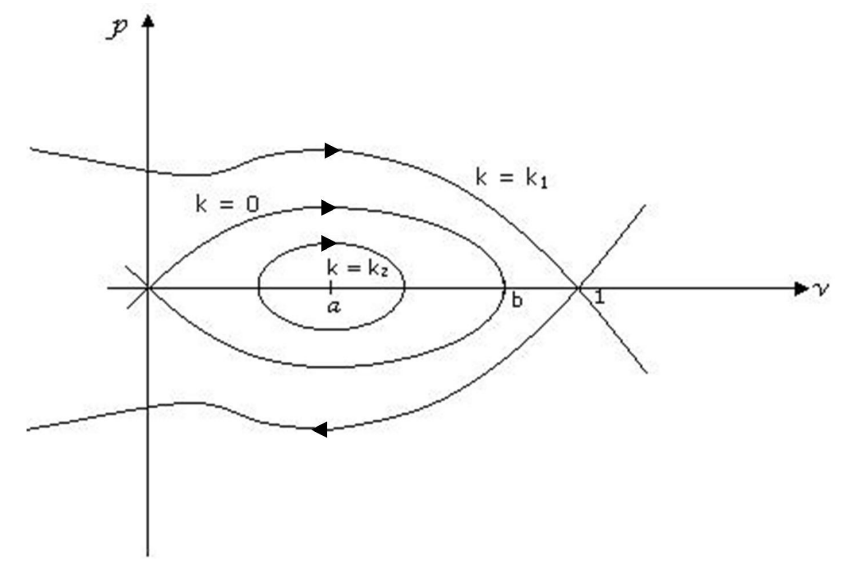

Figura 2.3: Retrato de fase para a equação 2.26 , caso $c=0$.

Vamos agora encontrar a órbita $E=k_{2}$ que passa por $(1,0)$. Devemos ter

$$
k_{2}=\frac{0^{2}}{2}+\int_{0}^{1} f(u) d u=\int_{0}^{1} f(u) d u>0
$$

além disso, esta mesma órbita cruza o eixo p em $\left(0, \sqrt{2 \int_{0}^{1} f(u) d u}\right)$ e é claro que $\sqrt{2 \int_{0}^{1} f(u) d u}>$ 0. É possivel obter ainda o seguinte fato: a órbita que passa por $(a, 0)$ é dada por $E=\int_{0}^{a} f(u) d u=$ 
$k_{0}$ e, na verdade, só contém este ponto.

Concluímos, dos argumentos acima, que a variedade instável de $(0,0), W^{u}(0,0)$, não contém o ponto $(1,0)$. Então, para $c=0$ não existe uma solução $v$ como a procurada. Partindo do fato de que as órbitas no plano de fases variam continuamente à medida que variamos c, tentaremos encontrar algum valor de c para o qual a variedade instável de $(0,0)$ coincida com a variedade estável de $(1,0)$, ou seja, $W^{u}(0,0)=W^{s}(1,0)$. Pois assim, teríamos

$$
\left(v, v_{z}\right) \rightarrow(0,0), \text { se } z \rightarrow-\infty \text { e }\left(v, v_{z}\right) \rightarrow(1,0), \text { se } z \rightarrow \infty
$$

e, portanto,

$$
v \rightarrow 0, \text { se } z \rightarrow-\infty \text { e } v \rightarrow 1, \text { se } z \rightarrow \infty
$$

como queríamos.

Considere um ponto $\left(v_{0}, p_{0}\right)$ qualquer no primeiro quadrante. Então a inclinação da órbita que passa por este ponto é dada por

$$
\frac{d p}{d v}\left(v_{0}, p_{0}\right)=\frac{c p_{0}-f\left(v_{0}\right)}{p_{0}}=c-\frac{f\left(v_{0}\right)}{p_{0}}
$$

o que significa que, à medida que c cresce, para cada ponto $v$, a inclinação das soluções, com relação ao mesmo eixo v, também deverá crescer. Um esboço deste fato é apresentado na figura 2.4. Isso acontece porque a inclinação das órbitas é uma função crescente em c. Então, conforme c cresce, a variedade instável $W^{u}(0,0)$ se move de modo tal que existirá um único valor de c, que denotaremos por $c_{0}$, para o qual ela contém o ponto $(1,0)$. Observe também que $k_{0}<k_{1}<k_{2}$, o que não contradiz o que foi dito anteriormente a respeito da relação entre a inclinação das órbitas e o valor de $c$.

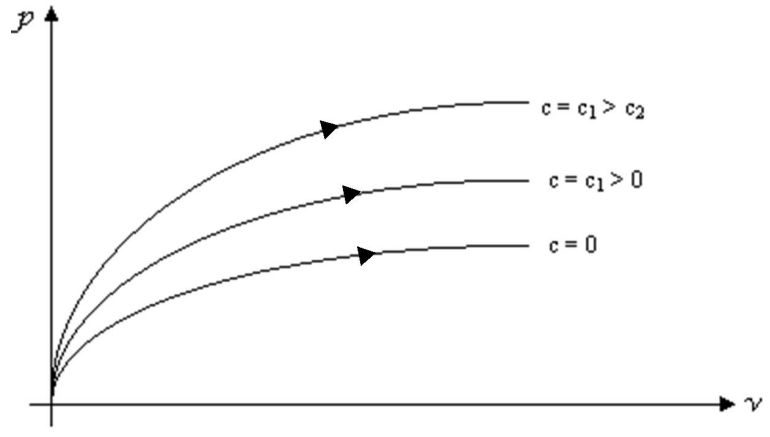

Figura 2.4: Esboço da variedade instável $W^{u}(0,0)$ para distintos valores de $c$.

Observamos inicialmente que $b=b(c)$ é uma função contínua e crescente de $c$. Além disso, existe um valor de $c$ - suponha $c=c_{0}$-, para o qual $b\left(c_{0}\right)>1$. Sendo assim, ou ainda, utilizando um argumento geométrico, análogo ao que foi feito acima, é possivel dizer que para valores de $c$ menores do que $c_{0}, W^{u}(0,0)$ cruza o eixo $v$ em algum ponto $\bar{v}<1$, ao passo que para valores de $c$ maiores do que $c_{0}, W^{u}(0,0)$ nunca cruzará o mesmo eixo. 
Deduzimos então a existência e unicidade de onda viajante, limitada pelos equilíbrios $u \equiv 0$ e $u \equiv 1$, como solução de 2.26 .

\subsubsection{Classificação e existência de ondas}

As soluções de ondas estudadas até agora eram de um particular tipo, conhecido como ondas planas - ou ondas em uma dimensão -, que são aquelas que se movem em $\mathbb{R}^{n}$ em uma direção fixa, com simetria por translação em qualquer direção perpendicular àquela do movimento. Este tipo de solução de onda é o mais simples e já foi amplamente estudado.

Ondas descritas por sistemas de reação-difusão se dividem em várias classes. Segue abaixo um breve comentário a este respeito.

Em geral as ondas se dividem em duas principais classes: a de ondas estácionárias e a de ondas periódicas, ainda que padrões distintos destes ainda sejam encontrados. Como exemplo podemos citar ondas do tipo espiral - ou rotating - e do tipo target.

As ondas estácionárias por sua vez, se dividem em ondas planas e ondas multidimensionais. Já as ondas periódicas se subdividem em ondas em uma dimensão, em duas dimensões, ondas do tipo spinning, ondas simétricas, ondas radiais e alguns outros tipos com estruturas mais complexas.

Não entraremos em detalhes a respeito das classes acima já que o foco deste trabalho recai em ondas planas, para o leitor interessado em uma classificação mais detalhada sugerimos [1]. Vamos nos restringir ao estudo deste particular tipo de ondas, que também se divide em alguns tipos, a saber:

(i) Wave fronts - são soluções $v(z)$ da equação 2.23 tais que existem os limites

$$
\lim _{z \rightarrow+\infty} v(z)=v_{+} e \lim _{z \rightarrow-\infty} v(z)=v_{-}
$$

e além disso, $v_{+} \neq v_{-}$. Como exemplo deste tipo de solução podemos citar aquela obtida no exemplo 2 acima - para a qual $v_{+}=1$ e $v_{-}=0$.

(ii) Pulses - são soluções de 2.23 tais que os limites em 2.29 existem mas $v_{+}=v_{-}$. Em geral estas soluções aparecem em equações de propagação de impulsos nervosos, conhecidas como equações de Hodgkin-Huxley e são da forma.

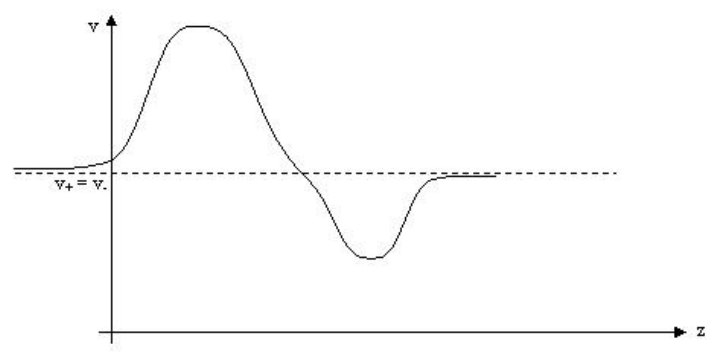

Figura 2.5: $v_{+}=v_{-}$.

(iii) Ondas periódicas no espaço - são soluções de 2.23 tais que $v(z)$ é uma função periódica. Tais soluções aparecem em problemas de cinética química e também equações de propagação de 
impulsos nervosos. Este tipo de solução corresponde a ciclos limites do retrato de fases obtidos do sistema 2.24 .

Para deduzir a existência - e unicidade - de soluções de ondas nos exemplos anteriores, reduzimos sistemas de segunda ordem a sistemas de EDO de primeira ordem e argumentamos de maneira qualitativa através de um retrato de fases. Isto foi possível pois tratavam-se de ondas em uma dimensão, para casos mais gerais existem outros tipo de métodos para provar a existência de ondas viajantes.

Diversos trabalhos se dedicaram ao estudo da existência de ondas em sistemas parabólicos e neles diferentes métodos foram empregados. Mas, correspondem basicamente a três tipos de abordagens.

Alguns têm como ferramenta métodos topológicos. Vamos citar, em particular, o método de Leray-Schauder que, grosseiramente falando, consiste na construção de uma deformação contínua ${ }^{4}$ de um dado sistema para outro cujas soluções existem e possuem propriedades requeridas.

Outro método amplamente usado - inclusive neste trabalho - é baseado no estudo de retrato de fases. Neste caso, a prova da existência de ondas se reduz à prova da existência de trajetórias correspondentes ao tipo de solução de ondas procurada. Por exemplo, para wave fronts ou pulses procuramos trajetórias que conectem os pontos $\left(v_{+}, 0\right)$ e $\left(v_{-}, 0\right)$, já ondas periódicas correspondem a ciclos limites.

O último método, que envolve teoria de bifurcações, é, em geral, utilizado para se detectar a existência de ondas viajantes. Mas pode também ser usado no caso de ondas multidimensionais.

A construção e o estudo de soluções de ondas para sistemas parabólicos não-lineares é uma área de grande interesse, não somente pelas aplicações das ondas em si, mas também por serem um instrumento importante para um melhor entendimento de fenômenos em domínios extensos, onde, pelo menos localmente, o comportamento se assemelha ao de ondas viajantes. Discutiremos na próxima subseção uma aplicação bastante consolidada na literatura que, ao passo que aborda um fenômeno relacionado a populações cíclicas, na seara da biologia, fornece um exemplo consistente do alcance da modelagem baseada nos termos discutidos até aqui.

\subsubsection{Ondas viajantes periódicas em populações cíclicas}

Ao longo da última década, diversos estudos de campo abordando populações com ciclos plurianuais detectaram ondas viajantes periódicas. Isto significa que os estudos indicaram que os ciclos diferem de fase em locais distintos de tal forma que um pico de densidade em um local ocorre simultaneamente com um poço em outro. Ou ainda, a densidade da população varia periodicamente em uma direção espacial, assim como em tempo, de forma que, quando combinadas, aparentam uma onda.

A velocidade desta 'onda' é dada pela razão entre os períodos em relação ao tempo e ao espaço. O período em $t$ é dado pelo número de anos de um ciclo completo de uma população em um ponto fixo do espaço, já o período em relação ao espaço $x$ é dado pelo comprimento de onda.

Existem diversos métodos de análise estatística para se detectarem ondas periódicas neste contexto. Um passo comum - e elementar - a eles consiste em estimativas do tamanho da população,

\footnotetext{
${ }^{4} \mathrm{Na}$ verdade, esta deformação é uma homotopia entre sistemas dinâmicos. Para maiores detalhes, ver [1].
} 
em diferentes pontos do espaço, ao longo do tempo. É também possível prever cenários nos quais ocorrem ondas periódicas e, inclusive, a relevância ecológica deste comportamento em populações animais. Entretanto, uma vez que o foco deste trabalho não reside sobre tais métodos, nem tampouco sobre seu desenvolvimento ou aplicação, indicaremos [1] ao leitor interessado e fecharemos este capítulo com a introdução do modelo sobre o qual este trabalho versará nas próximas páginas.

Considere duas populações cíclicas, com densidades $u$ e $v$. Um modelo de reação-difusão mais simples envolvendo-as é dado por

$$
\begin{gathered}
u_{t}=D_{1}\left(u_{x x}+u_{y y}\right)+f_{1}(u, v) \\
v_{t}=D_{2}\left(u_{x x}+u_{y y}\right)+f_{2}(u, v),
\end{gathered}
$$

onde $x$ e $y$ são coordenadas espaciais, $t$ denota tempo e $D_{1}, D_{2}$ são constantes. Além disso, devemos assumir que as dinâmicas locais de $f_{1}, f_{2}$ são tais que as equações

$$
u_{t}=f_{1}(u, v) \text { e } v_{t}=f_{2}(u, v)
$$

possuem uma solução periódica estável, que oscila de cada lado de um estado estacionário coexistente. Esta hipótese se deve ao fato de que o modelo se refere a densidade de populações cíclicas, mas isto ficará mais claro na última seção do capítulo ??, quando verificamos a existência de ondas viajantes periódicas para um particular modelo de reação-difusão.

Como fora dito no capítulo anterior, um de nossos principais objetivos é propor um teorema de existência e unicidade para 2.30 sujeito a condição inicial

$$
u(0)=u_{0}, \quad v(0)=v_{0},
$$

onde $u_{0}$ e $v_{0}$ são, a princípio, funções abritrárias definidas em $\mathbb{R}^{2}$, e estudar a estabilidade de possíveis soluções. Para tanto, alguns elementos da Análise Funcional, em particular da Teoria dos Operadores são necessários. Em função desta necessidade, o próximo capítulo versará sobre este tema, fornecendo os elementos para a discussão seguinte. 


\section{Capítulo 3}

\section{Teoria de semigrupos e o problema de Cauchy abstrato}

O objetivo deste capítulo é discutir a teoria de semigrupos de operadores lineares em espaços de Banach e suas aplicações à teoria das equações diferenciais ${ }^{1}$. Um dos pré-requisitos para o estudo deste tema envolve conceitos elementares da teoria espectral de operadores lineares. Neste sentido, introduzimos algumas definições e fatos a este respeito ${ }^{2}$.

\subsection{Teoria Espectral de Operadores Lineares}

\subsubsection{Conceitos e resultados básicos da teoria espectral}

Sejam $X \neq\{0\}$ um espaço normado complexo e $A: D(A) \subset X \longrightarrow X$ um operador linear com domínio $D(A)$. Associamos a cada $\lambda \in \mathbb{C}$ o operador

$$
A_{\lambda}=A-\lambda I
$$

onde $I$ é o operador identidade em $D(A)$. As propriedades do operador $A_{\lambda}$ dependem claramente de $\lambda$ e a teoria espectral versa basicamente sobre o estudo de tais propriedades. O operador inverso - ou, simplesmente inversa - de $A_{\lambda}$, que denotaremos por $R_{\lambda}$ e chamaremos de operador resolvente, pode ou não existir. Caso exista, o próximo passo é estudarmos sua continuidade e sobrejetividade. Neste sentido introduziremos algumas definições.

Definição 5 Seja $X$ um espaço vetorial normado e $D$ um subconjunto de $X$. Diremos que $D$ é denso em $X$ se para todo $x \in X$ e todo $\varepsilon>0$, a interseção

$$
D \bigcap \mathcal{B}_{\varepsilon}(x)
$$

for não vazia.

Definição 6 Sejam $X \neq 0$ um espaço normado complexo e $A: D(A) \subset X \longrightarrow X$ um operador linear com dominio $D(A)$. O conjunto

$$
\rho(A)=\left\{\lambda \in \mathbb{C} \mid R_{\lambda} \text { existe, é contínua e está definida em um subconjunto denso de } X\right\}
$$

\footnotetext{
${ }^{1}$ Sugerimos o clássico [29] ao leitor interessado em uma abordagem diferente envolvendo a teoria espectral dos operadores lineares e aplicações.

${ }^{2}$ Para mais detalhes acerca dos operadores lineares e algumas aplicações envolvendo semigrupos sugerimos [12].
} 
é chamado conjunto resolvente de $A$. Seu complementar no plano complexo $\mathbb{C}$, que denotaremos por $\sigma(A)$, é chamado espectro de A.

Definição 7 O conjunto dos autovalores, ou valores próprios, de A é dado por

$$
V P(A)=\left\{\lambda \in \mathbb{C} \mid \nexists R_{\lambda}\right\}
$$

Sendo $A$, como nas definições anteriores, um operador linear e contínuo, obtemos

$$
V P(A)=\{\lambda \in \mathbb{C} \mid N(A-\lambda I) \neq\{0\}\},
$$

onde $N(A-\lambda I)$ corresponde ao núcleo do operador $A-\lambda I$. Um ponto $x \in N(A-\lambda I) \backslash\{0\}$ é chamado autovetor - ou vetor-próprio - de $A$ associado ao autovalor $\lambda$. O subespaço de $D(A)$ formado pelos autovetores associados ao autovalor $\lambda$ e pelo vetor nulo de $X$ é chamado autoespaço de $A$ associado ao autovalor $\lambda$.

Dado este conjunto de definições, passaremos agora a uma discussão acerca das propriedades do conjunto espectral de $A$. Sendo $\sigma(A)$ o complementar de $\rho(A)$ em $\mathbb{C}$, ele é naturalmente dividido em três conjuntos disjuntos, a saber:

(i) o espectro pontual $\sigma_{p}(A)$ é conjunto $\operatorname{dos} \lambda^{\prime} s \in \sigma(A)$ tais que $R_{\lambda}$ não existe.

(ii) o espectro contínuo $\sigma_{c}(A)$ é conjunto $\operatorname{dos} \lambda^{\prime} s \in \sigma(A)$ tais que $R_{\lambda}$ existe mas não é contínuo.

(iii) espectro residual $\sigma_{r}(A)$ é conjunto dos $\lambda^{\prime} s \in \sigma(A)$ tais que $R_{\lambda}$ existe (contínua ou não) mas não está definida em um conjunto denso de $X$.

Em alguns casos nem todos os conjuntos acima são não-vazios. Por exemplo, se $\operatorname{dim} X<\infty$ temos $\sigma_{c}(A)=\sigma_{r}(A)=\emptyset$, pelo Teorema do Núcleo e Imagem ${ }^{3}$. Para tornar os conceitos acima um pouco mais claros apresentaremos um exemplo.

Exemplo 4 Considere o conjunto

$$
X=l^{2}(\mathbb{C})=\left\{\left.\left(x_{n}\right)_{n} \in \mathbb{C}^{\mathbb{N}}\left|\sum_{n \in \mathbb{C}}\right| x_{n}\right|^{2}<\infty\right\}
$$

com a norma $\left\|\left(x_{n}\right)_{n}\right\|_{l^{2}(\mathbb{C})}=\left(\sum_{n \in \mathbb{N}}\left|\left(x_{n}\right)\right|^{2}\right)^{\frac{1}{2}}$, e o operador $A: X \subset X \longrightarrow X$ dado por $\left(x_{n}\right)_{n} \longmapsto$ $\left(\frac{x_{n}}{n+1}\right)_{n}$. Então $0 \in \sigma_{c}(A)$. De fato, já que o núcleo de $N(A)$ só contém a seqüência identicamente nula, A é injetor e além disso sua imagem é densa em $X$ e $R(A) \neq X$, pois dada a seqüência $\left(\frac{1}{n+1}\right) \in X$ não existe nenhum $\left(x_{n}\right)_{n} \in X$ tal que $A\left(\left(x_{n}\right)_{n}\right)=\left(\frac{1}{n+1}\right)_{n}$.

Uma das propriedades do conjunto resolvente que será largamente utilizada neste trabalho é a igualdade

$$
\rho(-A)=-\rho(A)
$$

\footnotetext{
${ }^{3}$ Para uma exposição elementar do teorema do Núcleo e Imagem, remetemos o leitor ao texto de álgebra linear de Hoffman e Kunze [18]
} 
que pode ser obtida a partir de duas inclusões bastante simples a partir da definição de $\rho$.

Definição 8 Sejam A um conjunto e $\mathbb{K}$ o corpo escalar. Considere as operações

$$
\begin{aligned}
& +: A \times A \longrightarrow A, \\
& \bullet: A \times A \longrightarrow A
\end{aligned}
$$

$e$

$$
\cdot: \mathbb{K} \times A \longrightarrow A
$$

Se, para todo $x, y, z \in A$ e para todo $\alpha \in \mathbb{K}$

1. $(A,+, \cdot)$ é um espaço vetorial;

2. $x \bullet(y \bullet z)=(x \bullet y) \bullet z$;

3. $x \bullet(y+z)=(x \bullet y)+(x \bullet z) e(x+y) \bullet z=(x \bullet z)+(y \bullet z) e$

4. $\alpha \cdot(x \bullet y)=(\alpha \cdot x) \bullet y=x \bullet(\alpha \cdot y)$

diremos que $(A,+, \cdot)=\mathcal{A}$ é uma álgebra. Se for $\mathcal{A} \neq\{0\}$ e houver e $\in A$ tal que

$$
e \bullet x=x \bullet e=x
$$

para todo $x \in A$, diremos que A é uma álgebra com unidade. Mais ainda, se \|·\| é uma norma no espaço vetorial $(A,+, \cdot)$ tal que para todo $x$ y $\in$ A tem-se que

$$
\|x \bullet y\| \leq\|x\|\|y\|
$$

então $\mathcal{A}$ diz-se uma álgebra normada. Por fim, se A for um espaço completo sob a norma, dizemos que $\mathcal{A}$ é uma álgebra de Banach.

Lema 1 Seja $\mathcal{A}$ uma álgebra de Banach com unidade e. Então, para cada a $\in \mathcal{A}$ com $\|e-a\|<1$ existe $a^{-1} \in \mathcal{A}$. Além disso,

$$
a^{-1}=\sum_{n=0}^{\infty}(e-a)^{n}
$$

$e$

$$
\left\|a^{-1}-e\right\| \leq \frac{\|e-a\|}{1-\|e-a\|}
$$

Não apresentaremos a demonstração deste lema aqui. Indicamos ao leitor os livros de Megginson [32] e Kreyszig [30].

Consideramos no resultado seguinte $\mathcal{A}$ como sendo o conjunto dos operadores lineares e limitados $A: X \longrightarrow X$ equipado com a composição e funções como operação e cuja unidade coincide com o operador identidade no espaço de $\operatorname{Banach} X$. 
Corolário 1 Sejam $X$ um espaço de Banach e $A: X \longrightarrow X$ um operador linear e limitado tal que $\|I-A\|<1$. Então a inversa de A existe, é linear e limitada. Além disso,

$$
A^{-1}=\sum_{n=0}^{\infty}(I-A)^{n}
$$

e

$$
\left\|A^{-1}-I\right\| \leq \frac{\|I-A\|}{1-\|I-A\|}
$$

Teorema 2 Seja $X$ um espaço de Banach sobre $\mathbb{C}$ e $A: X \longrightarrow X$ um operador linear e limitado, então

$$
\{\lambda \in \mathbb{C}:|\lambda|>\|A\|\} \subset \rho(A)
$$

e

$$
(\lambda I-A)^{-1}=\sum_{n=0}^{\infty} \frac{A^{n}}{\lambda^{n+1}}
$$

para todo $\lambda \in \mathbb{C}$ com $|\lambda|>\|A\|$.

Demonstração. Seja $\lambda \in \mathbb{C}$ tal que $|\lambda|>\|A\|$. Definimos $B_{\lambda}=I-\frac{1}{\lambda} A$. É claro que $B_{\lambda}$ é um operador linear e limitado em $X$. De onde obtemos que

$$
\left\|I-B_{\lambda}\right\|=\left\|\frac{1}{\lambda} A\right\|=\frac{1}{|\lambda|}\|A\|<1 .
$$

Segue então, do corolário 1, que a inversa de $B_{\lambda}$ existe, é dada por

$$
B_{\lambda}^{-1}=\sum_{n=0}^{\infty}\left(I-B_{\lambda}\right)^{n}
$$

e é um operador linear e limitado. Escrevendo $\lambda I-A=\lambda\left(I-\frac{1}{\lambda} A\right)$, obtemos

$$
(\lambda I-A)^{-1}=\frac{1}{\lambda}\left(I-\frac{1}{\lambda} A\right)^{-1}=\frac{1}{\lambda} B_{\lambda}^{-1},
$$

que é também um operador linear e limitado. Portanto, $\lambda \in \rho(A)$ e

$$
(\lambda I-A)^{-1}=\frac{1}{\lambda} \sum_{n=0}^{\infty}\left(I-B_{\lambda}\right)^{n}=\frac{1}{\lambda} \sum_{n=0}^{\infty} \frac{A^{n}}{\lambda^{n}}=\sum_{n=0}^{\infty} \frac{A^{n}}{\lambda^{n+1}} .
$$

\subsubsection{Operadores fechados}

Estudaremos agora um particular tipo de operador linear, conhecido como operador fechado. Apresentaremos algumas definições e em seguida passaremos a alguns resultados. 


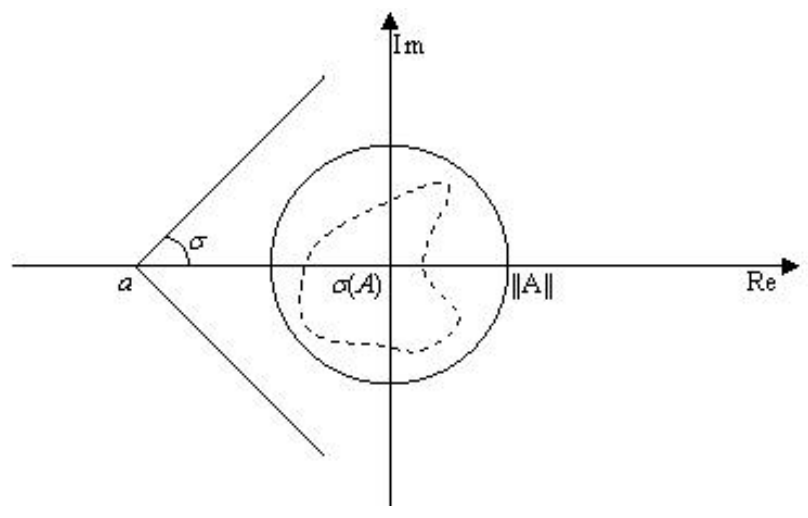

Figura 3.1: Ilustração de uma conseqüência da inclusão $\{\lambda \in \mathbb{C}: \lambda>\|A\|\} \subset \rho(A)$.

Definição 9 Sejam $X$ um espaço de Banach sobre um corpo $\mathbb{K}$ e $A: D(A) \longrightarrow X$, com $D(A) \subset$ $X$, um operador linear. Dizemos que A é fechado se seu gráfico

$$
G(A)=\{(x, y) \in X \times X ; x \in D(A) \text { e } y=A x\}
$$

for um subespaço fechado de $X \times X$.

Observe que, dizer que um operador linear $A$ é fechado, é equivalente a dizer que, dada qualquer seqüência $\left(x_{n}\right)$ em $D(A)$, com $x_{n} \rightarrow x$ e $A x_{n} \rightarrow y$, então $x \in D(A)$ e $y=A x$. De fato, se $A$ é fechado, dada qualquer seqüência $\left(x_{n}, A x_{n}\right)$ em $G(A) \operatorname{com}\left(x_{n}, A x_{n}\right) \rightarrow(x, y)$, então deve-se ter $(x, y) \in G(A)$, ou seja, $x \in D(A)$ e $y=A x$. A recíproca é óbvia. Note ainda que, o gráfico de um operador linear é sempre um subespaço, o que não é necessariamente verdade para o caso de uma aplicação qualquer - p.e., $(2,4)+(3,9)=(5,13)\left(5,5^{2}\right)$. Logo, o gráfico de $f(x)=x^{2}$, definida em $\mathbb{R}$, com valores em $\mathbb{R}$ não é um subspaço de $\mathbb{R} \times \mathbb{R}$.

Definição 10 Dado um espaço de Banach $X$ e denotando o fecho de $G(A)$ por $\overline{G(A)}$, um operador linear $A: D(A) \subset X \longrightarrow X$ é dito fechável se $(0, y) \in \overline{G(A)}$ implicar $y=0$.

Nas condições da definição acima, dizer que $A$ é fechável é equivalente a dizer que $\overline{G(A)}$ é o gráfico de um operador fechado. Neste caso, dizemos que este último operador é o fecho de $A$ e, em geral, ele é denotado por $\bar{A}^{5}$. A proposição que segue é mais uma equivalência da definição 9 e sua demonstração é obtida diretamente desta mesma definição.

Proposição 1 Dado um espaço de Banach $X$, então um operador linear $A: D(A) \subset X \longrightarrow X$ é fechável se, e somente se, para toda seqüência $x_{n} \in D(A)$, com $x_{n} \rightarrow 0$ e $A x_{n} \rightarrow y$, tivermos $y=0$.

Demonstração. Assumimos inicialmente que $A$ é um operador fechável. Consideramos então uma seqüência $x_{n} \in D(A)$ tal que $x_{n} \rightarrow 0$ e $A x_{n} \rightarrow y$, para algum $y \in Y$. Assim, obtemos que

\footnotetext{
${ }^{4}$ Em caráter de recordação, mencionamos que um espaço vetorial normado é dito um espaço de Banach se for completo sob sua norma, i.e., se toda sua seqüência de Cauchy for convergente.

${ }^{5}$ Não usaremos esta notação neste trabalho por uma questão de conveniência. No próximo capítulo estudaremos um operador $A$ que é, na verdade, o fecho do operador que chamaremos $B_{0} \times B_{0}$
} 
$\left(x_{n}, A x_{n}\right) \in G(A)$ e, como conseqüência, que $(0, y) \in \overline{G(A)}$, uma vez que $\left(x_{n}, A x_{n}\right) \rightarrow(0, y)$. De acordo com a definição de $\bar{A}$, temos ainda que $\overline{G(A)}=G(\bar{A})$ e, portanto, $0=\bar{A} 0=y$.

Reciprocamente, suponha que, para toda seqüência $x_{n} \in D(A)$, com $x_{n} \rightarrow 0$ e $A x_{n} \rightarrow y$, deve-se ter que $y=0$. Além disso, $\overline{G(A)}$ é um subspaço linear de $X \times Y$. Considerando algum elemento $(0, y) \in \overline{G(A)}$, temos que existe uma seqüência $x_{n} \in D(A)$ tal que $x_{n} \rightarrow 0$ e $A x_{n} \rightarrow y$. De onde obtemos, que $y=0$. Afirmamos então que para todo $x \in X$ deve existir no máximo um elemtento $y \in Y$ de modo que $(x, y) \in \overline{G(A)}$. De fato, suponha que existam $y, y^{\prime} \in Y$ tais que $(x, y),\left(x, y^{\prime}\right) \in \overline{G(A)}$. Sendo $\overline{G(A)}$ um subspaço, teríamos $\left(0, y-y^{\prime}\right) \in \overline{G(A)}$, o que seria um absurdo, já que $y-y^{\prime} \neq 0$. Podemos então definir o operador $\bar{A}$ como sendo $\bar{A} x=y$ onde $y$ é tal que $(x, y) \in \overline{G(A)}$. Segue que $\bar{A}$ é um operador linear e $\overline{G(A)}=G(\bar{A})$, uma vez que $\overline{G(A)}$ um subespaço linear.

Seguem uma proposição e dois lemas acerca das propriedades de um operador linear fechável.

Proposição 2 Sejam $X$ um espaço de Banach e $A: D(A) \subset X \longrightarrow X$ um operador linear injetor e $R(A)$ sua imagem. Então $A$ é fechável se, e somente se, $A^{-1}: R(A) \longrightarrow X$ é fechável.

Demonstração. Suponha que $A: D(A) \longrightarrow R(A)$ seja fechável. Para mostrar que $A^{-1}$ também é fechável, tomaremos uma seqüência $y_{n}$ em $R(A)$, com $y_{n} \rightarrow 0$ e $A_{n}^{-1} \rightarrow x$, e mostraremos que $x=0$. Como, para cada $n, y_{n} \in R(A)$ então existe $x_{n} \in D(A)$ tal que $x_{n}=A^{-1} y_{n}$. Considerando a seqüência $\left(x_{n}-x\right)_{n}$, temos

$$
x_{n}-x=A^{-1} y_{n}-x \rightarrow 0,
$$

então, como $A$ é fechável $A\left(x_{n}-x\right) \rightarrow 0$. Por outro lado,

$$
A\left(x_{n}-x\right)=A x_{n}-A x \rightarrow A x
$$

já que $A x_{n}=y_{n} \rightarrow 0$. Logo $A x=0$ e, sendo $A$ um operador linear e injetor, concluímos que $x=0$.

Lema 2 Dado um operador linear $A: D(A) \subset X \longrightarrow X$, se o conjunto resolvente $\rho(A)$ for não vazio, então A é fechável.

Demonstração. Sendo $\rho(A) \neq \emptyset$, existe algum $\lambda$ no corpo $\mathbb{K}$ tal que: $\lambda I-A$ é injetor, $\overline{R(\lambda I-A)}=$ $X$ e $(\lambda I-A)^{-1}$ é limitado. Mostraremos que $(\lambda I-A)^{-1}$ é fechável e, da proposição anterior, concluiremos que $(\lambda I-A)$ também é fechável. Com isso obtemos, diretamente da definição, que $A$ é fechável. A fim de provarmos que $(\lambda I-A)^{-1}: R(\lambda I-A) \longrightarrow X$ é fechável, tomaremos uma seqüência $x_{n}$ em $R(\lambda I-A)$, com $x_{n} \rightarrow 0$ e $(\lambda I-A)^{-1}\left(x_{n}\right) \rightarrow y$. Nosso objetivo é mostrar que $y=0$. De fato, sendo $(\lambda I-A)^{-1}$ um operador contínuo, $(\lambda I-A)^{-1}\left(x_{n}\right) \rightarrow 0$. Pela unicidade do limite, obtemos $y=0$.

Lema 3 Sejam $A_{0}: D\left(A_{0}\right) \subset X \longrightarrow X$ um operador linear e $A: D(A) \subset X \longrightarrow X$ o seu fecho. Se $A_{0}$ for fechável, então $\rho\left(A_{0}\right)=\rho(A)$. 
Demonstração. Considere inicialmente algum $\lambda$ arbitrário em $\rho(A)$, então $\lambda I-A$ é injetor, $\overline{R(\lambda I-A)}=X$ e $(\lambda I-A)^{-1}: R(\lambda I-A) \longrightarrow X$ é limitado. Sendo $A$ o fecho de $A_{0}$, valem as seguintes afirmações: $D\left(A_{0}\right) \subseteq D(A)$ e $A x=A_{0} x$, se $x \in D\left(A_{0}\right)$. De onde obtemos, juntamente com as hipóteses acima, que $\left(\lambda I-A_{0}\right)^{-1}: R\left(\lambda I-A_{0}\right) \longrightarrow X$ é limitado e $\left(\lambda I-A_{0}\right)$ é injetor. Resta mostrar que $\overline{R\left(\lambda I-A_{0}\right)}=X$. Então, considere um elemento $y$ em $X$ e $x=(\lambda I-A) y$. Obtemos, ainda pelo fato de $A$ ser o fecho de $A_{0}$, que existe uma seqüência $x_{n}$ em $D\left(A_{0}\right)$ com $x_{n} \rightarrow x$ e $(\lambda I-A) x_{n} \rightarrow y$. Portanto,

$$
y=\lim _{n \rightarrow \infty}\left(\lambda I-A_{0}\right) x_{n}, \text { onde }\left(\lambda I-A_{0}\right) x_{n} \in R(\lambda I-A),
$$

e concluímos que $\rho(A) \subseteq \rho\left(A_{0}\right)$.

Para provar que a inclusão contrária também é válida, tomaremos $\lambda \in \rho\left(A_{0}\right)$. Então $\lambda I-A_{0}$ é injetor, $\overline{R\left(\lambda I-A_{0}\right)}=X$ e $\left(\lambda I-A_{0}\right)^{-1}: R\left(\lambda I-A_{0}\right) \longrightarrow X$ é limitado. Seja $x \in D(A)$ tal que $(\lambda I-A) x=0$. Se provarmos que $x=0$, estará assim garantida a injetividade de $(\lambda I-A)$, já que este é um operador linear. Sabemos, pela definição do operador $A$, que existe uma seqüência $x_{n}$ em $D\left(A_{0}\right)$ tal que $x_{n} \rightarrow x$ e que $\left(\lambda I-A_{0}\right) x_{n} \rightarrow 0$. Sendo $\left(\lambda I-A_{0}\right)^{-1}$ um operador contínuo, temos que

$$
\left(\lambda I-A_{0}\right)^{-1}\left(\lambda I-A_{0}\right) x_{n} \rightarrow 0
$$

Portanto, $x=0$. A afirmação de que $R(\lambda I-A)$ é denso em $X$, segue diretamente dos seguintes fatos: $R(\lambda I-A)$ é um conjunto fechado em $X$ - pela definição do operador $A-, R\left(\lambda I-A_{0}\right) \subseteq R(\lambda I-A)$ e $\overline{R\left(\lambda I-A_{0}\right)}=X$. Finalmente, dado $y \in R(\lambda I-A)$, mostraremos $(\lambda I-A)$ é contínuo em $y$. Sabemos que existe uma seqüência $y_{n}$ em $R\left(\lambda I-A_{0}\right)$ tal que $y_{n} \rightarrow y$ e $\left(\lambda I-A_{0}\right)^{-1} y_{n} \rightarrow(\lambda I-A)^{-1} y$. Segue que

$$
\left\|(\lambda I-A)^{-1} y\right\|=\left\|\lim _{n \rightarrow \infty}\left(\lambda I-A_{0}\right)^{-1} y_{n}\right\| \leq \lim _{n \rightarrow \infty}\left\|\left(\lambda I-A_{0}\right)^{-1}\right\|\left\|y_{n}\right\|=M\|y\|,
$$

onde $M=\left\|\left(\lambda I-A_{0}\right)^{-1}\right\|$. Observe que usamos o fato de que a norma e $\left(\lambda I-A_{0}\right)^{-1}$ são funções contínuas. Concluímos então que $\lambda \in \rho(A)$, de onde segue o resultado.

\subsubsection{Operadores adjuntos, simétricos e auto-adjuntos}

Sejam $X$ e $Y$ espaços normados. Denotaremos por $\mathcal{L}(X, Y)$ o conjunto dos operadores lineares e contínuos $A: X \longleftarrow Y$ e por $X^{*}$ o conjunto dos funcionais lineares $\phi: X \longrightarrow \mathbb{K}$, onde $\mathbb{K}$ é um corpo, ou seja, o espaço dual de $X$. Dado $x^{*} \in X^{*}$, denotaremos o seu valor em um vetor $x \in X$, ou seja, a sua ação sobre o ponto $x$, por $\left\langle x^{*}, x\right\rangle$ ou $\left\langle x, x^{*}\right\rangle$.

Definição 11 Sejam $X$ um espaço de Banach sobre um corpo $\mathbb{K}, X^{*}$ o seu dual e $A: D(A) \subset$ $X \longrightarrow X$ um operador linear com domínio $D(A)$ denso em $X$. O adjunto de $A$ é um operador

$$
A^{*}: D\left(A^{*}\right) \subset X^{*} \longrightarrow X^{*}
$$


definido da seguinte forma:

$$
D\left(A^{*}\right)=\left\{x^{*} \in X^{*} ; \exists y^{*} \in X^{*} \operatorname{com}<x^{*}, A x>=<y^{*}, x>\quad \forall x \in D(A)\right\}
$$

e, dado $x^{*} \in D\left(A^{*}\right)$, definimos $A^{*} x^{*}=y^{*}$, onde $y^{*}$ é o elemento de $X^{*}$ que satisfaz 3.6.

Observe que o operador $A^{*}$ está bem definido pois, como $D(A)$ é denso em $X$, existe no máximo um elemento $y^{*} \in X^{*}$ satisfazendo 3.6.

Proposição 3 Sejam $X$ um espaço de Banach, $X^{*}$ o seu dual e $A: D(A) \subset X \longrightarrow X$ um operador linear com domínio $D(A)$ denso em $X$. Então, o adjunto de $A$,

$$
A^{*}: D\left(A^{*}\right) \subset X^{*} \longrightarrow X^{*}
$$

é um operador fechado, ou seja, o gráfico $G\left(A^{*}\right)$ é fechado em $X^{*} \times X^{*}$.

Demonstração. Seja $\left(x_{n}^{*}\right)_{n}$ uma seqüência em $D\left(A^{*}\right)$ tal que $x_{n}^{*} \rightarrow x^{*}$ e $A^{*} x_{n}^{*} \rightarrow y^{*}$, ou seja, a seqüência $\left(x_{n}^{*}, A^{*} x_{n}^{*}\right)$ em $G\left(A^{*}\right)$ converge para $\left(x^{*}, y^{*}\right)$. Nosso objetivo é provar que $\left(x^{*}, y^{*}\right) \in$ $G\left(A^{*}\right)$, para isso devemos ter $y^{*}=A^{*}\left(x^{*}\right)$. De fato, temos

$$
<x^{*}, A x>=<\lim _{n} x_{n}^{*}, A x>=\lim _{n}<x_{n}^{*}, A x>=\lim _{n}<A^{*} x_{n}^{*}, x>=<\lim A^{*} x_{n}^{*}, x>=<y^{*}, x>.
$$

O que implica, pela definição do operador adjunto, que $y^{*}=A^{*}\left(x^{*}\right)$.

Definição 12 Seja $H$ um espaço vetorial normado, cuja norma provém de um produto interno, i.e., para todo $x \in H$,

$$
\|x\|=\sqrt{\langle x, x\rangle}
$$

Se $H$ for completo, sob esta norma, diremos que $H$ é um espaço de Hilbert.

Sejam $H$ um espaço de Hilbert com produto interno $\langle\cdot, \cdot\rangle$ e $A: D(A) \subset X \longrightarrow X$ um operador linear com $\overline{D(A)}=H$. Afim de considerarmos o operador $A^{*}$ como um operador em $\mathrm{H}$, faremos a seguinte identificação

$$
H^{*} \cong H,
$$

e, denotaremos ambos por $H$. Isto é possível, e na verdade usual, pois pelo teorema da Representação de Riesz para funcionais, cada funcional linear limitado definido sobre um espaço de Hilbert pode ser identificado de maneira unívoca com um ponto do espaço. Para detalhes, ver [30].

Definição 13 Nas condições acima, dizemos que $A$ é um operador simétrico se $A \subset A^{*}$, ou seja, se

$$
<A x, y>=<x, A y>, \quad \forall x, y \in D(A) .
$$

Dizemos que A é auto-adjunto se

$$
A=A^{*},
$$


ou, mais precisamente, se $D(A)=D\left(A^{*}\right)$.

Teorema 3 Sejam $H$ um espaço de Hilbert e $A: D(A) \subset H \longrightarrow H$ um operador auto-adjunto tal que

$$
\langle A x, x\rangle \geq m\|x\|^{2}
$$

para todo $x \in D(A)$ então $\sigma(A) \subset[m, \infty)$.

Demonstração. Demonstraremos que, para um operador linear auto-adjunto $A$ entre espaços de Hilbert complexos, seu espectro está contido no intervalo $[m, M]$, onde

$$
m=\inf _{\|x\|=1}\langle A x, x\rangle
$$

e

$$
M=\sup _{\|x\|=1}\langle A x, x\rangle .
$$

Assim, para concluirmos o resultado acima basta apenas notarmos que no nosso caso $M=+\infty$.

Sabe-se que o espectro de $A$ é um subconjunto da reta real. Seja $\lambda_{\varepsilon}=M+\varepsilon \operatorname{com} \varepsilon>0$. Mostraremos que para qualquer $\varepsilon>0$ tem-se que $\lambda_{\varepsilon} \in \rho(A)$. Tomemos então $x \neq 0$ e $v=\|x\|^{-1} x$. Temos então que $x=\|x\| v \mathrm{e}$

$$
\langle A x, x\rangle=\|x\|^{2}\langle A v, v\rangle \leq\|x\|^{2} \sup _{\|v\|=1}\langle A v, v\rangle=\langle x, x\rangle M
$$

Assim, $-\langle A x, x\rangle \geq-\langle x, x\rangle M$ e, utilizando a desigualdade de Cauchy-Schwatrz, obtem-se:

$$
\left\|\left(\lambda_{\varepsilon} I-A\right) x\right\| \geq \varepsilon\|x\|^{2},
$$

$\operatorname{com} \varepsilon=\lambda_{\varepsilon}-M>0$. Logo, obtemos que

$$
\left\|\left(\lambda_{\varepsilon} I-A\right) x\right\| \geq \varepsilon\|x\|,
$$

o que implica diretamente que $\lambda_{\varepsilon} \in \rho(A)$. De maneira análoga, obtemos que qualquer $\lambda_{\delta}=m-\delta$, com $\delta>0$ pertence a $\rho(A)$ e, assim, segue o resultado.

Para concluirmos esta subseção, vamos mencionar dois resultados acerca dos operadores simétricos - em sua relação com os operadores auto-adjuntos - que têm como propósito ilustrar em algum sentido a maneira como estas classes de operadores se relacionam.

Lema 4 Seja $A$ um operador simétrico e, para algum $\lambda \in \mathbb{C}$

$$
R(\lambda I-A)=H \quad \text { e } \overline{R(\lambda I-A)}=H,
$$

então $A$ é auto-adjunto ${ }^{6}$.

\footnotetext{
${ }^{6}$ Ver $[9]$
} 
Lema 5 Se $A$ for um operador simétrico e $R(A)=H$, então A é auto-adjunto.

Demonstração. Imediato a partir do lema 4.

\subsubsection{Operadores dissipativos}

Na próxima seção discutiremos a teoria de semigrupos de operadores lineares, que são unicamente determinados pelos seus geradores. Uma importante caracterização de tais geradores envolve o conceito de operadores dissipativos. Dedicaremos esta subseção ao estudo destes operadores.

Definição 14 Seja $X$ um espaço de Banach sobre um corpo $\mathbb{K}$ e $X^{*}$ seu dual. Dizemos que a correspondência, ou função multívoca, $J: X \longrightarrow 2^{X^{*}}$ dada por

$$
J(x)=\left\{x^{*} \in X^{*}: \operatorname{Re}\left\langle x^{*}, x\right\rangle=\|x\|^{2}=\left\|x^{*}\right\|^{2}\right\}
$$

é a aplicação dualidade de $X$, onde $2^{X^{*}}$ denota o conjunto das partes de $X^{*}$.

Observe que, para cada $x \in X$, o conjunto $J(x)$, que chamaremos de conjunto dualidade de $x$, é não-vazio como conquência do teorema de Hahn-Banach. ${ }^{7}$

Definição 15 Seja $X$ um espaço de Banach sobre um corpo K. Dizemos que um operador linear $A: D(A) \subset X \longrightarrow X$ é dissipativo se

$$
D(A) \subset\left\{x \in X: \exists x^{*} \in J(x) \operatorname{com} \operatorname{Re}\left\langle x^{*}, A x\right\rangle \leq 0\right\}
$$

Um operador linear $B: D(B) \subset X \longrightarrow X$ é dito acretivo ${ }^{8}$, ou maximal monótono, se $A=-B$ for um operador dissipativo.

Segue um exemplo de operador dissipativo bastante simples, mas que ilustra apropriadamente o caso em que $X$ tem dimensão finita.

Exemplo 5 Sejam $X=\mathbb{R}^{n}$ e $A: X \rightarrow X$ o operador negativo da identidade em $X$, isto é, $A x=-x$ para todo $x \in X$. Afirmamos que $A$ é dissipativo. De fato, sendo $X$ um espaço de Hilbert, podemos identificar cada elemento $x \in X$ com o funcional linear $x^{*}$ dado por

$$
\left\langle x^{*}, y\right\rangle=\langle y, x\rangle, \quad \forall y \in \mathbb{R}^{n}
$$

Calculemos agora a norma do operador $x^{*}$ :

$$
\left\|x^{*}\right\|=\sup _{y \in B_{X}}\left|\left\langle x^{*}, y\right\rangle\right|=\sup _{y \in B_{X}}|\langle x, y\rangle| \leq \sup _{y \in B_{X}}\|x\|\|y\| \leq\|x\| .
$$

\footnotetext{
${ }^{7} \mathrm{O}$ teorema de Hanhn-Banach, e suas conseqüências, têm demasiada importância no contexto da Análise e de suas aplicações. Por esta razão, remetemos o leitor ao texto de Robert Megginson, [32].

${ }^{8}$ Ver $[20]$
} 
Em particular, $\frac{x}{\|x\|} \in B_{X}$ e

$$
\left|\left\langle x, \frac{x}{\|x\|}\right\rangle\right|=\|x\| .
$$

Portanto, para cada $x \in X$, o funcional $x^{*} \in J(x)$. Segue então que, para cada $x \in X$, existe $x^{*} \in J(x)$ - isto se deve ao fato de que uma das propriedades deste operador nos garante que $\left\|x^{*}\right\|=\|x\|-$ tal que

$$
R e\left\langle x^{*}, A x\right\rangle=\langle A x, x\rangle=-\langle x, x\rangle=-\|x\|^{2} \leq 0 .
$$

De onde segue que A um operador dissipativo.

O objetivo dos próximos resultados é apresentar algumas propriedades de operadores dissipativos. O principal deles, o teorema de G.Lumer, mostra que se $A$ é dissipativo então podemos limitar o operador resolvente de $A$.

Lema 6 Sejam $X$ um espaço de Banach e $A: D(A) \subset X \longrightarrow X$ operador linear. Então, $A$ é dissipativo se, e somente se,

$$
\|(\lambda-A) x\| \geq \lambda\|x\|, \quad \forall x \in D(A), \quad \forall \lambda>0 .
$$

Demonstração. Suponha que $A$ seja dissipativo e escolha arbitrariamente $\lambda>0$ e $x \in D(A)$. Sabemos então que existe $x^{*} \in J(x)$ tal que $\operatorname{Re}\left\langle A x, x^{*}\right\rangle \leq 0$. Segue que

$$
\|\lambda x-A x\|\|x\|=\|\lambda x-A x\|\left\|x^{*}\right\| \geq\left|\left\langle\lambda x-A x, x^{*}\right\rangle\right| \geq \operatorname{Re}\left\langle\lambda x-A x, x^{*}\right\rangle=\lambda \operatorname{Re}\left\langle x, x^{*}\right\rangle=\lambda\|x\|^{2} .
$$

Observe que na primeira desigualdade usamos Cauchy-Schwartz. Segue então o resultado para o caso $x \neq 0$. O outro caso é óbvio.

Reciprocamente, considere algum $x \in D(A)$. Queremos provar que existe $x^{*} \in J(x)$ tal que $\operatorname{Re}\left\langle x^{*}, A x\right\rangle \leq 0$. Tomando $y_{\lambda}=\lambda x-A x$ sabemos, pela definição do conjunto dualidade, que existe algum $y_{\lambda}^{*} \in J\left(y_{\lambda}\right)$. Definindo $z_{\lambda}^{*}=\frac{y_{\lambda}^{*}}{\left\|y_{\lambda}^{*}\right\|}$ e, é claro, supondo que a afirmação 3.7 é válida, obtemos

$$
\lambda\|x\| \leq\left\|y_{\lambda}\right\|=\operatorname{Re}\left\langle y_{\lambda}, y_{\lambda}^{*}\right\rangle \frac{1}{\left\|y_{\lambda}^{*}\right\|}=\operatorname{Re}\left\langle y_{\lambda}, z_{\lambda}^{*}\right\rangle=\lambda \operatorname{Re}\left\langle x, z_{\lambda}^{*}\right\rangle-\operatorname{Re}\left\langle A x, z_{\lambda}^{*}\right\rangle \leq \lambda\|x\|-\operatorname{Re}\left\langle A x, z_{\lambda}^{*}\right\rangle .
$$

Segue que $\operatorname{Re}\left\langle A x, z_{\lambda}^{*}\right\rangle \leq 0$ para todo $\lambda>0$, já que este foi tomado arbitrariamente. Um resultado bastante conhecido e importante da Análise Funcional diz que, na topologia fraca*, a bola unitária de qualquer espaço de Banach é compacta. Então, como $X^{*}$ é um espaço de Banach e $z_{\lambda}^{*} \in B_{X^{*}}$, existem uma seqüência $\lambda_{n} \rightarrow \infty$ e algum $z^{*} \in B_{X^{*}}$ tais que $g_{\lambda_{n}}^{*} \rightarrow z^{*}$, na topologia fraca*.

Da desigualdade acima, temos que $\operatorname{Re}\left\langle A x, z^{*}\right\rangle \leq 0$ e $\operatorname{Re}\left\langle x, z^{*}\right\rangle \geq\|x\|$. Como $\operatorname{Re}\left\langle x, z^{*}\right\rangle \leq$ $\left|\left\langle x, z^{*}\right\rangle\right| \leq\|x\|$, obtemos que

$$
\left\langle x, z^{*}\right\rangle=\|x\| .
$$

Tomando $x^{*}=\|x\| z^{*}$ segue que $x^{*} \in J(x)$ e $\operatorname{Re}\left\langle A x, x^{*}\right\rangle \leq 0$. Assim, concluímos que $A$ é dissipativo. 
Definição 16 Sejam $T: D(T) \subset X \longrightarrow Y$ um operador linear, com $X$ e $Y$ espaços vetoriais normados. Então, diremos que $T$ é densamente definido em $X$ se $D(T)$ for um subconjunto denso de $X$.

Lema 7 Se A é dissipativo e densamente definido então A é fechável. Além disso, seu fecho $\bar{A}$ também é um operador dissipativo.

Demonstração. Suponha que $A: D(A) \subset X \longrightarrow X$ seja dissipativo e $\overline{D(A)}=X$ mas que $A$ não seja fechável. Então, existe uma seqüência $\left(x_{n}\right)$ em $X$ tal que $x_{n} \rightarrow 0$ e $A x_{n} \rightarrow y$, com $\|y\|=1$. Na verdade, a proposição 1 nos garante apenas que existe $\|y\| \neq 0$, mas basta então tomar a seqüência $\left(\frac{x_{n}}{\|y\|}\right)$, que também converge para 0 e $A \frac{x_{n}}{\|y\|}$ converge para $\frac{y}{\|y\|}$, que tem norma 1.

Obtemos assim, do lema 6, que

$$
\left\|\left(x+t^{-1} x_{n}\right)-t A\left(x+t^{-1} x_{n}\right)\right\| \geq\left\|x+t^{-1} x_{n}\right\|
$$

para quaisquer $t>0$ e $x \in D(A)$. Tomando o limite quando $n \rightarrow \infty$ e, em seguida, o limite quando $t \rightarrow 0$, concluímos que

$$
\|x-y\| \geq\|x\|
$$

para todo $x \in D(A)$. O que seria um absurdo pois $D(A)$ é denso em $X$. Logo $A$ é um operador fechável.

Afirmamos ainda que o operador $\bar{A}$ é dissipativo. De fato, sejam $x \in D(\bar{A})$ e $y=\bar{A} x$. Existe então uma seqüência $\left\{x_{n}\right\}$ em $D(A)$ tal que $x_{n} \rightarrow x$ e $A x_{n} \rightarrow y=\bar{A} x$. Obtemos, do lema 6 , que

$$
\left\|\lambda x_{n}-A x_{n}\right\| \geq \lambda\left\|x_{n}\right\|
$$

para todo $\lambda>0$. Tomando o limite quando $n \rightarrow \infty$ obtemos ainda que

$$
\|\lambda x-\bar{A} x\| \geq \lambda\|x\|
$$

para todo $\lambda>0$. Como $x \in D(\bar{A})$ é arbitrário, concluímos, pelo lema 6 , que o operador $\bar{A}$ é dissipativo.

Teorema 4 [G.Lumer] Se $A: D(A) \subset X \longrightarrow X$ é um operador dissipativo tal que $\overline{D(A)}=X$ e, para algum $\lambda_{0}>0, \lambda_{0}-A$ é sobrejetor então $(0, \infty) \subset \rho(A) e$

$$
\left\|(\lambda I-A)^{-1}\right\| \leq \frac{1}{\lambda}, \quad \forall \lambda>0 .
$$

Demonstração. Tomemos $\lambda>0$ e um ponto no domínio de $A$, digamos $x \in D(A)$. Segue do lema 6 que

$$
\|(\lambda I-A) x\| \geq \lambda\|x\|
$$

Assim, além de sabermos que $R\left(\lambda_{0} I-A\right)=X$, temos que $\left\|\left(\lambda_{0} I-A\right) x\right\| \geq \lambda_{0}\|x\|$ para todo $x \in D(A)$. Assim, $\lambda_{0}$ pertence ao conjunto resolvente do operador fechado $A$. Como $\mathbb{R}^{+}$é conexo, 
então um seu subconjunto $\Lambda$ será aberto e fechado em $\mathbb{R}^{+}$se e somente se for $\Lambda=\mathbb{R}^{+}$.

Seja $\Lambda=\rho(A) \cap \mathbb{R}^{+}$. Do fato de $\rho(A)$ ser aberto, segue que $\Lambda$ é aberto. Queremos ver que $\Lambda$ é ainda fechado em $\mathbb{R}^{+}$, o que leva imediatamente a $\rho(A) \supset \mathbb{R}^{+}$. Tomemos uma seqüência $\left(\lambda_{n}\right)_{n \in \mathbb{N}}$ de elementos de $\Lambda \operatorname{com} \lambda_{n} \rightarrow \lambda$. Existe $n^{*} \in \mathbb{N}$ tal que se $n>n^{*}$, então

$$
\left|\lambda_{n}-\lambda\right|<\frac{\lambda}{3}
$$

Assim, para valores grandes de $n$ temos

$$
\left\|\left(\lambda-\lambda_{n}\right)\left(\lambda_{n}-A\right)^{-1}\right\| \leq\left|\lambda_{n}-\lambda\right| \lambda_{n}^{-1} \leq \frac{1}{2}
$$

$\mathrm{e}$

$$
I+\left(\lambda-\lambda_{n}\right)\left(\lambda_{n}-A\right)^{-1}
$$

é um isomorfismo de X. Então a aplicação $\lambda-A$ aplica $D(A)$ sobrejetivamente em $X$, ao passo que $\lambda \in \rho(A)$, o que implica diretamente no resultado.

\section{$3.2 \quad$ Semigrupos de operadores lineares}

Com intuito de estudar as técnicas de solução de problemas parabólicos, na verdade de um particular problema de reação-difusão, estudaremos nesta seção fatos da teoria de semigrupos lineares e contínuos.

\subsubsection{Definições e resultados básicos}

Seja $X$ um espaço de Banach. O conjunto $\mathcal{L}(X)$, como foi dito, denota o espaço dos operadores lineares e contínuos de $X$ em $X$. Consideraremos este conjunto com a seguinte norma

$$
\|T\|_{L(X)}=\sup _{x \in B_{X}}\|T x\|_{X},
$$

onde $B_{X}=\left\{x \in X ;\|x\|_{X}=1\right\}$.

Definição 17 Seja $X$ um espaço de Banach. Uma família a um parâmetro $\{T(t): t \geq 0\} \subset \mathcal{L}(X)$ é dita semigrupo de operadores lineares em $X$ se as seguintes condições forem satisfeitas:

1. $T(0)=I_{X}$, onde $I_{X}$ é a função identidade de $X$ em $X$;

2. $T(t+s)=T(t) T(s)$, para todo $t, s \geq 0$.

Se além disso,

3. $\lim _{t \rightarrow 0^{+}}\left\|T(t)-I_{X}\right\|=0$, dizemos que o semigrupo é uniformemente contínuo (em $\left.t=0\right)$.

4. $\lim _{t \rightarrow 0^{+}} T(t) x=x$, para todo $x \in X$, dizemos que o semigrupo é fortemente contínuo.

Todo semigrupo fortemente contínuo possui uma limitação exponencial que é dada pelo seguinte teorema. 
Teorema 5 Seja $\{T(t): t \geq 0\} \subset \mathcal{L}(X)$ um semigrupo fortemente contínuo. Então existem constantes $\beta \geq 0$ e $M \geq 1$ tais que

$$
\|T(t)\| \leq M e^{\beta t}, \quad \forall t \geq 0
$$

Demonstração. Limitaremos inicialmente $\|T(t)\|$ em um intervalo da forma $[0, \eta]$ e depois estenderemos por todo o semi-eixo positivo. Suponha, por contradição, que $\eta>0$ tal que $\|T(t)\|$ seja limitado em $[0, \eta]$. Então existiria uma seqüência $\left(t_{n}\right)_{n}$ em $(0, \infty)$ tal que $t_{n} \rightarrow 0^{+}$e $\left\|T\left(t_{n}\right)\right\| \geq n$. Assim, pelo Princípio da Limitação Uniforme, existiria algum $x \in X$ tal que a seqüência $\left(\left\|T\left(t_{n}\right) x\right\|\right)_{n}$ seria ilimitada. O que geraria uma contradição, já que $\lim _{t \rightarrow 0^{+}} T(t) x=x$, para todo $x$ em $X$. Portanto, existe uma constante $M$ tal que $\|T(t)\| \leq M, \quad \forall t \in[0, \eta]$. Observe que $M \geq 1$, já que $\|T(0)\|=\left\|I_{X}\right\|=1$. Dado qualquer $t \in(0, \infty)$, podemos escreve-lo na forma $t=n \eta+\delta$ com $0 \leq \delta<\eta$. Segue das propriedades de semigrupos que

$$
\|T(t)\|=\|T(n \eta) T(\delta)\| \leq\|T(\eta)\|^{n}\|T(\delta)\| \leq M^{n} M \leq M M^{\frac{t}{\eta}}=M e^{\beta t},
$$

onde $\beta=\eta^{-1} \ln M \geq 0$. Usamos na desigualdade acima o seguinte fato

$$
\frac{t}{\eta}=n+\frac{\delta}{\eta} \Rightarrow \frac{t}{\eta} \geq n \Rightarrow M^{n} \leq M^{\frac{t}{\eta}}
$$

Concluímos então que $\|T(t)\| \leq M e^{\beta t}, \quad \forall t \in(0, \infty)$.

Corolário 2 Seja $\{T(t): t \geq 0\} \subset \mathcal{L}(X)$ um semigrupo fortemente contínuo. Então, para todo $x \in X$, a função $T() x:. \mathbb{R}^{+} \longrightarrow X$, dada por $t \longmapsto T(t) x$ é contínua.

Demonstração. Queremos provar que, para cada $x \in X$, vale que

$$
\lim _{h \rightarrow 0^{+}} T(t+h) x=T(t) x=\lim _{h \rightarrow 0^{-}} T(t+h) x .
$$

Observe que a segunda igualdade é equivalente a $\lim _{h \rightarrow 0^{+}} T(t-h) x=T(t) x$. Então, seja $h \geq 0$. Segue, pelo teorema anterior e pelas propriedades de semigrupo, que

$$
\|T(t+h) x-T(t) x\| \leq\|T(t)\|\|T(h) x-x\| \leq M e^{\beta t}\|T(h) x-x\|
$$

e

$$
\|T(t-h) x-T(t) x\| \leq\|T(t-h)\|\|T(h) x-x\| \leq M e^{\beta t}\|T(h) x-x\| .
$$

Tomando o limite em 3.8 e 3.9 , quando $h \rightarrow 0^{+}$, e usando o fato de $T(t)^{9}$ ser um semigrupo fortemente contínuo, obtemos o resultado desejado.

Dado um semigrupo fortemente contínuo de operadores lineares, podemos associá-lo a um operador linear $A$ - através da definição seguinte - e por conseqüência também associá-lo a uma equação

\footnotetext{
${ }^{9}$ Ao longo deste texto nos referimos ao semigrupo $\{T(t): t \geq 0\}$ como semigrupo $T(t)$.
} 
diferencial da forma

$$
\dot{u}=A u \text {. }
$$

Veremos mais a frente a importância desta identificação.

Definição 18 Seja $\{T(t): t \geq 0\} \subset \mathcal{L}(X)$ um semigrupo fortemente contínuo de operadores lineares. Definimos seu gerador infinitesimal como sendo o operador $A: D(A) \subset X \longrightarrow X$, dado por

$$
A x=\lim _{t \rightarrow 0^{+}} \frac{T(t) x-x}{t},
$$

onde $D(A)=\left\{x \in X: \exists \lim _{t \rightarrow 0^{+}} \frac{T(t) x-x}{t}\right\}$.

Surgem então duas perguntas naturais:

- dado um semigrupo $\{T(t): t \geq 0\}$, existe um único gerador infinitesimal?

- dado um gerador $A$ de um semigrupo $T(t)$, este semigrupo é único?

A resposta afirmativa à primeira pergunta é dada diretamente pela definição de operador infinitesimal. Já a, também afirmativa, resposta à segunda é dada pelo seguinte:

Teorema 6 Sejam $\{T(t): t \geq 0\}$ e $\{S(t): t \geq 0\}$ semigrupos fortementes contínuos gerados pelo operador $A: D(A) \subset X \longrightarrow X$, ou seja,

$$
\lim _{t \rightarrow 0^{+}} \frac{T(t) x-x}{t}=A x=\lim _{t \rightarrow 0^{+}} \frac{S(t) x-x}{t}, \forall x \in D(A) .
$$

Então, $T(t)=S(t)$ para todo $t \geq 0$.

Antes de demonstra-lo apresentaremos o seguinte teorema, que nos fornece uma importante e muito útil propriedade de semigrupos fortemente contínuos.

Teorema 7 Seja $\{T(t): t \geq 0\}$ um semigrupo fortemente continuo e A seu gerador infinitesimal. Então, para $x \in D(A)$, o operador

$$
t \mapsto T(t) x
$$

é continuamente diferenciável e

$$
\frac{d}{d t} T(t) x=A T(t) x=T(t) A x, \forall t \geq 0 .
$$

Demonstração. Seja $x \in D(A)$. Para provarmos que $T(t) x$ é continuamente diferenciável em $t$, é suficiente mostrar que a derivada a direita deste mesmo operador é contínua. Mostraremos inicialmente que $T(t) x \in D(A)$. Seja então $h>0$, segue que

$$
\frac{T(h)-I_{X}}{h} T(t) x=T(t)\left(\frac{T(h)-I_{X}}{h}\right) x \rightarrow T(t) A x, \text { se } h \rightarrow 0^{+} .
$$


Além de concluir que, de fato, $T(t) x \in D(A)$, obtemos que a derivada a direita de $T(t) x$ é contínua em $t$ e $A T(x)=T(t) A x$.

Demonstração do Teorema 6. Considere a aplicação $s \mapsto T(s) S(t-s)$, definida para $0 \leq s \leq t$. Se provarmos que a derivada desta aplicação é zero - e, portanto, ela é constante em $s$-, como ela vale $S(t)$ e $T(t)$ em $s=0$ e $s=t$, respectivamente, concluiremos que $S(t)=T(t), \forall t \geq 0$. Pelo teorema anterior, sabemos que esta aplicação é diferenciável e

$$
\frac{d}{d s}(T(s) S(t-s))=\frac{d T(s)}{d s} S(t-s)+T(s) \frac{d S(t-s)}{d s} .
$$

E então, ainda do teorema 7, obtemos

$$
\frac{d T(s)}{d s}=A T(s) e \frac{d S(t-s)}{d s}=-A S(t-s) .
$$

Portanto,

$$
\frac{d}{d s}(T(s) S(t-s)=A T(s) S(t-s)-T(s) A S(t-s)=A T(s) S(t-s)-A T(s) S(t-s)=0 .
$$

Usamos na última igualdade o fato de que $A$ comuta com $T(t)$.

Teorema 8 Sejam $T(t)$ um semigrupo fortemente contínuo de contrações e $A: D(A) \subset X \longrightarrow X$ seu gerador infinitesimal. Então são válidas as seguintes afirmações:

1. Para cada $x \in X, \lim _{h \rightarrow 0} \frac{1}{h} \int_{t}^{t+h} T(s) x d s=T(t) x$.

2. Para cada $x \in X, \int_{0}^{t} T(s) x d s \in D(A) e$

$$
A\left(\int_{0}^{t} T(s) x d s\right)=T(t) x-x
$$

3. Para cada $x \in D(A)$,

$$
T(t) x-T(s) x=\int_{s}^{t} T(\tau) A x d \tau=\int_{s}^{t} A T(\tau) x d \tau .
$$

\section{Demonstração.}

1. De acordo com o teorema 7, o operador $t \longmapsto T(t) x$ é contínuo, para cada $x \in X$. Então, $\lim _{h \rightarrow 0} \frac{1}{h} \int_{t}^{t+h} T(s) x d s=\lim _{h \rightarrow 0} \frac{1}{h}\left\{\int_{t}^{t+h} T(s) x d s-\int_{t}^{t} T(s) x d s\right\}=T(t) x$.

2. Seja $x \in X$, então

$$
\begin{gathered}
A\left(\int_{0}^{t} T(s) x d s\right)=\lim _{h \rightarrow 0} \frac{T(h)-I}{h}\left(\int_{0}^{t} T(s) x d s\right) \\
\quad=\lim _{h \rightarrow 0} \frac{1}{h} \int_{0}^{t}(T(s+h) x-T(s) x) d s
\end{gathered}
$$




$$
\begin{aligned}
& =\lim _{h \rightarrow 0} \frac{1}{h}\left\{\int_{t}^{t+h} T(s) x d s-\int_{0}^{h} T(s) x d s\right\} \\
& =T(t) x-x .
\end{aligned}
$$

Observe que na última igualdade aplicamos o item 1.

3. De acordo com o teorema 7 ,

$$
\frac{d}{d t} T(t) x=A T(t) x=T(t) A x, \forall t \geq 0
$$

Integrando de $s$ a $t$ obtemos o resultado.

Com o teorema a seguir obtemos outros importantes resultados acerca dos semigrupos fortemente contínuos e que nos serão úteis no decorrer deste capítulo. Em sua demonstração será utilizada a noção de $\sigma$-álgebra de Borel e mensurabilidade. Assim, primeiramente daremos as seguintes definições:

Definição 19 Dado um conjunto $X$, uma $\sigma$-álgebra sobre $X$ é uma coleção $\mathcal{X}$ de subconjuntos de $X$ tal que:

1. $X$ e $\emptyset$ estão contidos em $\mathcal{X}$.

2. Se uma subcoleção $\left\{X_{\alpha}, \alpha \in A\right\}$ está em $X$ então sua união também está em $X$.

3. Se $A \in \mathcal{X}$ então $A^{c} \in \mathcal{X}$.

A coleção

$$
\mathcal{R}=\{(a, b) \subset \mathbb{R} ; a<b\}
$$

é uma $\sigma$-álgebra sobre $\mathbb{R}$ e gera a chamada $\sigma$-álgebra de Borel.

Definição 20 Uma função $f: X \longrightarrow \mathbb{R}$ é dita $\mathcal{X}$-mensurável se, para todo $\alpha \in \mathbb{R}$ o conjunto

$$
\{x \in X ; f(x)>\alpha\}
$$

for um elemento da $\sigma$-álgebra $\mathcal{X}$.

Teorema 9 Seja $\{T(t): t \geq 0\}$ um semigrupo fortemente contínuo e A seu gerador infinitesimal. Então,

1. o operador $t \mapsto\|T(t)\|_{\mathcal{L}(X)}$ é semicontínuo inferiormente e, portanto, mensurável.

2. $\cap_{m \geq 1} D\left(A^{m}\right)$ é denso em $X$. 
3. se Re $\lambda>\beta$, onde $\beta$ é a constante dada pelo teorema 5, tem-se que $\lambda \in \rho(A)$ e

$$
(\lambda I-A)^{-1} x=\int_{0}^{\infty} e^{-\lambda t} T(t) x d t, \quad \forall x
$$

\section{Demonstração.}

1. Para garantirmos que $t \mapsto\|T(t)\|_{\mathcal{L}(X)}$, que denotaremos por $N$, é semicontínuo inferiormente, é suficiente mostrar que, para cada constante $r \geq 0$, o conjunto $A_{r}=\{t \geq 0:\|T(t) x\|>r\}$ é aberto em $[0, \infty)$. Seja então $t_{0} \in A_{r}$, ou seja, $\left\|T\left(t_{0}\right) x\right\|=\sup _{x \in B_{X}\left\|T\left(t_{0}\right) x\right\|}>r$. Sendo assim, existe pelo menos algum $x$ em $B_{X}$ tal que $\left\|T\left(t_{0}\right) x\right\|$. Segue, do teorema 7 , que existe uma vizinhança $V_{t_{0}}$ de $t_{0}$ tal que, para todo $t \in V_{t_{0}}$, tem-se $\left\|T\left(t_{0}\right) x\right\|>r$. Já que $r$ foi tomado arbitrariamente, provamos que $N$ de fato é semicontínuo inferiormente. Sabendo que $N$ é mensurável na $\sigma$-álgebra de Borel se, e somente se, para qualquer $\alpha \geq 0$, o conjunto $\{t \geq 0:\|T(t) x\|>\alpha\}$ for aberto em $[0, \infty)$, o resultado segue da semicontinuidade inferior de $N$.

2. Considere uma função $\phi$ de classe $\mathcal{C}^{\infty}(\mathbb{R})$ que anula um vizinhança da origem, i.e., tal que

$$
\phi(t)=0 \quad \forall t \in V_{0}
$$

e de suporte compacto. Fixamos um ponto $x \in X$ e definimos a aplicação

$$
f=\int_{0}^{\infty} \phi(t) T(t) x d t
$$

Então, a igualdade

$$
h^{-1}(T(h) f-f)=h^{-1} \int_{0}^{\infty}(\phi(t-h)-\phi(t)) T(t) x d t
$$

implica que $f \in D(A)$ e, mais ainda, que

$$
A f=-\int_{0}^{\infty} \phi^{\prime}(t) T(t) x d t .
$$

Assim, segue que para todo $m \geq 1$ e para toda $f \in \cap_{m \geq 1} D\left(A^{m}\right)$ obtemos que $A^{m}$ aplicado a $f$ relaciona-se com a $m$-ésima derivada de $\phi$ a menos de um sinal, i.e.,

$$
A^{m} f(x)=\int_{0}^{\infty} \phi^{(m)}(t) T(t) x d t
$$

Se tomarmos $\phi$ como sendo uma aproximação da unidade, ou seja,

$$
\int_{0}^{\infty} \phi(t) d t=1
$$


e definirmos nossa seqüência $f_{n}$ como

$$
f_{n}=\int_{0}^{\infty} n \phi(n t) T(t) x d t=\int_{0}^{\infty} \phi(s) T\left(\frac{s}{n}\right) x d s
$$

para todo $n$ natural, temos que cada $f_{n}$ é um ponto da interseção

$$
\cap_{m \geq 1} D\left(A^{m}\right)
$$

e claramente

$$
\left\|f_{n}-x\right\| \longrightarrow 0
$$

quando $n \longrightarrow \infty$. Da arbitrariedade de $x \in X$ segue a densidade.

3. Supondo que $\operatorname{Re}(\lambda)>\beta$ e que $\|T(t)\|<M e^{\beta t}$ e colocando

$$
R_{\lambda}(x)=\int_{0}^{\infty} e^{-\lambda t} T(t) x d t
$$

obtemos que

$$
\left\|R_{\lambda}\right\| \leq \frac{M}{\operatorname{Re}(\lambda)-\beta}
$$

Fixando $x \in X$ e $a \in \mathbb{R}^{+}$temos

$$
\begin{aligned}
a^{-1} & (T(A)-I) R_{\lambda}(x)=R_{\lambda} \frac{T(a) x-x}{a}= \\
& =a^{-1}\left[\int_{0}^{\infty} e^{-\lambda t+\lambda a} T(t) x d t-\int_{0}^{\infty} e^{-\lambda t} T(t) x d t\right]= \\
& =a^{-1}\left[-\int_{0}^{\infty} e^{\lambda(a-t)} T(t) x d t+\int_{0}^{\infty}\left(e^{\lambda a}-1\right) e^{-\lambda t} T(t) x\right]= \\
& \longrightarrow-x+\lambda R_{\lambda}(x),
\end{aligned}
$$

quando $a \longrightarrow 0^{+}$. Assim, concluímos que $R_{\lambda}(x)$ pertence ao domínio de $A, D(A)$. Mais ainda, $(\lambda I-A) R_{\lambda}(x)=x$ e $\lambda I-A$ é sobrejetor. Por outro lado, tomando $x \in D(A)$, vemos que $A R_{\lambda}(x)=R_{\lambda}(A x)$ implica a injetividade de $(\lambda I-A)$, uma vez que

$$
\left.(\lambda I-A) R_{\lambda}(x)\right)=x=R_{\lambda}(\lambda I-A) x .
$$

Com isto, estabelece-se que $(\lambda I-A)$ é uma bijeção do domínio de $A$ sobre $X$ cuja inversa é limitada, o que implica no resultado desejado.

Definição 21 Seja $\{T(t): t \geq 0\}$ um semigrupo fortemente contínuo. Se existir uma constante $M$ tal que $\|T(t)\| \leq M, \forall t \geq 0$, dizemos que $T(t)$ é um semigrupo uniformemente limitado. Em 
particular, se $M=1$, dizemos que $T(t)$ é um semigrupo de contrações.

\subsubsection{Os teoremas de Hille-Yosida e Lumer-Phillips}

Como foi discutido nas subseções anteriores, todo semigrupo fortemente contínuo possui um gerador infinitesimal. Outra questão também importante é reconhecer tais geradores. O objetivo desta subseção é apresentar dois resultados cruciais acerca desta caracterização. Os Teoremas de Hille-Yosida e de Lumer-Phillips nos fornecem condições necessárias e suficientes para que um operador gere ${ }^{10}$ um semigrupo fortemente contínuo, no caso do segundo, um semigrupo fortemente contínuo de contrações. Tal caracterização acerca dos geradores nos será bastante útil no estudo de soluções do problema de Cauchy abstrato. Antes de apresentarmos estes resultados, discutiremos alguns conceitos e dois lemas que nos serão úteis, principalmente na demonstração do Teorema de Hille-Yosida.

No caso geral o operador linear $A: D(A) \subset X \longrightarrow X$ não é contínuo, mas se ele satisfizer algumas hipóteses - que serão apresentadas no próximo teorema - é possível encontrar uma seqüência de operadores em $\mathcal{L}(X)$ que converge pontualmente para $A$. Esta é uma propriedade bastante útil no sentido em que ela contorna diversos problemas acerca de $A$, gerados pelo fato de este não ser um operador limitado.

Definição 22 Sejam $X$ um espaço de Banach e $A: D(A) \subset X \longrightarrow X$ um operador linear fechado. A aproximação de Yosida de A é dada por

$$
A_{\lambda} \doteq \lambda A(\lambda I-A)^{-1}, \text { para } \lambda \in \rho(A)
$$

Vale ressaltar que $A_{\lambda}$ é um operador linear e limitado em $X$. De fato, sendo $(\lambda I-A)(\lambda I-A)^{-1}=$ $I$, obtemos

$$
A_{\lambda}=\lambda^{2}(\lambda I-A)^{-1}-\lambda I
$$

que é a soma de dois operadores em $\mathcal{L}(X)$.

Lema 8 Sejam $X$ um espaço de Banach e $A: D(A) \subset X \longrightarrow X$ um operador linear fechado $e$ densamente definido. Assuma que $\lambda \in \rho(A)$, para todo $\lambda>0$ e que

$$
\left\|(\lambda I-A)^{-1}\right\| \leq \frac{1}{\lambda}, \forall \lambda>0 .
$$

Então são válidas as seguintes afirmações:

1. $\lim _{\lambda \rightarrow \infty} \lambda(\lambda I-A)^{-1} x=x, \forall x \in X$

2. $\lim _{\lambda \rightarrow \infty} A_{\lambda} x=A x, \forall x \in X$;

3. para cada $\lambda>0$, a aproximação de Yosida $A_{\lambda}$ gera o semigrupo fortemente contínuo $e^{A_{\lambda} t}$.

\footnotetext{
${ }^{10}$ Quando dizemos que $A$ gera um semigrupo qualquer $T(t)$, queremos dizer que $A$ é o gerador infinitesimal de $T(t)$.
} 
Lema 9 Sejam $X$ um espaço de Banach e $A: D(A) \subset X \longrightarrow X$ o gerador do semigrupo fortemente contínuo $T(t)$ tal que

$$
\|T(t)\| \leq M e^{\beta t}, \quad \forall t \geq 0 .
$$

Seja ainda $\alpha \in \mathbb{R}$. Então se $\tilde{T}(t)=T(t) e^{-\alpha t}$, são válidas as seguintes afirmações:

1. $\tilde{T}(t)$ é também um semigrupo fortemente contínuo e ainda satisfaz

$$
\|\tilde{T}(t)\| \leq M e^{(\beta-\alpha) t}, \quad \forall t \geq 0
$$

2. $\tilde{A}=A-\alpha I$ é o gerador infinitesimal de $\tilde{T}$ e $D(A)=D(\tilde{A})$;

3. $\rho(\tilde{A})=\rho(A)-\alpha e(\lambda-\tilde{A})=(\lambda+\alpha-A)$.

Teorema 10 [Hille-Yosida] Sejam $X$ um espaço de Banach e $A: D(A) \subset X \longrightarrow X$ um operador linear. Então, as seguintes afirmações são equivalentes:

(i) A é o operador infinitesimal de um semigrupo fortemente contínuo $T(t)$ em $\mathcal{L}(X)$ tal que

$$
\|T(t)\| \leq e^{\beta t}, \quad \forall t \geq 0
$$

(ii) A é fechado, densamente definido, o intervalo $(\beta, \infty)$ está contido no resolvente de $A$ e

$$
\left\|(\lambda-A)^{-1}\right\| \leq \frac{1}{\lambda-\beta}, \forall \lambda>\beta
$$

onde $\beta$ é a constante dada pelo teorema 5.

Demonstração. Para verificarmos que $(i) \Rightarrow(i i)$, tomemos $x \in X$ e mostremos que existe uma seqüência em $D(A)$ que converge para $x$. Considere, para cada $t>0, x_{t}=\frac{1}{t} \int_{0}^{t} T(s) d s$. Os ítens 2 e 1 do teorema 8 nos garantem, respectivamente, que $x_{t} \in D(A)$, para cada $t>0$, e que $x_{t} \rightarrow x$ quando $t \rightarrow 0$. De onde segue que $\overline{D(A)}=X$. Considere agora uma seqüência $x_{n}$ em $D(A)$ tal que $x_{n}$ e $A x_{n} \rightarrow y$. Obtemos do item 3 do mesmo teorema 8 que,

$$
T(t) x_{n}-x_{n}=\int_{0}^{t} T(s) A x_{n} d s
$$

E, portanto, quando $n \rightarrow \infty$, temos

$$
T(t) x-x=\int_{0}^{t} T(s) y d s
$$

pois $T(s) A x_{n}$ converge uniformemente para $T(s) y$ em cada intervalo limitado. Consequentemente,

$$
\lim _{t \rightarrow 0} \frac{T(t) x-x}{t}=\lim _{t \rightarrow 0} \frac{1}{t} \int_{0}^{t} T(s) y d s=T(0) y=y
$$


novamente pelo item 1 do teorema 8. E então, $x \in D(A)$ e $A x=y$. Finalmente, pelo item 3 do teorema 9, obtemos

$$
\lambda \in \rho(A), \forall \lambda \in(\beta, \infty)
$$

$\mathrm{e}$

$$
\left\|(\lambda I-A)^{-1} x\right\| \leq \int_{0}^{\infty}\left\|e^{\lambda t} e^{\beta t} x\right\| d t \leq \frac{1}{\lambda-\beta}, \forall \lambda>\beta .
$$

Reciprocamente, suponha que a afirmação (ii) seja válida. Tomando $\alpha=\beta$ no lema 9 , concluímos que é suficiente que seja provado o resultado para o caso em que $\beta=0$. Queremos então encontrar um semigrupo fortemente contínuo $T(t)$ em $\mathcal{L}(X)$ tal que $\|T(t)\| \leq 1$, para todo $t>0$, e $A$ seja seu gerador infinitesimal.

Considere, para cada $\lambda>0$, a aproximação de Yosida $A_{\lambda}$ de $A$. Como vimos no item 3 do lema $8, A_{\lambda}$ gera o semigrupo fortemente contínuo $e^{A_{\lambda} t}$ e vale ainda que

$$
\left\|e^{A_{\lambda t}}\right\| e\left\|e^{A_{\lambda} t} x-e^{A_{\mu} t}\right\| \leq t\left\|A_{\lambda} x-A_{\mu} x\right\|
$$

para todo $\lambda>0$ e $\mu>0$. Definiremos então, para cada $x \in D(A), T(t) \doteq \lim _{\lambda \rightarrow \infty} e^{A_{\lambda t}}$. Verifiquemos que $T(t)$ está bem definido e que, de fato, corresponde a um semigrupo fortemente contínuo de contrações. Seja $x \in X$, então, para $\lambda>0$ e $\mu>0$, vale que

$$
\left\|e^{A_{\lambda} t} x-e^{A_{\mu} t}\right\| \leq t\left\|A_{\lambda} x-A x\right\|+t\left\|A_{\mu} x-A x\right\| .
$$

Além disso, $\lim _{A_{\lambda}} x=A x$, para cada $x \in X$. Concluímos assim que $e^{A_{\lambda t}} x$ converge uniformemente em qualquer intervalo limitado contido no semi-eixo positivo de $\mathbb{R o}$ limite existe e, portanto, $T(t)$ está bem definido. Sendo $\left\|e^{A_{\lambda} t}\right\| \leq 1$, pode-se obter que $T(t) \in \mathcal{L}(X)$, pois $\|T(t) x\| \leq\|x\|$, para $x \in X$. Como $A$ é um operador densamente definido, existe uma única extensão $T(t)$ para $X$ com $\|T(t)\| \leq 1$. As propriedades 1 e 2 da definição de semigrupos são diretamente verificadas. Veremos agora que o item 4 também é satisfeito. Sejam então $x \in X$ e $\epsilon>0$. Existem assim $\bar{x} \in D(A)$ e $\bar{t}$ tais que, $\|\bar{x}-x\|<\frac{\epsilon}{3}$ e $\|T(t) \bar{x}-\|<\frac{\epsilon}{3}$, se $0 \leq t \leq \bar{t}$. De onde segue que

$$
\|T(t) x-x\| \leq\|T(t) x-T(t) \bar{x}\|+\|T(t) \bar{x}-\bar{x}\|+\|\bar{x}-x\|<\epsilon .
$$

O próximo e último passo é provar que $A$ é o gerador de $T(t)$. Suponha que o operador $B: D(B) \subset X \longrightarrow X$ seja o gerador infinitesimal de $T(t)$. De acordo com o item 3 do teorema 8 e com o item 2 do lema 8 , se $x \in D(A)$, então

$$
T(t) x-x=T(t) x-T(0) x=\lim _{\lambda \rightarrow \infty} \int_{0}^{t} e^{A_{\lambda} s} A_{\lambda} x d s=\int_{0}^{t} T(s) A x d s .
$$

Segue então, da definição de gerador infinitesimal e do item 3 do teorema 8 que, se $x \in D(A)$, temos que $x \in D(B)$ e ainda que $B x=A x$. Ou seja, o gerador $B$ é uma extensão de $A$. Nos resta provar então que $D(A) \subset D(B)$. Por hipótese, $1 \in \rho(A)$. Sendo $B$ o gerador infinitesimal de um 
semigrupo $T(t)$ tal que $\|T(t)\| \leq 1$, vale ainda que $1 \in \rho(B)$. Pela definição do conjunto resolvente, obtemos

$$
X=(I-A) D(A)=(I-B) D(B),
$$

e, portanto,

$$
(I-B) D(A)=X=(I-B) D(B) .
$$

De onde segue que, $D(B)=(I-B)^{-1} X=(I-B)^{-1}(I-B) D(A)=D(A)$ e concluímos o resultado.

Observe que, se o semigrupo do teorema anterior for um semigrupo de contrações, então $\beta=1$, pois $\|T(t)\| \leq 1=M e^{0 t}, \forall t \geq 0$. Nestas condições, o item (ii) pode ser reformulado da seguinte forma:

$\left(i i^{\prime}\right) \rho(A)$ contém o semi-eixo positivo e, para todo $\lambda>0$, tem-se

$$
\left\|(\lambda-A)^{-1}\right\| \leq \frac{1}{\lambda}
$$

O Teorema de Hille-Yosida, na forma como foi enunciado acima, depende da norma em $X$. Mas vamos verificar, no próximo lema, que sempre existe uma norma em $X$, de modo que as condições (i) e (ii) sejam verificadas. O que existe na verdade é uma forma mais geral deste mesmo teorema, como veremos, que independe da norma.

Lema 10 Sejam $X$ um espaço de Banach e $A: D(A) \subset X \longrightarrow X$ um operador linear tal que $(0, \infty) \subset \rho(A) e$

$$
\left\|(\lambda I-A)^{-n}\right\| \leq M \lambda^{-n}, \text { para } n=1,2,3, \ldots \text { e } \forall \lambda>0,
$$

onde $M \geq 1$ é constante. Então existe uma norma $|$.$| em X$ tal que

$$
\|x\| \leq|x| \leq M\|x\|, \quad \forall x \in X
$$

e ainda que

$$
\left|(\lambda I-A)^{-1} x\right| \leq \frac{|x|}{\lambda}, \quad \forall x \in X, \quad \forall \lambda>0 .
$$

Demonstração. Se $\mu>0$ e $|\mu-\lambda|<\mu$ então

$$
(\lambda I-A)^{-1}=(\lambda I-\mu I+(\mu I-A))^{-1}=\sum_{k=0}^{\infty}(\mu I-\lambda)^{k}(\mu I-A)^{-k-1} .
$$

Afirmamos que a série acima converge, uma vez que

$$
\left\|(\mu-\lambda)^{k}(\mu I-A)^{-k-1}\right\| \leq M \frac{|\mu-\lambda|^{k}}{\mu^{k+1}} \longrightarrow 0
$$


se $k \rightarrow \infty$. O mesmo vale, em particular, para $0<\lambda<\mu$ e, como

$$
\begin{gathered}
\frac{1}{p !}\left(\frac{d}{d \lambda}\right)^{p}(\lambda I-A)^{-1}=(-1)^{p}(\lambda I-A)^{-p-1} \\
\quad=\sum_{k=p}^{\infty}(-1)^{k} \frac{k !(\mu-\lambda)^{k-p}}{p !(k-p) !}(\lambda I-A)^{-k-1}
\end{gathered}
$$

então $(\lambda I-A)^{-p-1}=\sum_{k=p}^{\infty} \frac{k !(\lambda-\mu)^{k-p}}{p !(k-p) !}(\lambda I-A)^{-k-1}$ e, para $0<\lambda<\mu$, vale que

$$
\left\|\lambda^{p+1}(\lambda I-A)^{-p-1} x\right\| \leq \frac{k !}{p !(k-p) !}\left(\frac{\mu-\lambda}{\mu}\right)^{k-p}\left(\frac{\lambda}{\mu}\right)^{p+1}\left\|\mu^{k+1}(\mu I-A)^{-k-1} x\right\| .
$$

Definindo $\|x\|_{\mu}=\sup _{n \geq 0}\left\|\mu^{n}(\lambda I-A)^{-n} x\right\|$ para $\mu>0$, obtemos $\|x\| \leq\|x\|_{\mu} \leq M\|x\|$. Além disso, para $0<\lambda<\mu,\|x\|_{\lambda} \leq\|x\|_{\mu}$, já que

$$
\left\|\lambda^{p+1}(\lambda I-A)^{p-1} x\right\| x \leq \sum_{k=p}^{\infty}\left(\begin{array}{l}
k \\
p
\end{array}\right)\left(\frac{\mu-\lambda}{\mu}\right)^{k-p}\left(\frac{\lambda}{\mu}\right)^{p+1}\|x\|_{\mu}=\|x\|_{\mu} .
$$

Uma vez que a aplicação que leva $\lambda$ a $\|x\|_{\lambda}$ é crescente, e limitada superiormente, e, mais ainda, toma valores no corpo escalar completo, existe $\sup _{\lambda>0}\|x\|_{\lambda}$. Seja

$$
|x|_{X}=\sup _{\lambda>0}\|x\|_{\lambda}
$$

Para verificarmos que $|x|_{X}$ é uma norma em $X$ basta notarmos que $\|x\|_{\lambda}$ é uma norma e, consequentemente, verificam-se as propriedades desejadas para $|x|_{X}$.

Segue que

$$
\|x\|_{X} \leq|x|_{X} \leq\|x\|_{X}
$$

o que implica na equivalência destas normas; mais ainda, se $\lambda \in(0, \mu)$ temos que

$$
\begin{array}{r}
\left\|\mu^{p}(\mu I-A)^{-p} \lambda(\lambda I-A)^{-1} x\right\|_{X}=\left\|\lambda(\lambda I-A)^{-1} \mu^{p}(\mu I-A)^{-p} x\right\|_{X} \leq \\
\leq\left\|\mu^{p}(\mu I-A)^{-p} x\right\|_{\lambda} \leq\left\|\mu^{p}(\mu I-A)^{-p} x\right\|_{\mu} \leq\|X\|_{\mu} \leq|x|_{X} .
\end{array}
$$

Segue imediatamente que

$$
\left\|\lambda(\lambda I-A)^{-1} x\right\|_{\mu} \leq|x|_{X}
$$

e

$$
\left|\lambda(\lambda I-A)^{-1} x\right|_{X} \leq|x|_{X}
$$

Teorema 11 (Forma Geral do Teorema de Hille-Yosida) Sejam X um espaço de Banach e 
$A: D(A) \subset X \longrightarrow X$ um operador linear. Então, as seguintes afirmações são equivalentes:

(i) A é o operador infinitesimal de um semigrupo fortemente contínuo $T(t)$ em $\mathcal{L}(X)$ tal que

$$
\|T(t)\| \leq M e^{\beta t}, \quad \forall t \geq 0 .
$$

(ii) A é fechado, densamente definido, o intervalo $(\beta, \infty)$ está contido no resolvente de $A$ e

$$
\left\|(\lambda-A)^{-n}\right\| \leq \frac{M}{(\lambda-\beta)^{n}}, \quad \forall \lambda>\beta, n=1,2,3, \ldots
$$

onde $\beta$ é a constante dada pelo teorema 5.

Demonstração. Da mesma forma como foi feita na demonstração do teorema 10, podemos supor, sem perda de generalidade, que $\beta=0$. Para verificarmos que $(i) \Rightarrow(i i)$, observamos que, de acordo com o item 3 do teorema 9 , vale que $(0, \infty) \subset \rho(A)$ e

$$
(\lambda I-A)^{-1} x=\int_{0}^{\infty} e^{-\lambda t} T(t) x d t, \quad \forall \lambda>0 .
$$

Derivando $3.10 p+1$ vezes, em relação a $\beta$, obtemos

$$
(-1)(-2) \ldots(-p)(\lambda I-A)^{-p-1} x=\int_{0}^{\infty} e^{-\lambda t}(-t)^{p} T(t) x d t
$$

Portanto,

$$
\left\|(\lambda I-A)^{-p-1} x\right\| \leq \frac{M}{p !} \int_{0}^{\infty} e^{-\lambda t} t^{p} d t\|x\|=\lambda^{-p-1} M\|x\|,
$$

para $\mathrm{p}=0,1,2, \cdots$. Suponha agora que a afirmação $(i i)$ seja válida. Como vimos, é suficiente supor para $\beta=0$. Sabemos que existe uma norma $|$.$| em X$, que é equivalente a $\|\cdot\|$, tal que

$$
\|x\| \leq|x| \leq M\|x\|, \quad \forall x \in X
$$

e ainda que

$$
\left|(\lambda I-A)^{-1}\right| \leq \frac{|x|}{\lambda}, \forall x \in X, \forall \lambda>0 .
$$

Concluímos que $A$ gera um semigrupo fortemente contínuo $T(t)$ tal que $|T(t) x| \leq|x|$. De onde segue que

$$
\|T(t) x\| \leq|T(t) x| \leq|x| \leq M\|x\|
$$

Uma formulação alternativa ao Teorema de Hille-Yosida, conhecida como Teorema de LumerPhillips, nos fornece uma caracterização distinta de geradores de semigrupos fortemente contínuos, desta vez de contrações, e em termos de operadores dissipativos.

Teorema 12 (Lumer-Phillips) Seja $A: D(A) \subset X \longrightarrow X$ um operador linear tal que $\overline{D(A)}=$ 
X. São válidas as seguintes afirmações:

1. Se $A$ é dissipativo e existe algum $\lambda_{0}>0$ tal que $R\left(\lambda_{0}-A\right)=X$, então $A$ é o gerador de um semigrupo fortemente contínuo de contrações em $X$.

2. Se A é gerador de um semigrupo fortemente contínuo de contrações em $X$, então $A$ é dissipativo e $R(\lambda-A)=X$, para todo $\lambda>0$. Além disso, para todo $x \in D(A), \operatorname{Re}\left\langle x^{*}, A x\right\rangle \leq 0$, para todo $x^{*} \in J(x)$.

\section{Demonstração.}

1. Uma vez que $A$ é dissipativo, é possível obter do lema 6 que, para todo $\lambda>0$ e todo $x \in D(A)$,

$$
\|(\lambda I-A) x\| \geq \lambda\|x\|
$$

Em particular 3.11 é válido para $\lambda=\lambda_{0}$. De onde segue que

$$
\left\|\left(\lambda_{0} I-A\right)^{-1}\right\| \leq \lambda_{0}
$$

já que, por hipótese, $\lambda_{0} I-A$ é inversível em $X$. Logo o operador linear $\left(\lambda_{0} I-A\right)^{-1}$ é limitado e, portanto, fechado. De onde também obtemos que $A$ é fechado. Podemos então aplicar o teorema 4 e concluir que $(0, \infty) \subset \rho(A)$ e $\left\|(\lambda I-A)^{-1}\right\| \leq \frac{1}{\lambda}$. Ficam assim verificadas todas as hipóteses do Teorema de Hille-Yosida para o operador $A$. Portanto, $A$ é gerador infinitesimal de um semigrupo fortemente contínuo de contrações.

2. Sendo $A$ um gerador infinitesimal de um semigrupo fortemente contínuo de contrações, obtemos, pelo Teorema de Hille-Yosida, que $(0, \infty) \subset \rho(A)$ e que $\left\|(\lambda I-A)^{-1}\right\| \leq \frac{1}{\lambda}$. Tais afirmações implicam, respectivamente, que $R(\lambda I-A)=X$, para todo $\lambda>0$, e que $A$ é dissipativo. Tomemos agora um elemento qualquer $x$ no domínio de $A$. Segue-se, pela definição de operador dissipativo e pela desigualdade de Cauchy-Schwartz, que

$$
R e\left\langle x^{*}, T(t) x\right\rangle<\left|\left\langle x^{*}, T(t) x\right\rangle\right| \leq\left\|x^{*}\right\|\|T(t) x\| \leq\|x\|^{2}
$$

para todo $x^{*} \in J(x)$. De onde obtemos,

$$
R e\left\langle x^{*}, T(t) x-x\right\rangle=R e\left\langle x^{*}, T(t) x\right\rangle-\|x\|^{2} \leq 0 .
$$

Dividindo por $t$ e tomando o limite quando $t$ tende para 0 , concluímos que $\operatorname{Re}\left\langle x^{*}, A x\right\rangle \leq 0$.

A hipótese de $A$ ser densamente definido é importante quando queremos usar alguns resultados como o de Lumer-Phillips. O próximo teorema nos fornece condições acerca de um operador dissipativo que garantem que ele esteja densamente definido.

Teorema 13 Se $A$ é dissipativo em $X, R\left(I_{X}-A\right)=X$ e $X$ for reflexivo então $\overline{D(A)}=X$. 
Demonstração. Fixemos um funcional linear definido sobre $X$, ou seja, um ponto $x^{*} \in X^{*}$ tal que $\operatorname{ker}\left(x^{*}\right) \supset D(A)$. Vamos mostrar que $\operatorname{ker}\left(x^{*}\right)=X$.

Uma vez que $R\left(I_{X}-A\right)=X$ é suficiente mostrarmos que $\left\langle x^{*}, x-A X\right\rangle=0$ para todo $x \in D(A)$. Como $\left\langle x^{*}, x\right\rangle=0$ para todo $x \in D(A)$, e da linearidade de $x^{*}$, segue que $\left\langle x^{*}, x-A x\right\rangle=\left\langle-x^{*}, A x\right\rangle$. Fixemos $x$ em $D(A)$. Pelo teorema anterior, existe um $x_{n}$ tal que

$$
x=x_{n}-\left(\frac{1}{n}\right) A x_{n} .
$$

Como o ponto $A x_{n}=n\left(x_{n}-x\right) \in D(A)$, temos que $x_{n} \in D\left(A^{2}\right)$ e $A x=A x_{n}-\left(\frac{1}{n}\right) A^{2} x_{n}$ ou $\left(I_{X}-\left(\frac{1}{n}\right) A\right) A x_{n}=A x$.

A partir do lema 6 obtemos que

$$
\left\|A x_{n}\right\|_{X} \leq\|A x\|_{X}
$$

Assim,

$$
\left\|x_{n}-x\right\|_{X} \leq\left(\frac{1}{n}\right)\left\|A x_{n}\right\|_{X} \leq\left(\frac{1}{n}\right)\|A x\|_{X}
$$

$\mathrm{e}$

$$
x_{n} \longrightarrow x,
$$

quando $n \longrightarrow \infty$.

Da reflexividade de $X$, se necessário passando a uma subseqüência, $A x_{n}$ converge fracamente para, digamos, $f$. Por ser $A$ fechado, segue que $A x=f$. Assim,

$$
\left\langle x^{*}, A x_{n}\right\rangle=n\left\langle x^{*}, x_{n}-x\right\rangle=0
$$

a partir da continuidade e da linearidade de $x^{*}$. Pela mesma razão, fazendo $n \longrightarrow \infty$ temos que

$$
\left\langle x^{*}, A x\right\rangle=0 .
$$

Assim, vemos que $x^{*} \equiv 0$ em $X$ e $\overline{D(A)}=X$.

Se retirarmos, do teorema acima, a hipótese de $X$ ser reflexivo, então $A$ pode não ser densamente definido. Para um exemplo que ilustrar este fato, remetemos o leitor à página 29 de [23].

\subsubsection{Operadores setoriais e semigrupos analíticos}

Semigrupos analíticos constituem uma importante classe dos semigrupos fortemente contínuos, principalmente no que diz respeito a dinâmica de sistemas de dimensão infinita. Tal classe de semigrupos é fundamental, como veremos na última seção deste capítulo e ainda no próximo, para o estudo de sistemas parabólicos não-lineares.

Como a definição de semigrupo analítico envolve o conceito de função analítica, apresentamos a seguinte 
Definição 23 Sejam $X$ um espaço de Banach, $\Omega$ um subconjunto aberto de $\mathbb{C}$ e $g: \Omega \subset \mathbb{C} \longrightarrow X$. Dizemos que g é uma função analítica se existir, para todo $z \in \Omega$, o limite

$$
\lim _{h \rightarrow 0} \frac{g(z+h)-g(z)}{h},
$$

onde $h \rightarrow 0$ denota o limite, em $\mathbb{C}$, quando $h$ tende a origem, ou seja, pode ser interpretado como $|h| \rightarrow 0$.

O termo analítico vem do fato de que é possível considerar semigrupos cujo domínio do parâmetro $t$ pode ser estendido ao plano complexo. Para que ainda assim suas propriedades sejam preservadas, é suficiente que o domínio no qual o parâmetro complexo será definido seja um semigrupo aditivo, ou seja, um conjunto com a uma relação binária (adição) que satisfaz a propriedade associativa.

Não é necessário, portanto, que o semigrupo seja estendido a todo o plano complexo. Como veremos na definição, é suficiente estendê-lo a um setor deste mesmo plano. Apresentaremos então, antes de definirmos semigrupos analíticos, algumas notações e uma propriedade importante de alguns setores em $\mathbb{C}$.

Dados $\theta, \sigma \in(0, \pi)$, considere os seguintes setores:

- $\Delta_{\theta} \doteq\{z \in \mathbb{C}:|\arg z|<\theta, z \neq 0\}$

- $\Delta_{\theta}(a) \doteq a+\Delta_{\theta}=\{z \in \mathbb{C}:|\arg (z-a)|<\theta, z \neq a\}$

- $\Sigma_{\sigma} \doteq\{z \in \mathbb{C}:|\arg z|>\sigma, z \neq 0\}$

- $\Sigma_{\sigma}(a) \doteq a+\Sigma_{\sigma}=\{z \in \mathbb{C}:|\arg (z-a)|>\sigma, z \neq a\}$

No caso $\theta+\sigma=\pi$, vale ainda a relação $\Delta_{\theta}(-a)=-\Sigma_{\sigma}(a)$ já que

$$
-\Sigma_{\sigma}(a)=-a-\Sigma_{\sigma}=-a+\{-z \in \mathbb{C}:|\arg z|>\sigma, z \neq 0\}=-a+\Delta_{\theta}=\Delta_{\theta}(-a) .
$$

Observamos que acima foi usado o fato de que $-\Sigma_{\sigma}$ é a reflexão, em relação à origem, do setor $\Sigma_{\sigma}$. Segue abaixo um esboço de alguns deles.

Definição 24 Dizemos que um semigrupo fortemente contínuo $\{T(t): t \geq 0\} \subset \mathcal{L}(X)$, onde $X$ é um espaço de Banach, é um semigrupo analítico se o domínio do parâmetro $t$ puder ser estendido a um setor da forma $\Delta_{\theta} \cup 0$, para algum $\theta \in\left(0, \frac{\pi}{2}\right)$ e, além disso, forem satisfeitas as seguintes afirmações

1. a extensão de $T(t)$ é ainda um semigrupo fortemente contínuo

2. a aplicação $R: \Delta_{\theta} \longrightarrow X$ dada por $R(t)=T(t) x$ é analítica em $\Delta_{\theta}$, para cada $x \in X$.

É possível ainda caracterizar os geradores infinitesimais de semigrupos analíticos. Estes são conhecidos na literatura como operadores setoriais e a definição é dada a seguir. 


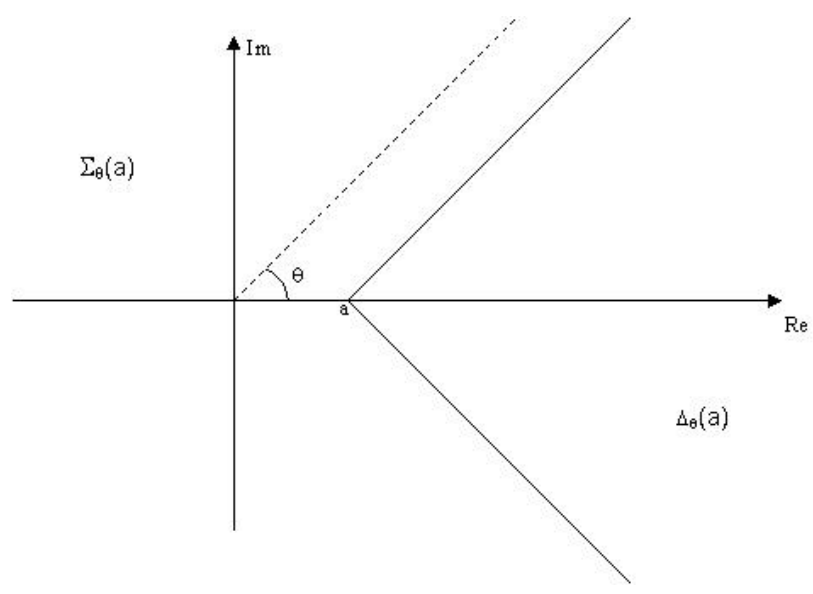

Figura 3.2: Setores $\Sigma_{\theta}(a)$ e $\Delta_{\theta}(a)$.

Definição 25 Sejam $X$ um espaço de Banach sobre um corpo $\mathbb{K}$ e $A: D(A) \subset X \longrightarrow X$ um operador linear fechado e densamente definido. Dizemos que A é um operador setorial se existem constantes $\sigma \in\left(0, \frac{\pi}{2}\right), M \geq 1$ e $a \in \mathbb{R}$ tais que o setor $\Sigma_{\sigma}(a)$ está contido no resolvente de $A$ e, além disso, a desigualdade

$$
\left\|(\lambda I-A)^{-1}\right\| \leq \frac{M}{|\lambda-a|}
$$

é satisfeita, para todo $\lambda \in \Sigma_{\sigma}(a)$.

Teorema 14 Sejam $X$ Banach e $A: D(A) \subset X \longrightarrow X$ um operador linear e densamente definido. Então $A_{\omega}=A+\omega I$ é um operador setorial, para todo $\omega \in \mathbb{R}$, se, e somente se, existem constantes $M \geq 1, a, b$ tais que $\rho\left(A_{b}\right)$ contém o conjunto $\{\lambda \in \mathbb{C}: \operatorname{Re} \lambda<a\}$ e $\|\lambda(\lambda I-(A+b I))\| \leq M$ para todo $\lambda \in \mathbb{C}$ com $\operatorname{Re} \lambda<a$.

Provaremos a seguir dois resultados que nos fornecem uma grande variedade de exemplos de operadores setoriais. O primeiro diz que todo operador linear e limitado em um espaço de Banach é setorial. Considere, por exemplo, o espaço de Banach $l_{2}=\left\{\left(x_{n}\right)_{n}\right.$ em $\left.\mathbb{C}: \sum_{i=1}^{\infty}\left|x_{i}\right|^{2}<\infty\right\}$, com a norma $\left\|\left(x_{n}\right)_{n}\right\|_{2}=\left(\sum_{i=1}^{\infty}\left|x_{i}\right|^{2}\right)^{\frac{1}{2}}$ e o operador $S_{r}: l_{2} \longrightarrow l_{2}{ }^{11}$, conhecido como operador deslocamento a direita, dado por

$$
S_{r}\left(x_{1}, x_{2}, \cdots, x_{n}, \cdots\right)=\left(0, x_{1}, x_{2}, \cdots, x_{n}, \cdots\right)
$$

para qualquer seqüência $\left(x_{1}, x_{2}, \cdots, x_{n}, \cdots\right) \in l_{2}$. $S_{r}$ é um operador setorial uma vez que ele é linear $\mathrm{e}$

$\left\|S_{r}\left(x_{1}, x_{2}, \cdots, x_{n}, \cdots\right)\right\|_{2}=\left\|\left(0, x_{1}, x_{2}, \cdots, x_{n}, \cdots\right)\right\|_{2}=\left\|\left(x_{1}, x_{2}, \cdots, x_{n}, \cdots\right)\right\|_{2}, \quad \forall\left(x_{1}, x_{2}, \cdots, x_{n}, \cdots\right) \in l_{2}$

\footnotetext{
${ }^{11}$ Utilizamos aqui a notação $S_{r}$ para o operador de deslocamento à direita em consoância com a notação presente na literatura em inglês, na qual o mesmo operador é chamado Right Shift operator.
} 
Teorema 15 Se $X$ é um espaço de Banach e $A: X \longrightarrow X$ é um operador linear e limitado, então A é setorial.

Demonstração. Sendo $A$ um operador linear e limitado, obtemos, pelo teorema 2, que

$$
\{\lambda \in \mathbb{C}:|\lambda|>\|A\|\} \subset \rho(A)
$$

e

$$
(\lambda I-A)^{-1}=\sum_{n=0}^{\infty} \frac{A^{n}}{\lambda^{n+1}},
$$

para cada $\lambda \in \mathbb{C}$ com $|\lambda|>\|A\|$. Em particular, $\{\lambda \in \mathbb{C}: \operatorname{Re} \lambda<-2\|A\|\} \subset \rho(A)$. Uma vez que $-|\lambda| \leq \operatorname{Re} \lambda$ para todo $\lambda \in \mathbb{C}$, é possível concluir que para $\lambda \in \mathbb{C}$ com $\operatorname{Re} \lambda<-2\|A\|$ vale que $-|\lambda|<-2\|A\|$, de onde obtemos $\frac{\|A\|}{|\lambda|}<\frac{1}{2}$. Assim sendo,

$$
\|\lambda(\lambda I-A)\| \leq \sum_{n=0}^{\infty}\left(\frac{\|A\|}{|\lambda|}\right)^{n} \leq \sum_{n=0}^{\infty}\left(\frac{1}{2}\right)^{n}=2 .
$$

Aplicando o teorema 14, concluímos que $A$ é um operador setorial em $X$.

Segue um resultado que diz que operadores definidos em espaços de Hilbert que são autoadjuntos e limitados inferiormente também são exemplos de operadores setoriais.

Teorema 16 Sejam $H$ um espaço de Hilbert, com o produto interno $\langle\cdot, \cdot\rangle$ e a norma $\|\cdot\|$, e o operador auto-adjunto $A: D(A) \subset H \longrightarrow H$. Se A for limitado inferiormente, ou seja, existe uma constante $m$ tal que

$$
\langle A x, x\rangle \geq m\|x\|^{2}, \quad \forall x \in D(A),
$$

então A é um operador setorial e, além disso, Re $\lambda \geq m$ para todo $\lambda \in \sigma(A)$.

Demonstração. De acordo com a proposição $3, A$ é um operador fechado, uma vez que ele é autoadjunto. Além disso, o teorema 3 nos garante $\sigma(A) \subset[m, \infty)$ e, em particular, $\Sigma_{\frac{\pi}{4}}(m) \subset \rho(A)$. Note ainda que, para todo $x, y \in D(A)$,

$$
\langle(A-m I) x, y\rangle=\langle A x, y\rangle-\langle m x, y\rangle=\langle x, A y\rangle-\langle x, m y\rangle=\langle x,(A-m I) y\rangle
$$

e

$$
\langle(A-m I) x, x\rangle=\langle A x, x\rangle-m\langle x, x\rangle \geq m\|x\|^{2}-m\|x\|^{2}=0 .
$$

Fixamos $\lambda \in \Sigma_{\frac{\pi}{4}}(m)$ e definimos $\lambda_{1}=\lambda-m$. Dividiremos então em dois casos. No primeiro consideramos $\operatorname{Re} \lambda_{1}<0$ e, de 3.14 e 3.15 , obtemos

$$
\begin{aligned}
& \|(\lambda I-A) x\|^{2}=\left\|\left(\lambda_{1} I-(A-m I)\right) x\right\|^{2} \\
& \quad=\left|\lambda_{1}\right|^{2}\|x\|^{2}-2 \operatorname{Re} \lambda_{1}\langle(A-m I) x, x\rangle+\|(A-m I) x\|^{2}
\end{aligned}
$$




$$
\geq\left|\lambda_{1}\right|^{2}\|x\|^{2}
$$

Suponhamos agora, como um segundo caso, que $0 \leq \operatorname{Re} \lambda_{1} \leq\left|\operatorname{Im} \lambda_{1}\right|$ então, para $x \in D(A)$, temos

$$
\begin{aligned}
& \|(\lambda I-A) x\|^{2}=\left\|\left(\lambda_{1} I-(A-m I)\right) x\right\|^{2} \\
& \quad=\left|I m \lambda_{1}\right|^{2}\|x\|^{2}+\left\|\left(\operatorname{Re} \lambda_{1} I-(A-m I)\right) x\right\|^{2} \\
& \quad \geq\left|I m \lambda_{1}\right|^{2}\|x\|^{2} \geq \frac{\left|\lambda_{1}\right|^{2}}{2}\|x\|^{2},
\end{aligned}
$$

uma vez que

$$
\begin{aligned}
& \left\langle i \operatorname{Im} \lambda_{1} x,\left(\operatorname{Re} \lambda_{1} I-(A-m I)\right) x\right\rangle+\left\langle\left(\operatorname{Re} \lambda_{1} I-(A-m I)\right) x, i \operatorname{Im} \lambda_{1} x\right\rangle \\
& \quad=i \operatorname{Im} \lambda_{1} \operatorname{Re} \lambda_{1}\|x\|^{2}-i \operatorname{Re} \lambda_{1} \operatorname{Im} \lambda_{1}\|x\|^{2}-i \operatorname{Im} \lambda_{1}\langle(A-m I) x, x\rangle+i \operatorname{Im} \lambda_{1}\langle(A-m I) x, x\rangle=0 .
\end{aligned}
$$

Combinando os resultados acima, obtemos, para qualquer $\lambda \in \Sigma_{\frac{\pi}{4}}(m)$ e qualquer $x \in D(A)$,

$$
\|(\lambda I-A) x\| \geq \frac{|\lambda-m|}{\sqrt{2}}\|x\|
$$

De onde segue que, se $\lambda \in \Sigma_{\frac{\pi}{4}}(m)$ e $y \in H$ então $x=(\lambda I-A) y \in D(A)$ e

$$
\|(\lambda I-A) y\| \leq \frac{\sqrt{2}}{|\lambda-m|}\|y\|
$$

Portanto,

$$
\|(\lambda I-A)\| \leq \frac{\sqrt{2}}{|\lambda-m|}, \quad \forall \lambda \in \Sigma_{\frac{\pi}{4}}(m) .
$$

Logo $A$ é um operador setorial em $H$.

O próximo resultado, que será crucial para o estudo do sistema 2.30- 2.32, afirma que o produto cartesiano de operadores setoriais é também um operador setorial.

Proposição 4 Sejam $A: D(A) \subset X \longrightarrow X$ e $B: D(B) \subset Y \longrightarrow Y$ operadores setoriais nos espaços de Banach $X$ e $Y$, respectivamente. Então o operador

$$
A \times B: D(A) \times D(B) \longrightarrow X \times Y,
$$

dado por $(A \times B)(x, y)=(A x, B y)$ para todo $(x, y) \in D(A) \times D(B)$, é setorial em $X \times Y$.

Demonstração. Sendo $A$ e $B$ operadores lineares fechados e densamente definidos, então $A \times B$ também tem as mesmas propriedades. Além disso, existem contantes $a_{1}, M_{1}$ e $\sigma_{1}$ associadas ao operador $A$ tais que as condições da definição 25 são satisfeitas. Existem ainda constantes $a_{2}, M_{2}$ e $\sigma_{2}$ associadas ao operador $B$ de modo que as mesmas condições são satisfeitas, desta vez para o 
operador $B$. Uma vez que $\rho(A \times B)=\rho(A) \cap \rho(B)$, basta tomarmos

$$
a=\min a_{1}, a_{2} \text { e } \sigma=\max \sigma_{1}, \sigma_{2}
$$

e assim, $\Sigma_{\sigma}(a) \subset \rho(A \times B)$. Se $M=\max M_{1}, M_{2}$, então

$$
\left\|(\lambda I-A \times B)^{-1}\right\|=\left\|(\lambda I-A, \lambda I-B)^{-1}\right\|=\max \left\{\left\|(\lambda I+A)^{-1}\right\|,\left\|(\lambda I+B)^{-1}\right\|\right\} \leq \frac{M}{|\lambda-a|},
$$

para todo $\lambda \in \Sigma_{\sigma}(a)$. De onde segue que $A \times B$ é um operador setorial.

Devemos agora provar que um operador como o definido acima, de fato, gera um semigrupo analítico. O que na verdade se verifica, como veremos no teorema a seguir, é que se um operador $B$ é setorial, então $-B$ é gerador infinitesimal de um semigrupo analítico. É possivel ainda provar que a recíproca é verdadeira, ou seja, se $-B$ gera um semigrupo analítico então $B$ é um operador setorial [22]. Esta equivalência é muito importante para a resolução de problemas de valor inicial lineares ou não -, como veremos nas próximas seções.

Antes de apresentarmos o resultado que nos garante parte desta equivalência, faremos algumas observações que nos serão úteis para a demonstração.

1. Seja $A$ um operador setorial, que satisfaz 3.13 para constantes $a$ e $M$, com $\lambda$ em algum setor $\Sigma_{\sigma}(a)$. Tomando $\delta=\pi-\sigma$, obtemos que

$$
\left\|(\lambda I+A)^{-1}\right\| \leq \frac{M}{|\lambda+a|}, \quad \forall \lambda \in \Delta_{\delta}(-a) .
$$

De fato, sendo $\sigma+\delta=\pi$, obtemos de 3.12 que

$$
\Sigma_{\sigma}(a) \subset \rho(A) \Longleftrightarrow \Delta_{\delta}(-a) \subset \rho(-A) .
$$

Então se $\lambda \in \Delta_{\delta}(-a)$, verifica-se que $\lambda$ está no resolvente de $-A$ e, portanto, é possivel obter uma limitação para $(\lambda I+A)^{-1}$.

2. Podemos ainda obter

$$
\left\|(\mu I+B)^{-1}\right\| \leq \frac{M}{|\mu|}, \quad \forall \mu \in \Delta_{\delta}
$$

onde $B=A-a I$. De onde segue que, $A$ é um operador setorial se, e somente se, $B$ é setorial.

Observamos aqui que existe uma relação entre um semigrupo $T(t)$ em $\mathcal{L}(\mathcal{X})$ e o problema de Cauchy,

$$
\begin{aligned}
\frac{d}{d t} x(t) & =A x(t) \\
x(0) & =x_{0} .
\end{aligned}
$$


$T(t)$ é, na verdade, solução do problema ${ }^{12}$

$$
\begin{aligned}
\frac{d}{d t} T(t) & =A T(t) \\
T(0) & =I_{X} .
\end{aligned}
$$

Então, em geral, denota-se o semigrupo $T(t) \operatorname{como} e^{t A}$.

Teorema 17 Sejam $X$ um espaço de Banach e $A: D(A) \subset X \longrightarrow X$ um operador setorial. Sejam ainda $M \geq 1, a \in \mathbb{R}$ e $\sigma \in\left(0, \frac{\pi}{2}\right)$ constantes satisfazendo as condições da definição 25, para $o$ operador A. Então valem as seguintes afirmações

1. $-A$ é o gerador infinitesimal de um semigrupo analítico $T(t): t \geq 0 \subset \mathcal{L}(X)$, onde

$$
T(t)=\frac{1}{2 \pi i} \int_{\Gamma}(\lambda I+A)^{-1} e^{\lambda t} d \lambda
$$

onde $\Gamma=\Gamma(\theta)$ representa uma curva de $\infty e^{-i \theta}$ para $\infty e^{i \theta}$ contida no resolvente de $-A e$ $\theta \in\left(\frac{\pi}{2}, \pi-\sigma\right)$;

2. para qualquer $\epsilon \in(0, \theta)$, existe uma constante $C=C(\epsilon)$ tal que

$$
\|T(t)\| \leq C, \quad\|A T(t)\| \leq \frac{C}{|t|}, \quad t \in \Delta_{\theta-\epsilon}
$$

3. $A T(t), \frac{d T(t)}{d t}$ são operadores limitados para $t \in \Delta_{\theta} e$

$$
\frac{d T(t) x}{d t}=-A T(t) x, \quad x \in X
$$

Demonstração. De acordo com as observações 1 e 2, podemos supor, sem perda de generalidade, que $a=0$ e $\left\|(\lambda+A)^{-1}\right\| \leq \frac{M}{|\lambda|}$, para $\lambda \in \Delta_{\delta}$. Considere a seguinte definição

$$
e^{-t A} \doteq \frac{1}{2 \pi i} \int_{\Gamma} e^{\lambda t}(\lambda I+A)^{-1} d \lambda
$$

A integral está bem definida em $\Gamma_{n}=\Gamma \cap\{\lambda:|\lambda<n|\}$, pois o integrando é uma função de $\lambda \in \Gamma$ no conjunto das funções lineares e limitadas em $X$. Basta então considerar 3.21 como uma integral imprópria. Observamos ainda que a integral acima converge absolutamente. Nosso próximo passo é provar que ela, de fato, satisfaz as propriedades de semigrupo.

O teorema de Cauchy nos garante que

$$
\frac{1}{2 \pi i} \int_{\Gamma} e^{\lambda t}(\lambda I+A)^{-1} d \lambda=\frac{1}{2 \pi i} \int_{\Gamma^{\prime}} e^{\lambda t}(\lambda I+A)^{-1} d \lambda
$$

\footnotetext{
${ }^{12} \mathrm{O}$ operador $T(t)$ é, por essa razão, também conhecido como operador solução.
} 
para o caso em que, por exemplo, $\Gamma^{\prime}$ é definido como sendo a curva obtida de uma translação de cada ponto $\lambda \in \Gamma$ para a direita por uma distância fixa e positiva. Dados $t, s>0$, podemos então afirmar que

$$
e^{-t A}=\frac{1}{2 \pi i} \int_{\Gamma} e^{\lambda t}(\lambda I+A)^{-1} d \lambda
$$

$\mathrm{e}$

$$
e^{-s A}=\frac{1}{2 \pi i} \int_{\Gamma^{\prime}} e^{\mu s}(\mu I+A)^{-1} d \mu .
$$

Obtemos assim, pelo Teorema de Fubini, que

$$
e^{-t A} e^{-s A}=\frac{1}{(2 \pi i)^{2}} \int_{\Gamma} \int_{\Gamma^{\prime}} e^{\lambda t+\mu s}(\lambda I+A)^{-1}(\mu I+A)^{-1} d \lambda d \mu .
$$

Sendo $\lambda \neq \mu$, podemos usar a seguinte fórmula do resolvente

$$
(\lambda+A)^{-1}-(\mu+A)^{-1}=(\mu-\lambda)(\lambda+A)^{-1}(\mu+A)^{-1},
$$

que pode ser obtida multiplicando ambos os lados por $(\lambda+A)^{-1}(\mu+A)^{-1}$. Então,

$$
e^{-t A} e^{-s A}=\frac{1}{(2 \pi i)^{2}} \int_{\Gamma} \int_{\Gamma^{\prime}} e^{\lambda t+\mu s}(\mu-\lambda)^{-1}\left\{(\lambda I+A)^{-1}-(\mu I+A)^{-1}\right\} d \lambda d \mu .
$$

Como a curva $\Gamma^{\prime}$ está a direita da curva $\Gamma$, obtemos, também pelo Teorema de Cauchy,

$$
\int_{\Gamma} e^{\lambda t}(\mu-\lambda)^{-1} d \lambda=0
$$

e

$$
\int_{\Gamma^{\prime}} e^{\mu t}(\mu-\lambda)^{-1} d \mu=2 \pi i e^{\lambda s}
$$

pois $\mu$ não pertence ao interior da curva $\Gamma$, enquanto $\lambda$ está no interior de $\Gamma^{\prime}$. É possivel assim obter, pelo Teorema de Fubini,

$$
e^{-t A} e^{-s A}=\frac{1}{2 \pi i} \int_{\Gamma} e^{\lambda(t+s)}(\lambda I+A)^{-1} d \lambda=e^{-(t+s) A}
$$

Observamos que, se $\epsilon \in\left(0, \theta-\frac{\pi}{2}\right)$, então a integral converge uniformemente em qualquer compacto de $\Delta_{\epsilon}$. Apenas por uma questão de conveniência, provaremos primeiro as afirmações 2 e 3 e em seguida que 3.21 define um semigrupo fortemente contínuo.

Se $\lambda \in \Gamma$, então $\frac{\lambda}{|t|}$ pertence ao setor $\Delta_{\delta}$, para $t \neq 0$, e portanto, vale que

$$
\left\|\left(\frac{\lambda}{|t|} I+A\right)^{-1}\right\| \leq \frac{C}{\left|\frac{\lambda}{t}\right|} \leq \frac{C|t|}{|\lambda|},
$$


onde $C=C(\epsilon)$ é constante, para cada $\epsilon$. Substituindo $\lambda$, em 3.21, por $\frac{\mu}{|t|}$, obtemos

$$
e^{-t A}=\frac{1}{2 \pi i} \int_{\Gamma^{\prime \prime}} e^{\mu \rho}\left(\frac{\mu}{|t|} I+A\right)^{-1} \frac{d \mu}{|t|}=\frac{1}{2 \pi i} \int_{\Gamma} e^{\mu \rho}\left(\frac{\mu}{|t|} I+A\right)^{-1} \frac{d \mu}{|t|},
$$

onde $\Gamma^{\prime \prime}=|t| \Gamma$ e $\rho=\arg t$. Observamos que, mais uma vez, usamos o Teorema de Cauchy quando afirmamos que a integral não se altera após a substituição de $\Gamma^{\prime \prime}$ por $\Gamma$.

Obtemos assim, de 3.22 e 3.23 , a desigualdade

$$
\left\|e^{-t A}\right\| \leq \frac{1}{2 \pi} \int_{\Gamma}\left\|e^{\mu \rho}\left(\frac{\mu}{|t|} I+A\right)^{-1} \frac{d \mu}{|t|}\right\| \leq \frac{C}{2 \pi} \int_{\Gamma}\left|e^{\mu \rho}\right| \frac{|t|}{|\mu|} \frac{|d \mu|}{|t|} \leq C .
$$

Além disso,

$$
\begin{aligned}
A e^{-t A} & =\frac{1}{2 \pi i} \int_{\Gamma} e^{\mu \rho} A\left(\frac{\mu}{|t|} I+A\right)^{-1} \frac{d \mu}{|t|} \\
& =\frac{1}{2 \pi i} \int_{\Gamma} e^{\mu \rho}\left\{\left(A+\frac{\mu}{|t|} I\right)-\frac{\mu}{|t|} I\right\}\left(\frac{\mu}{|t|} I+A\right)^{-1} \frac{d \mu}{|t|} \\
& =\frac{1}{2 \pi i} \int_{\Gamma} e^{\mu \rho} \frac{d \mu}{|t|}-\frac{1}{2 \pi i} \int_{\Gamma} e^{\mu \rho} \frac{\mu}{|t|}\left(\frac{\mu}{|t|} I+A\right)^{-1} \frac{d \mu}{|t|} \\
& =-\frac{1}{2 \pi i} \int_{\Gamma} e^{\mu \rho} \frac{\mu}{|t|}\left(\frac{\mu}{|t|} I+A\right)^{-1} \frac{d \mu}{|t|}
\end{aligned}
$$

e, portanto,

$$
\left\|A e^{-t A}\right\| \leq \frac{C}{2 \pi} \int_{\Gamma}\left|e^{\mu \rho}\right| \frac{|d \mu|}{|t|} \leq \frac{C}{|t|} .
$$

Observamos que as limitações obtidas acima são válidas para o caso em que $|\rho|<\theta-\epsilon$.

Obtemos de 3.21 que $e^{-t A}$ é diferenciável em $t$ e ainda que, para $x \in D(A)$,

$$
\begin{aligned}
\frac{d}{d t} e^{-t A} x+A e^{-A t} x=\frac{1}{2 \pi i} \int_{\Gamma} e^{\lambda t}\left\{\lambda(\lambda I+A)^{-1}+A(\lambda I+A)^{-1}\right\} d \lambda \\
\quad=\frac{1}{2 \pi i} \int_{\Gamma} e^{\lambda t}\left\{I-A(\lambda I+A)^{-1}+A(\lambda I+A)^{-1}\right\} d \lambda \\
\quad=\frac{1}{2 \pi i} \int_{\Gamma} e^{\lambda t} d \lambda \\
\quad=0 .
\end{aligned}
$$

Sendo $A$ um operador fechado, obtemos ainda que

$$
\frac{d}{d t} e^{-t A} x=A e^{-A t} x, \quad \forall x \in X
$$

Sejam $x \in D(A)$ e $t \in \Delta_{\theta-\epsilon}$, então

$$
e^{-t A} x-x=\frac{1}{2 \pi i} \int_{\Gamma} e^{\lambda t}\left\{(\lambda I+A)^{-1}-\lambda^{-1}\right\} x d \lambda=-\frac{1}{2 \pi i} \int_{\Gamma} \lambda^{-1} e^{\lambda t} A(\lambda I+A)^{-1} x d \lambda .
$$


Segue assim que,

$$
\lim _{t \rightarrow 0} e^{-t A} x-x=-\frac{1}{2 \pi i} \int_{\Gamma} \lambda^{-1} A(\lambda I+A)^{-1} x d \lambda=0 .
$$

A conclusão é a mesma para o caso em que $x \in X$ usando o fato de que $\left\|e^{-t A}\right\| \leq C$, se $t \in \Delta_{\theta-\epsilon}$.

Concluímos então que, de fato, $\left\{e^{-t A}\right\}_{t \geq 0}$ é um semigrupo analítico em $\Delta_{\theta-\epsilon}$. Observamos ainda que o Teorema de Cauchy nos garante que esta conclusão não depende da curva $\Gamma$, ou seja, não depende de $\theta$. Resta-nos provar que $-A$ é o gerador infinitesimal de $e^{-t A}$.

Tomemos então $x \in D(A)$. Segue pelo Teorema Fundamental do Cálculo, que

$$
\lim _{t \rightarrow 0} \frac{e^{-t A}-x}{t}=\lim _{t \rightarrow 0} \frac{1}{t} \int_{t}^{0} e^{-s A} A x d s=-A x .
$$

O que, por sua vez, nos leva a concluir que o domínio do gerador $B$ de $e^{-t A}$ contém o domínio de $A$ e $B \mid D(A)=-A$. É suficiente então provarmos que os domínios de $A$ e de $B$ coincidem.

Já que $D(A) \subset D(B)$, nos resta provar que $D(B) \subset D(A)$, onde $D(B)$ é o domínio do operador $B$. Defina então, para cada $\lambda \geq 0$,

$$
P_{\lambda} x=\int_{0}^{\infty} e^{-\lambda t} e^{-t A} d t
$$

Como $\left\|e^{-t A}\right\| \leq C$, a integral está bem definida pois converge absolutamente, se $x \in X$. Se provarmos que

(i) $D(B) \subset R\left(P_{\lambda}\right)$ e

(ii) $R\left(P_{\lambda}\right) \subset D(A)$, onde $R\left(P_{\lambda}\right)$ é a imagem de $X$ por $V$, obteremos o resultado.

Seja $x \in X$. Afirmamos que $P_{\lambda} x \in D(A)$. De fato,

$$
\begin{array}{rl}
e^{-s A} & P \lambda x-P_{\lambda} x=\frac{e^{-s A} \int_{0}^{\infty} e^{-\lambda t} e^{-t A} d t-\int_{0}^{\infty} e^{-\lambda t} e^{-t A} d t}{s} \\
= & \frac{1}{s} \int_{0}^{\infty} e^{-\lambda t} e^{(t+s) A} x d t-\frac{1}{s} \int_{0}^{\infty} e^{-\lambda t} e^{-t A} x d t \\
= & \frac{e^{\lambda s}-1}{s} \int_{0}^{\infty} e^{-\lambda t} e^{-t A} x d t-\frac{e^{\lambda s}}{s} \int_{0}^{s} e^{-\lambda t} e^{-t A} x d t,
\end{array}
$$

e, portanto,

$$
\lim _{s \rightarrow 0} e^{-s A} P_{\lambda} x-P_{\lambda} x=\lambda P_{\lambda} x-x .
$$

De onde segue $(i i)$. Obtemos, ainda do limite acima, que

$$
(\lambda I-B) P \lambda x=x .
$$

Além disso, se $x \in D(B)$ então $e^{-t A} \in D(B)$, para todo $t \geq 0$, e

$$
B(P \lambda x)=B \int_{0}^{\infty} e^{-\lambda t} e^{-t A} x d t=\int_{0}^{\infty} e^{-\lambda t} e^{-t A} B x d t=P_{\lambda}(B x) .
$$


Logo, de 3.24 e da igualdade acima, obtemos

$$
P_{\lambda}(\lambda I-B) x=x
$$

de onde segue $(i)$.

\subsubsection{Potências fracionárias de operadores}

Dedicaremos esta subseção ao estudo das potências fracionárias de operadores setoriais. Veremos na última seção deste capítulo que este estudo desempenhará um papel fundamental na teoria de existência de soluções para problemas de Cauchy semilineares do tipo parabólico. Além disso, nos permitirá analisar o comportamento assintótico das soluções de tais problemas.

Uma vez que as definições e resultados desta subseção envolvem o conceito da função Gama, introduziremos inicialmente a seguinte

Definição 26 Para cada $z \in \mathbb{C}$ a função Gama em z é definida por

$$
\Gamma(z)=\int_{0}^{\infty} t^{z-1} e^{-t} d t
$$

A função Gama pode ser vista como uma extensão da função fatorial, para o plano complexo. Além disso, $\Gamma$ satisfaz a seguinte propriedade, conhecida como Fórmula de Reflexão de Euler,

$$
\Gamma(1-z) \Gamma(z)=\frac{\pi}{\sin \pi z} .
$$

Definição 27 Suponha que A seja um operador setorial e Re $\lambda>0$, para todo $\lambda \in \sigma(A)$. Então, para qualquer $\alpha>0$, definimos

$$
A^{-\alpha}=\frac{1}{\Gamma(\alpha)} \int_{0}^{\infty} t^{\alpha-1} e^{-A t} d t
$$

O próximo teorema nos fornece uma fórmula alternativa a 3.25 , mas que pode ser usada como definição de potência fracionária para casos mais gerais. Pode ser usada, por exemplo, para o caso em que operador $A$ não é setorial. Apesar disso nos restringimos ao contexto da definição 3.25.

Teorema 18 Seja $A$ um operador setorial em $X$ tal que Re $\lambda>0$, para todo $\lambda \in \sigma(A)$. Então, para $\alpha \in(0,1)$,

$$
A^{-\alpha}=\frac{\sin \pi \alpha}{\pi} \int_{0}^{\infty} \lambda^{-\alpha}(\lambda I+A)^{-1} d \lambda .
$$

Demonstração. De acordo com o teorema 9, para todo $x \in X,(\lambda+A)^{-1}=\int_{0}^{\infty} e^{-A t} e^{-\lambda t} d t$, para $\lambda>0$. Então,

$$
\begin{aligned}
\int_{0}^{\infty} & \lambda^{-\alpha}(\lambda+A)^{-1} d \lambda=\int_{0}^{\infty} \lambda^{-\alpha}\left(\int_{0}^{\infty} e^{-A t} e^{-\lambda t} d t\right) d \lambda \\
& =\int_{0}^{\infty} e^{-A t}\left(\int_{0}^{\infty} \lambda^{-\alpha} e^{-\lambda t} d \lambda\right) d t
\end{aligned}
$$




$$
\begin{aligned}
& =\int_{0}^{\infty} e^{-A t} t^{\alpha-1} \Gamma(1-\alpha) d t \\
& =\frac{\sin \pi \alpha}{\pi} A^{-\alpha},
\end{aligned}
$$

uma vez que $\Gamma(\alpha) \Gamma(1-\alpha)=\frac{\sin \pi \alpha}{\pi}$.

Seguem abaixo alguns resultados acerca das propriedades das potências fracionárias de um operador setorial $A$.

Lema 11 Seja $A$ é um operador setorial em $X$ tal que Re $\lambda>0$, para todo $\lambda \in \sigma(A)$. Então, $A^{-\alpha} \in \mathcal{L}(X)$ e $A^{-\alpha} A^{-\beta}=A^{-(\alpha+\beta)}$, para todo $\alpha \geq 0$ e $\beta \geq 0$.

Demonstração. Vamos verificar que $A^{-\alpha} \in \mathcal{L}(X)$ exibindo uma constante $C$ que limita o operador $A^{-\alpha}$. De acordo com o teorema 21

$$
A^{-\alpha}=\frac{\sin \pi \alpha}{\pi} \int_{0}^{\infty} \lambda^{-\alpha}(\lambda+A)^{-1} d \lambda
$$

e, portanto,

$$
\left\|A^{-\alpha}\right\| \leq\left\|\frac{\sin \pi \alpha}{\pi} \int_{0}^{1} \lambda^{-\alpha}(\lambda+A)^{-1} d \lambda\right\|+\left\|\frac{\sin \pi \alpha}{\pi} \int_{1}^{\infty} \lambda^{-1-\alpha} \lambda(\lambda+A)^{-1} d \lambda\right\| .
$$

Sejam $C_{1}$ e $C_{2}$ constantes positivas tais que $\left\|(\lambda I+A)^{-1}\right\| \leq C_{1}$, se $t \in[0,1]$, e $\left\|\lambda(\lambda I+A)^{-1}\right\| \leq C_{2}$. A existência de tais constantes é garantida pelo teorema 21. Obtemos então que,

$$
\left\|A^{-\alpha}\right\| \leq\left|\frac{\sin \pi(1-\alpha)}{\pi(1-\alpha)}\right| C_{1}+\left|\frac{\sin \pi \alpha)}{\pi \alpha)}\right| C_{2} \leq C .
$$

Nosso próximo passo é provar que $A^{-\alpha} A^{-\beta}=A^{-(\alpha+\beta)}$, se $\alpha>0$ e $\beta>0$. De fato,

$$
\begin{aligned}
A^{-\alpha} & A^{-\beta}=\frac{1}{\Gamma(\alpha) \Gamma(\beta)} \int_{0}^{\infty} \int_{0}^{\infty} t^{\alpha-1} s^{\beta-1} T(t) T(s) d t d s \\
& =\frac{1}{\Gamma(\alpha) \Gamma(\beta)} \int_{0}^{\infty} t^{\alpha-1} \int_{0}^{\infty}(u-t)^{\beta-1} T(u) d u d t \\
& =\frac{1}{\Gamma(\alpha) \Gamma(\beta)} \int_{0}^{\infty} \int_{0}^{u} t^{\alpha-1}(u-t)^{\beta-1} d t T(u) d u \\
& =\frac{1}{\Gamma(\alpha+\beta)} \int_{0}^{1} z^{\alpha-1}(1-z)^{\beta-1} d z \cdot \int_{0}^{\infty} u^{\alpha+\beta-1} T(u) d u \\
& =A^{-(\alpha+\beta)} .
\end{aligned}
$$

Lema 12 Se $A$ é um operador setorial em $X$, então $A^{-\alpha}$ é injetora, para todo $\alpha>0$.

Demonstração. Se $\alpha=1$, a afirmação é válida. O que implica que $A^{-n}$ é injetora, para todo inteiro $n \geq 1$. Seja $x \in X$ tal que $A^{-\alpha} x=0$, para algum $\alpha>0$. Tomando algum inteiro $n$ com 
$n>\alpha$, temos que $A^{-n} x=A^{-n+\alpha} A^{-\alpha} x=0$, de onde obtemos que $x=0$. Logo $A^{-\alpha}$ é injetora, para todo $\alpha>0$.

Estendemos a seguir a definição 27 para o caso em que $\alpha \leq 0$.

Definição 28 Seja A um operador setorial tal que Re $\lambda>0$, para todo $\lambda \in \sigma(A)$. Definimos, para todo $\alpha>0$,

$$
A^{\alpha}=\left(A^{-\alpha}\right)^{-1}
$$

com $D\left(A^{\alpha}\right)=R\left(A^{-\alpha}\right)$. Se $\alpha=0$, definimos $A^{\alpha}=I$.

Teorema 19 Seja $A^{\alpha}$ como na definição acima. Então são válidas as seguintes afirmações:

1. se $\alpha>0, A^{\alpha}$ é um operador fechado;

2. se $\alpha \geq \beta>0$ então $D\left(A^{\alpha}\right) \subset D(\beta)$;

3. $D\left(A^{\alpha}\right)$ é denso em $X$, para todo $\alpha \geq 0$;

4. se $\alpha$ e $\beta$ são reais então $A^{\alpha+\beta} x=A^{\alpha} \cdot A^{\beta} x$, para todo $x \in D\left(A^{\gamma}\right)$, onde $\gamma=\max (\alpha, \beta, \alpha+\beta)$.

\section{Demonstração.}

1. Se $\alpha>0, A^{\alpha}$ é invertível e, portanto, $0 \in \rho\left(A^{\alpha}\right)$. O que implica que $A^{\alpha}$ é fechado.

2. Se $\alpha \geq \beta$, é possível obter, aplicando o lema $12, A^{-\alpha}=A^{-\beta} \cdot A^{-(\alpha-\beta)}$. De onde segue que $D\left(A^{\alpha}\right) \subset D(\beta)$.

3. De acordo com item 2 do teorema $9, \overline{D\left(A^{n}\right)}=X$ para $n=1,2,3, \cdots$. Além disso, pelo item anterior, temos que $D\left(A^{n}\right) \subset D\left(A^{\alpha}\right)$ para $\alpha \leq n$. De onde segue a afirmação 3 .

4. Para verificarmos a última afirmação devemos dividir em dois casos. No primeiro supomos $\alpha>0$ e $\beta>0$, já no segundo consideramos $\alpha>0$ e $\beta \leq 0$. São os únicos a ser considerados, uma vez que um terceiro caso seria aquele proposto no lema 12. Verifiquemos então o primeiro caso. Se $x \in D\left(A^{\alpha} A^{\beta}\right)$ então $x \in D\left(A^{\beta}\right)$ e $A^{\beta} x \in D\left(A^{\alpha}\right)$. Logo

$$
y=A^{\alpha} A^{\beta} x \Rightarrow A^{-\alpha} y=A^{\beta} x \Rightarrow x=A^{-\beta} A^{-\alpha} y=A^{-(\alpha+\beta)} y
$$

e, portanto, $x \in D\left(A^{\alpha+\beta}\right)$ e $A^{\alpha+\beta} x=y=A^{\alpha} A^{\beta} x$. Da mesma forma, se $x \in D\left(A^{\alpha+\beta}\right)$ é possível concluir que $x \in D\left(A^{\alpha} A^{\beta}\right)$ e $A^{\alpha+\beta} x=A^{\alpha} A^{\beta} x$. O segundo caso também é uma conseqüência da definição de $A^{\alpha}$ e do lema 12 .

O teorema a seguir nos fornece uma fórmula explícita para $A^{\alpha} x$, com $\alpha \in(0,1)$ e $x \in D(A) \subset$ $D\left(A^{\alpha}\right)$, de maneira semelhante a que obtemos para $A^{-\alpha} x$ no teorema 18. 
Teorema 20 Seja $A$ um operador setorial em $X$ tal que Re $\lambda>0$, para todo $\lambda \in \sigma(A)$. Então, para $\alpha \in(0,1)$ e $x \in D\left(A^{\alpha}\right)$,

$$
A^{\alpha} x=\frac{\sin \pi \alpha}{\pi} \int_{0}^{\infty} \lambda^{\alpha-1} A(\lambda I+A)^{-1} x d \lambda
$$

Demonstração. Se $0<\alpha<1$, então $0<1-\alpha<1$. Obtemos assim, pelo teorema 18,

$$
A^{\alpha-1} x=\frac{\sin \pi \alpha}{\pi} \int_{0}^{\infty} \lambda^{\alpha-1}(\lambda I+A)^{-1} x d \lambda
$$

para $x \in D\left(A^{\alpha}\right)$. Aplicando o operador $A$ de ambos os lados da igualdade acima, temos que

$$
A^{\alpha} x=\frac{\sin \pi \alpha}{\pi} \int_{0}^{\infty} \lambda^{\alpha-1} A(\lambda I+A)^{-1} x d \lambda
$$

Sendo $x$ um elemento de $D\left(A^{\alpha}\right)$, então $A^{\alpha-1} x \in D(A)$ e, portanto, o primeiro lado da igualdade 3.28 faz sentido. Além disso, o integrando em 3.27 pertence ao domínio de $A$ para todo $t>0$ e a última integral está bem definida, uma vez que $A(\lambda I+A)^{-1} x d \lambda$ é uniformemente limitado numa vizinhança de $t=0$ e $\left\|\lambda^{\alpha-1} A(\lambda I+A)^{-1} x\right\| \leq \lambda^{\alpha-2} M\|A x\|$, onde $M$ é a constante dada pelo teorema 5 . Logo $\lambda^{\alpha-1} A(\lambda I+A)^{-1} x$ é integrável em $[0, \infty[$.

Teorema 21 Sejam A um operador setorial em $X$ e $\alpha \in[0,1]$. Então existe uma constante $C>0$, que só depende de $A$, tal que

$$
\left\|A^{\alpha} x\right\| \leq C\|A x\|^{\alpha}\|x\|^{1-\alpha}
$$

para todo $\alpha \in[0,1]$ e $x \in D(A)$.

Demonstração. O resultado segue imediatamente se $\alpha=0$ ou $\alpha=1$. Suponha então $\alpha \in(0,1)$. De acordo com o teorema anterior

$$
A^{\alpha} x=\frac{\sin \pi \alpha}{\pi} \int_{0}^{\infty} \lambda^{\alpha-1} A(\lambda I+A)^{-1} x d \lambda
$$

de onde obtemos a seguinte limitação

$$
\begin{gathered}
\left\|A^{\alpha} x\right\| \leq \frac{\sin \pi \alpha}{\pi}\left[\int_{0}^{\rho} \lambda^{\alpha-1}(M+1)\|x\| d \lambda+\int_{\rho}^{\infty} \lambda^{\alpha-2} M\|A x\| d \lambda\right] \\
\leq \frac{\sin \pi \alpha}{\pi}(M+1)\left(\frac{\rho^{\alpha}}{\alpha}\|x\|+\frac{\rho^{\alpha-1}}{1-\alpha}\|A x\|\right),
\end{gathered}
$$

onde $\rho$ é uma constante positiva qualquer e $M$ é a constante dada pelo teorema 5 . Tomando $\rho=\frac{\|A x\|}{\|x\|}$, obtemos

$$
\left\|A^{\alpha} x\right\| \leq \frac{\sin \pi \alpha}{\pi}(M+1)\left(\frac{\|A x\|^{\alpha}}{\|x\|^{\alpha-1} \alpha}+\frac{\|A x\|^{\alpha}}{\|x\|^{\alpha-1}(1-\alpha)}\right)
$$




$$
\leq \frac{\sin \pi \alpha}{\pi}(M+1)\left(\frac{1}{\alpha}+\frac{1}{1-\alpha}\right)\|A x\|^{\alpha}\|x\|^{1-\alpha}
$$

Basta definirmos $C=\sup _{\alpha \in(0,1)}\left\{\frac{\sin \pi \alpha}{\pi}(M+1)\left(\frac{1}{\alpha}+\frac{1}{1-\alpha}\right)\right\}$ e então concluiremos o resultado.

Teorema 22 Sejam $A$ e $B$ operadores setoriais em $X$ tais que $D(A)=D(B)$ e Re $\lambda>0$ para todo $\lambda \in \sigma(A) \cup \sigma(B)$. Se além disso $(A-B) A^{-\alpha} \in \mathcal{L}(X)$, para algum $\alpha \in[0,1)$, então $A^{\beta} B^{-\beta} e$ $B^{\beta} A^{-\beta}$ são limitados em $X$, para todo $\beta \in[0,1]$.

Demonstração. Pelo teorema anterior, $\left\|A^{\beta}(\lambda I+A)^{-1}\right\| \leq C|\lambda|^{\beta-1}$ em $\{\lambda \in \mathbb{C}:|\pi-\arg \lambda| \geq \theta\}$, para $\beta \in[0,1]$, alguma constante positiva $C$ e $\theta<\frac{\pi}{2}$. Para os casos $\beta=0$ e $\beta=1$, o resultado segue diretamente. Suponhamos então que $\beta \in(0,1)$, então

$$
B^{-\beta}-A^{-\beta}=\frac{\sin \pi \beta}{\pi} \int_{0}^{\infty} \lambda^{-\beta}(\lambda I+B)^{-1}(A-B)(\lambda I+A)^{-1} d \lambda .
$$

De onde é possível obter que $B^{\beta} A^{-\beta}$ é limitado.

Uma vez que

$$
\left[I+A^{\alpha}(\lambda I+A)^{-1}(B-A) A^{-\alpha}\right] A^{\alpha}(\lambda I+B)^{-1}=A^{\alpha}(\lambda I+A)^{-1}
$$

obtemos $\left\|A^{\alpha}(\lambda I+B)^{-1}\right\|=O\left(|\lambda|^{\alpha-1}\right)$, se $|\lambda| \rightarrow \infty$. Substituindo $A$ por $B$ na identidade integral acima, obtemos que $A^{\beta} B^{-\beta}$ também é um operador limitado.

Definição 29 Seja A um operador setorial em um espaço de Banach $X$. Para cada $\alpha \geq 0$, definese o espaço

$$
X^{\alpha}=D\left((a I+A)^{\alpha}\right),
$$

para algum $a \in \mathbb{R}$, com a norma do gráfico dada por

$$
\|x\|_{\alpha}=\left\|(a I+A)^{\alpha}\right\|,
$$

para cada $x \in X^{\alpha}$.

Afirmamos que $X^{\alpha}$ é um espaço de Banach com a norma $\|\cdot\|_{\alpha}$, uma vez que $0 \in \sigma\left((a I+A)^{\alpha}\right)$. Afirmamos ainda que o espaço $X^{\alpha}$ não depende da escolha de $a$ e que valores distintos de $a$ fornecem normas equivalentes em $X^{\alpha}$. Tais afirmações serão verificadas nos dois próximos resultados.

Teorema 23 Sejam $A$ e $B$ operadores setoriais em $X$ tais que $D(A)=D(B)$ e Re $\lambda>0$ para todo $\lambda \in \sigma(A) \cup \sigma(B)$. Se além disso

$$
\|(A-B) x\| \leq C\|A x\|^{\alpha}\|x\|^{1-\alpha}, \quad \forall x \in D(A),
$$

para algum $\alpha \in[0,1)$ e alguma constante $C$ positiva, então $D\left(A^{\beta}\right)=D\left(B^{\beta}\right)$, para todo $\beta \in[0,1]$. 
Esta demonstração não será apresentada aqui. O leitor é referido à [33].

Proposição 5 Sejam $A$ um operador setorial em $X, A_{1}=a I+A$ e $A_{2}=b I+A$, para $\alpha \in[0,1]$ e constantes distintas a e b. Então $D\left(A_{1}^{\alpha}\right)=D\left(A_{2}^{\alpha}\right)$ e as normas geradas por $A_{1}$ e $A_{2}$, como na definição 29, são equivalentes.

Demonstração. Aplicando o teorema anterior para $A_{1}$ e $A_{2}$, concluimos que $D\left(A_{1}^{\alpha}\right)=D\left(A_{2}^{\alpha}\right)$, para $\alpha \in[0,1]$. Sejam $\|\cdot\|_{\alpha_{1}}$ e $\|\cdot\|_{\alpha_{2}}$ as normas geradas por $A_{1}$ e $A_{2}$, respectivamente. Ou seja, para cada $x \in D\left(A_{1}^{\alpha}\right)=D\left(A_{2}\right)^{\alpha}$, tem-se $\|x\|_{\alpha_{1}}=\left\|A_{1}^{\alpha} x\right\|$ e $\|x\|_{\alpha_{2}}=\left\|A_{2}^{\alpha} x\right\|$. Nosso objetivo é mostrar que existem constantes $K_{1}$ e $K_{2}$ tais que

$$
K_{1}\|x\|_{\alpha_{1}} \leq\|x\|_{\alpha_{2}} \leq K_{2}\|x\|_{\alpha_{1}}
$$

Uma vez que, $\left(A_{1}-A_{2}\right) A_{1}^{\alpha}=(a-b) A_{1}^{\alpha}$, podemos aplicar o teorema 23 e concluir que

$$
\left\|A_{1}^{\alpha} A_{2}^{-\alpha}\right\| \leq K_{1} \quad \text { e }\left\|A_{2}^{\alpha} A_{1}^{-\alpha}\right\| \leq K_{2}
$$

para constantes $K_{1}$ e $K_{2}$. De onde segue que,

$$
K_{1}\left\|A_{1}^{\alpha} x\right\| \leq\left\|A_{2}^{\alpha} x\right\| \leq K_{2}\left\|A_{1}^{\alpha} x\right\|
$$

como queríamos demonstrar.

\subsection{Problemas de Cauchy lineares homogêneos e não homogêneos}

Sejam $X$ um espaço de Banach e $A: D(A) \subset X \longrightarrow X$ um operador linear densamente definido. Dado $u_{0} \in X$, o problema de Cauchy linear abstrato para $A$, com $u_{0}$ como dado inicial, é encontrar uma solução do seguinte problema

$$
\begin{array}{r}
u_{t}=A u(t), \quad \forall t>0 \\
u(0)=u_{0} .
\end{array}
$$

Mais precisamente, queremos saber em que condições o problema acima está bem-posto. O que significa dizer, como vimos no capítulo anterior, que o problema tem uma solução, ela é única e ainda depende continuamente do dado inicial.

A linearidade do problema 3.29 torna os argumentos acerca dos aspectos da sua boa colocação um pouco mais simples. Devido a tais facilidades discutiremos inicialmente os casos lineares homogêneo e não homogêneo, e na próxima seção passaremos ao caso semilinear ${ }^{13}$, que coincide com o problema de Cauchy de reação-difusão apresentado na subseção 2.1.1.

Antes de iniciarmos essa primeira discussão, precisamos esclarecer o que entendemos por solução de 3.29. Apresentamos então a seguinte

\footnotetext{
${ }^{13}$ Como referências adicionais àquelas utilizadas aqui e citadas na introdução, sugerimos [3] e [2]
} 
Definição 30 Dizemos que uma função $u:[0, \infty) \longrightarrow \mathbb{R}$ é uma solução clássica de 3.29 se u é contínua em $[0, \infty)$, continuamente diferenciavel, $u(t) \in D(A)$ para $t>0$ e u satisfaz 3.29.

Como já vimos na seção anterior, se $A$ for gerador de um semigrupo fortemente contínuo $T(t)$, então $u(t)=T(t) x$ é uma solução de 3.29 , ainda que $u$, assim definida, não seja continuamente diferenciável. Sendo assim, se faz necessária uma outra noção de solução do problema de Cauchy linear abstrato para $A$.

Definição 31 Se A é gerador de um semigrupo fortemente contínuo $T(t)$, dizemos que a função $u \in C((0, \infty) ; X)$ dada por

$$
u(t)=T(t) u_{0}, \quad \forall t \in(0, \infty),
$$

é uma solução fraca de $3.29 \mathrm{em} \mathbb{R}^{+}$.

O próximo resultado diz que se a condição inicial $u_{0}$ pertence ao domínio de $A$ e $A$ gera um semigrupo fortemente contínuo $T(t)$ então existe uma única solução fraca de 3.29 e ela é também uma solução clássica. Usaremos na próxima demonstração e na subseção seguinte o conceito de norma do gráfico. Portanto, segue inicialmente a

Definição 32 Sejam $X$ um espaço de Banach e $A: D(A) \subset X \longrightarrow X$ um operador linear, fechado e densamente definido. Dizemos que $\|\cdot\|_{A}$ é a norma do gráfico em $D(A)$, onde, para cada $x \in D(A)$,

$$
\|x\|_{A}=\|x\|+\|A x\| .
$$

Observamos que, se $A$ for invertível então a norma acima é equivalente a norma $\|x\|_{1}=\|A x\|$.

Teorema 24 Seja $A$ um operador linear densamente definido em um espaço de Banach $X$ tal que $\rho(A)$ é não vazio. Então o problema 3.29, com $u_{0} \in D(A)$, está bem posto se, e somente se, A gera um semigrupo fortemente contínuo em $X$.

Demonstração. Suponhamos a princípio que $A$ gera um semigrupo fortemente contínuo $T(t)$. Uma vez que $u_{0} \in D(A)$, podemos afirmar, pela definição que, a função $u:[0, \infty) \longrightarrow X$ dada por $u(t)=T(t) u_{0}$ é uma solução de 3.29. Além disso, $u \in C^{1}([0, \infty) ; X)$ e $\operatorname{Im}(u) \subset D(A)$. Nos resta então checar a unicidade de solução para 3.29. Suponha que a função $v$ seja uma outra solução e escolha $t$ e $s$ em $[0, \infty)$ de modo que $s \leq t$, então

$$
\frac{d}{d s}[T(t-s) v(s)]=T(t-s) A v(s)-A T(t-s) v(s)=T(t-s) A v(s)-T(t-s) A v(s)=0 .
$$

Logo $T(t-s) v(s)$ não depende de $s$. Tomamos assim, $s=0$ e $s=t$ a fim de obtermos

$$
T(t-0) v(0)=T(t-t) v(t)
$$

ou seja, $v(t)=T(0) v(t)=T(t) u_{0}$. 
Reciprocamente, supomos que 3.29 está bem posto e tomamos $\lambda \in \rho(A)$. Podemos supor, sem perda de generalidade, $\lambda=0$. De fato, se $\lambda \neq 0$, substituimos $A$ por $B=A-\lambda I$ e assim, uma função $u$ é solução de 3.29 se, e somente se, $v(t)=e^{-\lambda t} u(t)$ é solução de

$$
\begin{array}{r}
v_{t}=B v(t), \quad \forall t>0 \\
v(0)=u_{0} .
\end{array}
$$

além disso, $0 \in \rho(B)$. Sendo então $\lambda=0$ prosseguimos definindo os seguintes espaços de Banach:

$$
\mathcal{X}=C^{1}([0,1] ; X) \text { com a norma }\|u\|_{\mathcal{X}}=\sup _{t \in[0,1]}\left(\|u(t)\|+\left\|u_{t}(t)\right\|\right)
$$

e

$$
\mathcal{Y}=D(A) \text { com a norma }\|v\|_{\mathcal{Y}}=\|A v\| .
$$

Consideremos a aplicação $S: \mathcal{Y} \longrightarrow \mathcal{X}$ dada por $S(v)=u$, onde $u$ é a única solução de

$$
\begin{array}{r}
u_{t}=A u(t), \quad \forall t>0 \\
u(0)=v .
\end{array}
$$

Pela linearidade do problema de Cauchy acima e unicidade da solução, concluímos que $S$ é um operador linear definido em todo o espaço $D(A)$. Afirmamos que $S$ é um operador fechado. De fato, considerando uma seqüência $\left(v_{n}\right)$ em $\mathcal{Y}$ tal que $v_{n} \longrightarrow 0$ e $S\left(v_{n}\right) \longrightarrow u$, é suficiente provar que $u=S(0)=0$. Sendo $A$ um operador fechado, então

$$
S\left(v_{n}\right)(t) \doteq u_{n}(t) \longrightarrow u(t) \text { e } A u_{n}(t)=\left(u_{n}\right)_{t}(t) \longrightarrow u_{t}(t) \Rightarrow u(t) \in D(A) \text { e } u_{t}(t)=A u(t) .
$$

Obtemos ainda que $u(0)=\lim _{n \rightarrow \infty} u_{n}(0)=0$ e, portanto, $u \equiv 0$. O Teorema do Gráfico Fechado nos permite então concluir que $S$ é um operador limitado. Definimos a aplicação $T(t): \mathcal{Y} \longrightarrow \mathcal{Y}$ por $T(t) v=S(v)(t)$. Sendo $S$ limitado, então

$$
\sup _{t \in[0,1]}\left(\|u(t)\|+\left\|u_{t}(t)\right\|\right) \leq\|S\|\left\|A u_{0}\right\|
$$

Logo, a família $\{T(t): t \geq 0\}$ é um semigrupo fortemente contínuo em $D(A)$, uma vez que $\|T(t)\| \leq$ $\|L\|$ para $t \in[0,1]$.

Estudaremos agora o caso não homogêneo. Considere então o seguinte problema

$$
\begin{array}{r}
u_{t}=A u(t)+f(t), \quad \forall t>0 \\
u(0)=u_{0},
\end{array}
$$

onde $A$ é um operador linear fechado e densamente definido em $X, u_{0} \in X$ e $f:[0, T) \longrightarrow X$, para $\operatorname{algum} T \in \mathbb{R}^{+}$. 
Para o problema de Cauchy abstrato não homogêneo 3.30 introduziremos também duas noções de solução e apresentaremos algumas relações entre elas.

Definição 33 Dizemos que uma função $u:[0, T) \longrightarrow X$ é uma solução clássica de 3.30 em $[0, T)$ se u é contínua em $[0, T)$, continuamente diferenciável em $(0, T), u(t) \in D(A)$ para $t \in(0, T)$ e $u$ satisfaz $3.30 \mathrm{em}[0, T)$.

Definição 34 Seja $A$ é gerador de um semigrupo fortemente contínuo $T(t)$ e $\int_{0}^{T}\|f(t)\| d t<\infty$. Dizemos que $u \in C([0, T] ; X)$ é uma solução fraca de $3.30 \mathrm{em}[0, T]$, se

$$
u(t)=T(t) u_{0}+\int_{0}^{t} T(t-s) f(s) d s, \quad \forall t \in[0, T]
$$

para cada $x \in X$.

Observamos que se $f \equiv 0$ a definição de solução fraca para 3.30 coincide com aquela correspondente ao caso homogêneo.

Proposição 6 Sejam A gerador de um semigrupo fortemente contínuo e $\int_{0}^{T}\|f(t)\| d t<\infty$ então, para cada $u_{0} \in X$, o problema de Cauchy abstrato 3.30 tem no máximo uma solução (clássica). Além disso, se existir uma solução (clássica) então ela é uma solução fraca.

Demonstração. Suponha a princípio que exista uma solução (clássica) de 3.30, mas que não seja única. Denotaremos por $u$ e $v$ duas soluções e por $w$ a diferença entre elas, ou seja, $w=u-v$. Então,

$$
\begin{array}{r}
w_{t}=A w(t), \quad \forall t>0 \\
w(0)=0,
\end{array}
$$

Uma vez que $w \equiv 0$ é uma solução do problema acima e, pelo teorema 24 , se existir uma solução então ela é única, concluímos que $u \equiv v$.

Suponha que exista uma solução clássica $u$ de 3.30, mostraremos que ela é dada por 3.31. Seja $T(t)$ o semigrupo fortemente contínuo gerado por $A$, então a função $g:[0, \infty) \longrightarrow X$ dada por $g(s)=T(t-s) u(s)$ é diferenciável e, para $0<s<t$,

$$
\begin{aligned}
\frac{d g}{d s} & =T(t-s) u_{t}(s)-A T(t-s) u(s) \\
& =T(t-s) A u(s)+T(t-s) f(s)-A T(t-s) u(s) \\
& =T(t-s) f(s) .
\end{aligned}
$$

Sendo $\int_{0}^{T}\|f(t)\| d t<\infty$, então $T(t-s) f(s)$ é integrável e, portanto,

$$
\int_{0}^{t} \frac{d g}{d s} d s=\int_{0}^{t} T(t-s) f(s) d s
$$


ou seja,

$$
u(t)=T(t) u_{0}+\int_{0}^{t} T(t-s) f(s) d s
$$

Sabemos que se $A$ for gerador de um semigrupo fortemente contínuo $T(t)$ e $\int_{0}^{T}\|f(t)\| d t<\infty$ então o problema 3.30 tem uma solução fraca. Estamos interessados agora nos casos em que, além de existir, tal solução é também clássica. Mais precisamente, queremos saber em que condições o problema 3.30 está bem posto. Este é o conteúdo do próximo teorema.

Teorema 25 Sejam $A$ um gerador infinitesimal de um semigrupo analítico em $X$ e $u_{0} \in D(A)$. Então 3.30 tem uma única solução (clássica) $u \in C^{1}([0, \infty) ; X)$, com $u(t) \in D(A)$ para todo $t \in(0, \infty)$, se $f$ satisfaz pelo menos uma das seguintes afirmações:

1. $f \in C([0, \infty) ; X), f(t) \in D(A)$ para todo $t \in(0, T)$ e Af $\in C([0, T) ; X)$;

2. $f \in C^{1}((0, T) ; X)$.

Demonstração. Sabemos da proposição 6 que, se existir uma solução (clássica) de 3.30, então ela deve ser única e da forma 3.31. Uma vez que $A$ é gerador de um semigrupo fortemente contínuo e, assumindo 1 ou 2, pode-se ainda obter que $\int_{0}^{T}\|f(t)\| d t<\infty$ para $T \in(0, \infty)$, concluímos que

$$
u(t)=T(t) u_{0}+\int_{0}^{t} T(t-s) f(s) d s, \quad \forall t \in[0, T]
$$

é de fato uma solução (fraca). Nosso próximo passo é garantir que $u$ como na definição 34 pertence a $C^{1}([0, \infty) ; X)$ e que $u(t) \in D(A)$, se $t \in(0, \infty)$.

Prosseguimos definindo a seguinte função,

$$
v(t) \doteq \int_{0}^{t} T(t-s) f(s) d s
$$

É claro que, $u \in C^{1}([0, \infty) ; X)$ se, e somente se, $v \in C^{1}([0, \infty) ; X)$. Além disso, em ambos os casos obteríamos

$$
u_{t}(t)=A T(t) u_{0}+v_{t}(t) .
$$

Portanto, se $u(0)=u_{0}$,

$$
u_{t}(t)=A u(t)+f(t) \Longleftrightarrow v_{t}(t)=A u(t)+f(t)-A T(t) u_{0}=A v(t)+f(t),
$$

já que $A u(t)+f(t)=u_{t}(t)=A T(t) u_{0}+v_{t}(t)$.

Suponhamos inicialmente que a afirmação 1 seja válida. Então $v(t) \in D(A)$ e assim obtemos que $u(t) \in D(A)$, se $t>0$. Além disso, $v \in C^{1}([0, \infty))$ e

$$
v_{t}(t)=T(0) f(t)+\int_{0}^{t} \frac{\partial}{\partial t} T(t-s) f(s) d s
$$




$$
\begin{aligned}
& =f(t)+\int_{0}^{t} T(t-s) A f(s) d s \\
& =f(t)+A v(t)
\end{aligned}
$$

pois $A v(t)=\int_{0}^{t} T(t-s) A f(s) d s$.

Se a afirmação 2 for válida, então observamos a princípio que

$$
\int_{a}^{b} T(t) y d t \in D(A) e A \int_{a}^{b} T(t) y d t=T(b) y-T(a) y
$$

para todo $y \in X$ e $a, b \in \mathbb{R}$. Uma vez que o lema anterior não pode ser aplicado diretamente, escreveremos $v$ da seguinte forma:

$$
\begin{aligned}
v(t) & =\int_{0}^{t} T(t-s)\left(f(0)+\int_{0}^{s} f_{t}(r) d r\right) d s \\
& =\int_{0}^{t} T(t-s) f(0) d s+\int_{0}^{t} \int_{r}^{t} T(t-s) f_{t}(r) d s d r \\
& =\int_{0}^{t} T(\tau) f(0) d \tau+\int_{0}^{t} \int_{0}^{t-r} T(\tau) f_{t}(r) d \tau d r
\end{aligned}
$$

e então, podemos concluir que $v(t) \in D(A)$ para todo $t>0$. Vale ainda que $v \in C^{1}([0, \infty) ; X)$ e

$$
\begin{aligned}
v_{t}(t) & =\frac{d}{d t} \int_{0}^{t} T(\tau) f(t-\tau) d \tau \\
& =T(t) f(0)+\int_{0}^{t} T(\tau) f_{t}(t-\tau) d \tau \\
& =T(t) f(0)+\int_{0}^{t} T(t-s) f_{t}(s) d s .
\end{aligned}
$$

Por outro lado,

$$
\begin{aligned}
A v(t) & =T(t) f(0)-f(0)+\int_{0}^{t}\left[-f_{t}(s)+T(t-s) f_{t}(s)\right] d s= \\
& =T(t) f(0)-f(0)-f(t)+f(0)+\int_{0}^{t} T(t-s) f_{t}(s) d s .
\end{aligned}
$$

Combinando as identidades acima, obtem-se o resultado desejado.

Descrevemos a seguir condições para que o problema de valor inicial 3.30 tenha uma única solução (clássica) para todo $x \in X$. Uma das condições envolve o conceito de função Hölder contínua e, portanto, apresentamos inicialmente a seguinte

Definição 35 Sejam $X$ um espaço de Banach, $I=[a, b] \subset \mathbb{R}$ e $f: I \longrightarrow X$. Dizemos que $f$ é 
uma função Hölder contínua se existirem constantes reais $K$ e $\eta$, com $0<\eta \leq 1$, tais que

$$
\|f(t)-f(s)\| \leq K|t-s|^{\eta}, \quad \forall s, t \in I .
$$

Exemplo 6 Toda função Lipschtziana é Hölder contínua. Basta tomarmos $\eta=1$ e $K=C_{\text {Lip }}$.

Exemplo 7 Seja $f_{\alpha}: I \longrightarrow \mathbb{R}$ dada por

$$
f(x)=\alpha x .
$$

Então,

$$
|\alpha x-\alpha y| \leq|\alpha||x-y|
$$

e, assim, se tomarmos $K=|\alpha|$ e $\eta=1$, segue que $\left\{f_{\alpha} ; \alpha \in \mathbb{R}\right\}$ é uma família de funções Hölder contínuas.

Teorema 26 Sejam A um operador setorial, $f$ uma função Hölder contínua em [0,T], para cada $T \in(0, \infty)$, e $u_{0} \in X$. Então 3.30 tem uma única solução (clássica) $u \in C([0, \infty) ; X) \cap$ $C^{1}((0, \infty) ; X)$. Além disso, se $u_{0} \in D(A)$ então $u \in C^{1}([0, \infty) ; X)$.

Demonstração. Seja $T(t)$ o semigrupo gerado por $A$. Então $T(t)(X) \in D(A)$ para $t>0$ e ainda, pela demonstração do teorema 25 , é suficiente provar que $v:[0, \infty) \longrightarrow D(A)$ e $A v \in C([0, \infty) ; X)$ onde

$$
v(t)=\int_{0}^{t} T(t-s) f(s) d s
$$

para $t \in[0, \infty)$. Sejam

$$
v_{1}(t)=\int_{0}^{t} T(t-s) f(t) d s=\int_{0}^{t} T(r) f(t) d r
$$

$\mathrm{e}$

$$
v_{2}(t)=\int_{0}^{t} T(t-s)[f(s)-f(t)] d s,
$$

então $v=v_{1}+v_{2}$. Observamos que $v_{1}(t) \in D(A)$ e, uma vez que $A v_{1}(t)=[T(t)-I] f(t)$, $A v_{1}$ é contínua em $[0, \infty)$. A fim de obtermos as mesmas conclusões para $v_{2}$, faremos a seguinte aproximação

$$
\lim _{\epsilon \rightarrow 0} w_{\epsilon}(t)=v_{2}(t)
$$

onde

$$
w_{\epsilon} \doteq\left\{\begin{array}{cc}
\int_{0}^{t-\epsilon} T(t-s)[f(s)-f(t)] d s & \text { se } t \geq \epsilon \\
0 & \text { se } 0 \leq t \leq \epsilon .
\end{array}\right.
$$

De fato $w_{\epsilon}$ converge uniformemente em $t$ para $v_{2}(t)$ em qualquer subconjunto limitado de $[0, \infty)$.

Obtemos de

$$
A T(t-s)[f(s)-f(t)]=[A T(\epsilon)] T(t-s-\epsilon)[f(s)-f(t)]
$$


que $w_{\epsilon}(t) \in D(A)$ e, portanto,

$$
A w_{\epsilon}(t)=\int_{0}^{t-\epsilon} A T(t-s)[f(s)-f(t)] d s
$$

Sendo $f$ Hölder contínua e $\|A T(t-s)\| \leq \frac{C}{(t-s)}$, para $0 \leq<t<T$ e para alguma constante $C=C(T)$, obtemos

$$
\lim _{\epsilon \rightarrow 0} A w_{\epsilon}=\int_{0}^{t} A T(t-s)[f(s)-f(t)] d s .
$$

Como $A$ é fechado, $v_{2}(t) \in D(A)$ e

$$
A v_{2}(t)=\int_{0}^{t} A T(t-s)[f(s)-f(t)] d s
$$

Resta-nos provar que $A v_{2} \in C([0, \infty) ; X)$. É claro que, $A v_{2}$ é contínua em $t=0$. Vamos supor então que $t>0$ e, assim

$$
A v_{2}(t)=J_{1}(t)+J_{2}(t)
$$

onde $J_{1}(t)=\int_{0}^{\delta} A T(t-s)[f(s)-f(t)] d s, J_{2}(t)=\int_{\delta}^{t} A T(t-s)[f(s)-f(t)] d s$ e $\delta \in(0, t)$. Para cada $\delta$ fixo, $J_{2} \in C([0, \infty) ; X)$ e $\left\|J_{1}(t)\right\|=O(\delta)$, em cada intervalo da forma $[0, T]$. Logo, $A v_{2}$ é contínua em $[0, \infty)$.

\subsection{O problema de Cauchy semilinear}

O principal objetivo desta seção é apresentar alguns resultados acerca da existência, unicidade e regularidade de soluções do problema de Cauchy semilinear, com o intuito de, no próximo capítulo, discutirmos estes mesmos aspectos mas do particular caso 2.30- 2.32.

Considere então um espaço de Banach $X$ e um operador setorial $A: D(A) \subset X \longrightarrow X$. Pode-se então definir as potências fracionárias de $A_{1} \doteq A+a I$ e ainda os espaços $X^{\alpha}=D\left(A_{1}^{\alpha}\right)$ com a norma do gráfico $\|x\|_{\alpha}=\left\|A_{1}^{\alpha}\right\|$, para $\alpha>0$ e $a \geq 0$. Seja ainda uma função $f: U \subset\left(\mathbb{R} \times X^{\alpha}\right) \longrightarrow X$, para algum $\alpha \in[0,1)$, contínua. Queremos estudar a boa colocação do seguinte problema de valor inicial

$$
\begin{array}{r}
u_{t}=-A u(t)+f(t, u), \quad \forall 0<t<t_{1} \\
u(0)=u_{0},
\end{array}
$$

onde $u_{0} \in X^{\alpha}$. O sinal negativo indica apenas que queremos resolver o problema de Cauchy abstrato para o caso parabólico, uma vez que o sinal contorna alguns problemas de notação relacionados ao uso das potências fracionárias de $A .{ }^{14}$

Como no caso linear, vamos inicialmente discutir os conceitos de soluções clássica e fraca, desta vez para o problema 3.32. Inicialmente apresentamos a seguinte

\footnotetext{
${ }^{14} \mathrm{O}$ mesmo não ocorre no caso semilinear hiperbólico, uma vez que este tipo de problema não tem como condição que o operador $A$ seja setorial e, portanto, não faz sentido falar em potências fracionárias, já que estas não estariam definidas.
} 
Definição 36 Sejam $U$ um subconjunto aberto de $\mathbb{R}^{+} \times X^{\alpha}$ e $f: U \longrightarrow X$. Dizemos que $f$ é Hölder contínua em $t$ e Lipschitziana em u se existirem contantes $K$ e $\eta$, com $0<\eta \leq 1$, tais que

$$
\left\|f\left(t_{1}, u_{1}\right)-f\left(t_{2}, u_{2}\right)\right\| \leq K\left(\left|t_{1}-t_{2}\right|^{\eta}+\left\|u_{1}-u_{2}\right\|_{\alpha}\right),
$$

para quaisquer $\left(t_{1}, u_{1}\right)$ e $\left(t_{2}, u_{2}\right)$ em $U$.

Definição 37 Sejam $A$ um operador setorial e $f: U \subset \mathbb{R} \times X^{\alpha} \longrightarrow X$. Dizemos que uma função contínua $u:\left[t_{0}, t_{1}\right) \longrightarrow X$ é uma solução fraca de 3.32 se u for solução da seguinte equação integral

$$
u(t)=e^{-A\left(t-t_{0}\right)} u_{0}+\int_{t_{0}}^{t} e^{-A(t-s)} f(s, u(s)) d s
$$

A equação 3.33 é conhecida como Fórmula da Variação das Constantes.

Definição 38 Se A é um operador setorial e $f: U \subset \mathbb{R} \times X^{\alpha} \longrightarrow X$ localmente Hölder contínua em $t$, então uma solução (clássica) do problema de valor inicial 3.32 é uma função contínua $u$ : $\left[t_{0}, t_{1}\right) \longrightarrow X$ tal que $u\left(t_{0}\right)=u_{0}$ e em $\left(t_{0}, t_{1}\right)$ vale que $(t, u(t)) \in U, u(t) \in D(A)$, u é diferenciável, $\int_{t_{0}}^{t}(t-s)^{-\alpha}\|f(t, u(t))\| d t \rightarrow \infty$, quando $t \rightarrow t_{0}^{+}$, e $u$ ainda satisfaz a equação diferencial em 3.32.

O próximo resultado mostra que no caso semilinear o conceito de solução fraca não se faz necessário, uma vez que verificaremos que as definições acima são equivalentes ${ }^{15}$.

Lema 13 Suponha que $A: D(A) \subset X \longrightarrow X$ seja um operador setorial e $f$ uma função localmente Hölder contínua em $t$ e localmente Lipschitziana em u. Então as seguintes afirmações são válidas:

1. se u é uma solução clássica de $3.32 \mathrm{em}\left(t_{0}, t_{1}\right)$ então u é uma solução fraca.

2. se $u:\left[t_{0}, t_{1}\right) \longrightarrow X^{\alpha}$ é uma função contínua tal que $(t, u(t)) \in U$, para todo $t \in\left[t_{0}, t_{1}\right]$, $\int_{t_{0}}^{t}(t-s)^{-\alpha}\|f(t, u(t))\| d t \rightarrow \infty$ quando $t \rightarrow t_{0}^{+} e$

$$
u(t)=e^{-A\left(t-t_{0}\right)} u_{0}+\int_{t_{0}}^{t} e^{-A(t-s)} f(s, u(s)) d s,
$$

então u é uma solução clássica de 3.32.

\section{Demonstração.}

1. De maneira análoga ao que foi feito em argumentos anteriores, denotaremos por $T(t)$ o semigrupo gerado por $A$ e por $g$ a função dada por $g(s)=T(t-s) u(s)$, onde $u$ é solução clássica de 3.32. Também neste caso concluiremos que $g$ é diferenciável, para $0<s<t$, tem-se $\frac{d g}{d s}=T(t-s) f(s, u(s))$. Uma vez que, pela definição de solução clássica, deve valer que

\footnotetext{
${ }^{15}$ Os conceitos de solução fraca e clássica somente coincidem para o caso semilinear parabólico. É possível verificar que o mesmo não ocorre para o caso semilinear hiperbólico [35] e [7].
} 
$\int_{t_{0}}^{t}(t-s)^{-\alpha}\|f(t, u(t))\| d t \rightarrow \infty$ quando $t \rightarrow t_{0}^{+}$, pode-se concluir que $T(t-s) f(s, u(s))$ é integrável. Segue então que

$$
\int_{t_{0}}^{t} \frac{d g}{d s}=\int_{t_{0}}^{t} T(t-s) f(s, u(s))
$$

ou seja, $u$ é uma solução fraca de 3.32 .

2. Sendo $u$ uma solução da equação integral 3.33, então $u \in C\left(\left(t_{0}, t_{1}\right) ; X^{\alpha}\right)$. Nos resta apenas provar que $g(t)=f(t, u(t))$ é localmente Hölder contínua e que $(t, u(t)) \in U$, para todo $t \in\left(t_{0}, t_{1}\right)$. Veremos inicialmente que $u$ é localmente Hölder contínua. Seja $B=$ $\sup _{\left[t_{0}, t_{1}\right]}\|f(t, u(t))\| \leq B$. É claro que $B<\infty$, já que $\left[t_{0}, t_{1}\right]$ é compacto e $u$ é contínua. Então, para $t_{0}<t \leq t+h \leq t_{1}$, vale que

$$
\begin{aligned}
u(t+ & h)-u(t)=\left(e^{-A h}\right)-I\left[e^{-A\left(t-t_{0}\right)} u\left(t_{0}\right)+\int_{t_{0}}^{t} e^{-A(t-s)} f(s, e(s)) d s\right] \\
& +\int_{t}^{t+h} e^{-A(t+h-s)} f(s, u(s)) d s .
\end{aligned}
$$

De onde obtemos que, se $0<\delta<1-\alpha$,

$$
\begin{aligned}
\| u(t & +h)-u(t)\left\|_{\alpha} \leq M h^{\delta}\left(t-t_{0}\right)^{-\delta}\right\| u\left(t_{0}\right) \|_{\alpha}+\int_{t_{0}}^{t} M h^{\delta}(t-s)^{-\alpha-\delta} B d s \\
& +\int_{t}^{t+h} M(t+h-s)^{-\alpha} B d s \\
& =O\left(h^{\delta}\left(t-t_{0}\right)\right)^{-\delta},
\end{aligned}
$$

onde $M$ é a constante dada pelo teorema 5. Como $u$ é dada por ??e concluímos ainda que $u$ é localmente Hölder contínua, então $g(t)$ é contínua e ainda satisfaz

$$
\|g(t+h)-g(t)\| \leq K\left(t-t_{0}\right)^{-\eta} h^{\eta}
$$

para constantes $K$ e $\eta$. Logo, $u$ satisfaz 3.32 e, portanto, é uma solução clássica.

Vimos então que, se existir uma solução (clássica) de 3.32 então ela é dada por 3.33. O que nos interessa agora é saber em que condições o problema de valor inicial semilinear de fato tem uma solução. Tais condições são apresentadas no teorema abaixo.

Teorema 27 Suponha que $A$ seja um operador setorial e, para algum $\alpha \in[0,1), f: U \longrightarrow X$, onde $U$ é um aberto em $\mathbb{R} \times X^{\alpha}$ seja uma função localmente Hölder contínua em $t$ e localmente 
Lipschitziana em u. Então, dado qualquer $\left(t_{0}, u_{0}\right) \in U$, existe $\tau=\tau\left(t_{0}\right)$, tal que 3.32 tem uma única solução u em $\left(t_{0}, \tau\right)$.

Demonstração. Pelo lema anterior, é suficiente provarmos o resultado para a equação diferencial 3.33. Mais precisamente, basta verificar a existência de uma solução da equação dada pela fórmula da variação das constantes, uma vez que sabemos que se solução existe então ela é única. Fixando então $\left(t_{0}, u_{0}\right) \in U$ e escolhendo constantes $\delta>0$ e $\bar{t}>t_{0}$ de modo que o conjunto

$$
V=\left\{(t, x): t \in\left[t_{0}, \bar{t}\right],\left\|u-u_{0}\right\|_{\alpha} \leq \delta\right\} \subset U
$$

satisfaça

$$
\left\|f\left(t, u_{1}\right)-f\left(t, u_{2}\right)\right\| \leq\left\|u_{1}-u_{2}\right\|_{\alpha},
$$

para $\left(t, u_{1}\right),\left(t, u_{2}\right) \in V$. Sejam ainda $B=\max _{\left[t_{0}, \bar{t}\right]}$ e $\tau \in(0, \bar{t}]$, de modo que

$$
\left\|\left(e^{-A h}-I\right) u_{0}\right\|_{\alpha} \leq \frac{\delta}{2}, \forall h \in[0, \tau]
$$

e

$$
M(B+L \delta) \int_{0}^{\tau} z^{-\alpha} e^{a z} d z \leq \frac{\delta}{2}
$$

onde $M$ é tal que $\|\left(A_{1}^{\alpha} e^{-A t} \| \leq M t^{-\alpha} e^{a t}\right.$ para $t>0$.

Considere o espaço de Banach $Y \doteq C\left(\left[t_{0}, \tau\right] ; X^{\alpha}\right)$ com a norma

$$
\|y\|_{Y}=\sup _{\left[t_{0}, \tau\right]}\|y(t)\|_{\alpha}
$$

Para cada $y \in Y$, definimos $G(y):\left[t_{0}, \tau\right] \longrightarrow X$ por

$$
G(y)(t)=e^{-A\left(t-t_{0}\right)} u_{0}+\int_{t_{0}}^{t} e^{-A(t-s)} f(s, y(s)) d s .
$$

Considere agora o seguinte subconjunto fechado, não vazio e limitado de $Y$,

$$
S \doteq\left\{y \in Y:\left\|y(t)-u_{0}\right\|_{\alpha} \leq \delta\right\}
$$

Uma vez que $S$ é um subconjunto fechado do espaço de Banach $Y, S$ é também um espaço de Banach. Afirmamos que $G(S) \subset S$. De fato,

$$
\begin{aligned}
& \left\|G(y)(t)-u_{0}\right\|_{\alpha} \leq\left\|\left(e^{-A\left(t-t_{0}\right)}-I\right) u_{0}\right\|_{\alpha}+\int_{t_{0}}^{t}\left\|A_{1}^{\alpha} e^{-A(t-s)}\right\|(B+L \delta) d s \\
& \quad \leq \frac{\delta}{2}+M(B+L \delta) \int_{t_{0}}^{\tau}(t-s)^{-\alpha} e^{a(t-s) d s} \\
& \quad \leq \delta
\end{aligned}
$$

e $G(y) \in C\left(\left[t_{0}, \tau\right] ; X^{\alpha}\right)$. 
Além disso, se $y_{1}, y_{2} \in S$ então

$$
\begin{aligned}
& \left\|G\left(y_{1}\right)(t)-G\left(y_{2}\right)(t)\right\|_{\alpha} \leq \int_{t_{0}}^{t}\left\|A_{1}^{\alpha} e^{-A(t-s)}\right\|\left\|f\left(s, y_{1}(s)\right)-f\left(s, y_{2}(s)\right)\right\| d s \\
& \leq M L \int_{t_{0}}^{t}(t-s)^{-\alpha} e^{a(t-s)} d s\left\|y_{1}-y_{2}\right\|_{Y},
\end{aligned}
$$

de onde obtemos

$$
\left\|G\left(y_{1}\right)-G\left(y_{2}\right)\right\|_{Y} \leq \frac{1}{2}\left\|y_{1}-y_{2}\right\|_{Y}, \forall y_{1}, y_{2} \in S .
$$

Provamos então que $G$ é uma contração no espaço de Banach $S$ e, portanto, pelo Teorema da Contração ${ }^{16}, G$ tem um único ponto fixo $u$ em $S$. Então $u$ satisfaz a equação integral ?? e, que está bem definida. De fato, sendo $u$ contínua e $f$ Hölder contínua, $t \longmapsto f(t, u(t))$ é contínua em $\left[t_{0}, \tau\right]$ e, portanto, limitada nesse intervalo.

Vimos acima que, impondo algumas condições acerca da função $f$, é possível obter (localmente) uma solução única continuamente diferenciável do problema de valor inicial (3.32). O que o corolário a seguir nos mostra é que, além disso, a derivada $u_{t}$ da solução é localmente Hölder contínua em $\left(t_{0}, t_{1}\right)$.

Corolário 3 Se o operador A e a função $f$ satisfazem as mesmas condições impostas pelo teorema anterior e, além disso, $f$ for Hölder contínua em $U^{17}$ com expoentes $\eta$, então $u_{t}$ é localmente Hölder contínua em $\left[t_{0}, t_{1}\right)$ com expoente $\vartheta=\min (\eta, \beta)$, para qualquer $\beta \in(0,1-\alpha)$, onde u é a solução de $3.32 \mathrm{em}\left[t_{0}, t_{1}\right]$.

A demonstração desta afirmação pode ser encontrada à página 199 de [35].

Obtemos a seguir um resultado acerca da extensão da soluções de 3.32 e ainda da existência de intervalos maximais. Tal resultado, como veremos, será essencial no estudo do comportamento assintótico de soluções de 3.32 uma vez que nos permitirá obter a existência global de soluções. Antes apresentaremos um fato que nos será útil na demonstração deste último resultado.

Considere duas constantes não-negativas $a$ e $b$ e suponha que $u(t)$ seja tal que

$$
0 \leq u(t) \leq a+b \int_{0}^{t} u(s) d s
$$

para todo $0 \leq t \leq T$. Então,

$$
u(t) \leq a e^{b t}
$$

para todo $t \in[0, T)$. Este resultado é uma formulação elementar da conhecida desigualdade de Gronwall ${ }^{18}$.

\footnotetext{
${ }^{16} \mathrm{O}$ Teorema da Contração é também conhecido na literatura como Teorema do Ponto Fixo de Banach. Uma referência bastante útil a este respeito é [26].

${ }^{17}$ Observe que nos resultados e definições até aqui a função $f$ era apenas localmente Hölder contínua.

${ }^{18} \operatorname{Ver}[26]$.
} 
Lema 14 Seja $g:[0, T] \times[0, T] \longrightarrow \mathbb{R}$, para algum $T \geq 0$, tal que $g(t, s) \geq 0$ e g é contínua se $s<t$. Se existirem contantes positivas $a, b$ e $\alpha$ tais que

$$
g(t, s) \leq a+b \int_{t}^{s}(t-\tau)^{\alpha-1} g(\tau, s) d \tau, \quad \forall s<t,
$$

então existe uma constante $C$ tal que $g(t, s) \leq C$ para todo $(t, s) \in[0, T] \times[0, T]$ com $s<t$.

Demonstração. Usaremos na demonstração a seguinte identidade

$$
\int_{t}^{s}(t-\tau)^{\alpha-1}(\tau-s)^{\beta-1} d \tau=(t-s)^{\alpha+\beta-1} \frac{\Gamma(\alpha) \Gamma(\beta)}{\Gamma(\alpha+\beta)}
$$

onde $\alpha, \beta>0$ e $\Gamma(\cdot)$ é a função dada pela definição 26 .

Iterando $3.34(n-1)$ vezes usando a identidade 3.35 e o fato de que $t-s<T$ obtemos

$$
g(t, s) \leq a \sum_{j=0}^{n-1}\left(\frac{b T^{\alpha}}{\alpha}\right)^{j}+\frac{(b \Gamma(\alpha))^{n}}{\Gamma(n \alpha)} \int_{s}^{t}(t-\tau)^{n \alpha-1} g(\tau, s) d \tau
$$

Escolhemos então $n$ suficientemente grande, de modo que $n \alpha>1$ e $(t-\tau)^{n \alpha-1}<T^{n \alpha-1}$. Assim obtemos

$$
g(t, s) \leq c_{1}+c_{2} \int_{s}^{t} g(\tau, s) d \tau,
$$

onde $c_{1}$ e $c_{2}$ são contantes positivas. Portanto, pela desigualdade de Gronwall, obtemos

$$
g(t, s) \leq c_{1} e^{c_{2}(t-s)} \leq c_{1} e^{c_{2} T} \leq C,
$$

para alguma constante $C$. Uma vez que as contantes $c_{1}$ e $c_{2}$ não dependem de $s$, concluímos o resultado.

Teorema 28 Suponha que $A$ e $f$ satisfazem as hipóteses do teorema 27. Assuma também que, para todo subconjunto fechado $B$ de $U$, a imagem $f(B)$ é limitada em $X$. Seja u uma solução de $3.32 \mathrm{em}\left(t_{0}, t_{1}\right)$ e suponha ainda que $t_{1}$ é maximal, ou seja, que não existe uma solução de 3.32 em $\left(t_{0}, \bar{t}\right)$ se $\bar{t}>t_{1}$. Se $t_{1}<\infty$ então $(t, u(t)) \longrightarrow \partial U$ se $t \longrightarrow t_{1}$.

Demonstração. Suponhamos que $(t, u(t))$ não converge para $\partial U$. Então existe uma vizinhança $N$ de $\partial U$ tal que $(t, u(t)) \notin N$ para todo $t \in\left[t_{2}, t_{1}\right)$, para algum $t_{2}<t_{1}$. Podemos supor que $N$ é forma $U \backslash B$ para algum subconjunto fechado e limitado $B$ de $U$. E assim, $(t, u(t)) \in B$ para $t \in\left[t_{2}, t_{1}\right)$.

Se provarmos que existe $u_{1} \in B$ tal que $u(t) \longrightarrow u_{1}$ em $X^{\alpha}$, se $t \longrightarrow t_{1}^{-}$, então $t_{1}$ não seria maximal, uma vez que poderíamos aplicar o teorema 27 e concluir que existe uma solução da equação em $3.32 \operatorname{com} u\left(t_{1}\right)=u_{1}$. 
Observe ainda que, se $t_{2} \leq \bar{t}<t<t_{1}$, então

$$
u(t)-u(\bar{t})=\left[e^{-A(t-\bar{t})}-I\right] u(\bar{t})+\int_{\bar{t}}^{t} e^{-A(t-s) f(s, u(s)) d s},
$$

e portanto,

$$
\begin{aligned}
& \|u(t) u(\bar{t})\|_{\alpha} \leq C_{1}(t-\bar{t})^{\beta-\alpha}\|u(\bar{t})\|_{\beta}+C_{2} \int_{\bar{t}}^{t}(t-s)^{-\alpha} d s \\
& \quad \leq C_{3}(t-\bar{t})^{\beta-\alpha},
\end{aligned}
$$

onde $\beta \in(\alpha, 1)$ e $C_{1}, C_{2}, C_{3}$ são constantes. Se provarmos que $\|u(t)\|_{\beta}$ é limitado concluiremos que existe o limite $\lim _{t \rightarrow t_{1}} u(t)$ em $X^{\alpha}$.

Sejam $\beta \in(\alpha, 1)$ e $t \in\left[t_{2}, t_{1}\right]$, então, uma vez que $f(B)$ é limitado, obtemos

$$
\begin{gathered}
\|u(t)\|_{\beta} \leq\left\|A_{1}^{\beta-\alpha} e^{-A\left(t-t_{0}\right)}\right\|\left\|u\left(t_{0}\right)\right\|_{\alpha}+\int_{t_{0}}^{t}\left\|A_{1}^{\beta} e^{-A(t-s)}\right\|\|f(s, u(s))\| d s \\
\leq C\left[\left(t-t_{0}\right)^{-(\beta-\alpha)}\left\|u\left(t_{0}\right)\right\|_{\alpha}+\int_{t_{0}}^{t}(t-s)^{-\beta} d s\right],
\end{gathered}
$$

onde $C$ é uma constante. Logo a demonstração está completa.

Corolário 4 Sejam $A$ um operador setorial e $f: U=\left[t_{0}, \infty\right) \times X^{\alpha} \longrightarrow X$ localmente Hölder contínua em $t$ e localmente Lipschitziana em $x$ para $(t, x) \in U$. Se existe uma função contínua não-decrescente $K:\left[t_{0}, \infty\right) \longrightarrow \mathbb{R}$ tal que

$$
\|f(t, u)\| \leq K(t)\left(1+\|u\|_{\alpha}\right), \text { para todo }(t, u) \in U,
$$

então, para todo $u_{0} \in X^{\alpha}$, a única solução de 3.32 existe para todo $t \geq t_{0}$.

Demonstração. De acordo com o teorema anterior, é suficiente provar que existe o limite $u_{1}=$ $\lim _{t \rightarrow t_{1}^{-}} u(t)$, de modo que $\left(t_{1}, u_{1}\right) \in B$ e então concluiremos que $t_{1}=\infty$. De maneira análoga ao que foi feito na demonstração anterior, basta ver que $\|u(t)\|_{\alpha}$ permanece limitada se $t \rightarrow t_{1}$.

Uma vez que

$$
A^{\alpha} u(t)=A^{\alpha} T\left(t-t_{0}\right) u_{0}+\int_{t_{0}}^{t} A^{\alpha} T(t-s) f(s, u(s)) d s
$$

e $K(t)$ é não decrescente, é possível obter

$$
\|u(t)\|_{\alpha} \leq M\left\|A^{\alpha} u_{0}\right\|+\frac{K\left(t_{1}\right) t_{1}^{1-\alpha}}{1-\alpha}+K\left(t_{1}\right) \int_{t_{0}}^{t}(t-s)^{-\alpha}\|u(s)\|_{\alpha} d s
$$

onde $T(t)$ é o semigrupo gerado por $A$ e $M$ é a constante dada pelo teorema 5. Portanto, aplicando o lema 14 concluímos que $\|u(t)\|_{\alpha}$ é limitada em $\left[t_{0}, t_{1}\right)$, de onde segue o resultado.

O próximo resultado é uma alternativa ao corolário anterior, uma vez que ele também fornece condições para que o mesmo problema de Cauchy tenha solução global. Para a demonstração, 
remetemos o leitor a [23].

Teorema 29 Sejam $X$ um espaço de Banach e $f:[0, \infty) \times X \longrightarrow X$ uma função contínua em $t$ para $t \geq 0$ e localmente lipschiztiana na segunda variável. Se A for um operador setorial em $X$ então, para cada $u_{0} \in X$, existe um $t_{\text {max }} \leq \infty$ tal que o problema de valor inicial 3.32 tem uma única solução $u$ em $\left[0, t_{\max }\right)$. Além disso, se $t_{\max }<\infty$ então $\lim _{t \rightarrow t_{\max }}\|u(t)\|=\infty$. 


\section{Capítulo 4}

\section{Sistemas de reação-difusão: aplicações de semigrupos, comparação e ondas viajantes}

O foco deste capítulo é discutir aspectos do modelo de reação-difusão que foi introduzido anteriormente, uma vez que todos os elementos necessários para tal discussão já nos foram apresentados no capítulo anterior. O primeiro passo será o estabelecimento de um teorema de existência e unicidade no contexto do modelo 2.30 e a aplicação deste mesmo teorema a um particular modelo, que é um caso especial de uma classe de equações conhecida como $\lambda-\omega$. Passaremos em seguida a uma discussão acerca de uma outra particular formulação, conhecida como modelo de RosenzweigMacArthur, no contexto do qual aplicaremos um teorema comparação afim de concluirmos a existência e unicidade e, mais ainda, discutiremos a noção de bifurcação sob a perspectiva do teorema devido a Hopf com a finalidade de concluirmos a existência de soluções do tipo ondas viajantes para este particular modelo.

\subsection{Existência e unicidade para o modelo envolvendo populações cíclicas}

Inúmeros estudos têm se preocupado com aspectos envolvendo a densidade de populações com ciclos pluri-anuais ${ }^{1}$. Isto se deve ao fato de que tais populações apresentam um particular tipo de distribuição que é não uniforme e na qual a densidade varia periodicamente em uma direção espacial e também no tempo, de modo que quando combinadas, aparentam uma onda. Dado então um modelo envolvendo a densidade de tais populações, é natural esperar que a solução seja do tipo ondas viajantes. Portanto, nosso objetivo será considerar um sistema que envolve a densidade de duas espécies que interagem, impor condições acerca das variáveis do modelo de modo que ele possua uma única solução e então veremos, na seção seguinte, que tal solução deve ser do tipo ondas viajantes.

Sejam $u:[0, \infty) \times \mathbb{R}^{2} \longrightarrow \mathbb{R}$ e $v:[0, \infty) \times \mathbb{R}^{2} \longrightarrow \mathbb{R}$ as densidades de duas espécies que interagem de alguma maneira, então um sistema de reação-difusão simples que modela este tipo de situação é dado por

$$
\begin{gathered}
u_{t}=D_{1} \Delta u+f_{1}(u, v) \\
v_{t}=D_{2} \Delta v+f_{2}(u, v),
\end{gathered}
$$

\footnotetext{
${ }^{1}$ Ver [16]
} 
onde $D_{1}, D_{2}$ são constantes, $t$ denota tempo e $\Delta$ indica o laplaciano em relação às coordenadas espaciais, ou seja, $\Delta=\frac{\partial^{2}}{\partial x^{2}}+\frac{\partial^{2}}{\partial y^{2}}$. Veremos ainda, na seção 4.2 , que, a fim de que tal sistema apresente soluções de ondas viajantes periódicas, deve-se assumir que, dado um espaço de funções $W$ arbitrário a princípio e um subconjunto $U \in W \times W$, as dinâmicas locais de $f_{1}: U \longrightarrow W$ e $f_{2}: U \longrightarrow W$ são tais que as equações espacialmente uniformes

$$
u_{t}=f_{1}(u, v) \text { e } v_{t}=f_{2}(u, v)
$$

possuam soluções periódicas estáveis que correpondam, na verdade, a existência de ciclo limite e que oscilem ao redor de um equilíbrio estável ${ }^{2}$. Adicionamos ao sistema 4.1 as seguintes condições iniciais

$$
\begin{aligned}
& u(0)=u_{0} \\
& v(0)=v_{0},
\end{aligned}
$$

onde $u_{0}$ e $v_{0}$ são funções a princípio arbitrárias definidas em $\mathbb{R}^{2}$.

Podemos assumir, apenas por uma questão de conveniência que $D_{1}=D_{2}=1$ até obtermos o resultado de existência e unicidade para 4.1- 4.3, que por sua vez será válido para quaisquer constantes $D_{1}$ e $D_{2}$. Uma vez que nosso objetivo é aplicar os resultados obtidos no capítulo anterior, devemos escrever o sistema 4.1- 4.3 da seguinte forma

$$
\left\{\begin{array}{c}
z_{t}=-A z+f(z), \quad \forall t>0 \\
z(0)=z_{0},
\end{array}\right.
$$

onde $A$ deve ser um operador linear fechado e densamente definido em um espaço de Banach $X$ e $f$ localmente lipschitziana em $z$.

Consideremos então o espaço de Banach $Y=L_{2}\left(\mathbb{R}^{2} ; \mathbb{R}\right)=L_{2}\left(\mathbb{R}^{2}\right)$ e o operador $B_{0}: D\left(B_{0}\right) \subset$ $Y \longrightarrow Y$ dado por $B_{0} u=-\Delta u$, onde $D\left(B_{0}\right)=C_{c}^{\infty}\left(\mathbb{R}^{2}\right)$ é formado pelo conjunto das funções definidas em $\mathbb{R}$ tomando valores em $\mathbb{R}$ que são infinitamente continuamente diferenciáveis com suporte compacto.

Veremos que $B_{0}$ é um operador fechável e definiremos $B: D(B) \in Y \longrightarrow Y$ como sendo seu fecho em $L_{2}\left(\mathbb{R}^{2}\right)$, ou seja, $B$ será o operador linear cujo gráfico $\{(u, B u) ; u \in D(B)\}$ é o fecho em $L_{2}\left(\mathbb{R}^{2}\right) \times L_{2}\left(\mathbb{R}^{2}\right)$ do gráfico de $B_{0}$. Assim definido, $B$ será um operador linear fechado e densamente definido.

Apresentaremos a seguir um resultado que afirma que $B_{0}$ é de fato um operador fechável em $L_{2}\left(\mathbb{R}^{2}\right)$. Mais ainda, obteremos o espaço $D(B)$ onde o fecho de $B_{0}$ estará definido. Uma vez que a demonstração envolve uma estimativa, conhecida como desigualdade do tipo Calderón-Zygmund, segue a princípio um teorema com este conteúdo e, cuja prova pode ser encontrada em [22].

\footnotetext{
${ }^{2}$ Tal propriedade deve ser verificada neste contexto pelo fato de esta ser umas das condições necessárias para que a existência de ondas viajantes periódicas seja garantida, como veremos na próxima seção.
} 
Teorema 30 Seja $\Omega \subset \mathbb{R}^{n}$ um conjunto não vazio e limitado com fronteira $\partial \Omega$ de classe $C^{2}$. Então, existe uma constante $C>0$ tal que

$$
\|u\|_{H^{2}(\Omega)} \leq C\left(\|\Delta u\|_{L^{2}(\Omega)}+\|u\|_{L^{2}}(\Omega)\right)
$$

para toda função $u \in H^{2}(\Omega) \cap H_{0}^{1}(\Omega)$.

Lema 15 operador laplaciano $\Delta: C_{c}^{\infty}\left(\mathbb{R}^{n}\right) \longrightarrow L_{2}\left(\mathbb{R}^{n}\right)$ é fechável em $L_{2}\left(\mathbb{R}^{n}\right)$. Além disso, o domínio do fecho de $\Delta$ em $L_{2}\left(\mathbb{R}^{n}\right)$, que denotaremos por $\Delta_{D}$, coincide com o espaço $H^{2}\left(\mathbb{R}^{n}\right)$, ou seja, $D\left(\Delta_{D}\right)=H^{2}\left(\mathbb{R}^{n}\right)$.

Demonstração. Seja $G(\Delta)$ o gráfico de $\Delta: C_{c}^{\infty}\left(\mathbb{R}^{n}\right) \longrightarrow L_{2}\left(\mathbb{R}^{n}\right)$ em $L_{2}\left(\mathbb{R}^{n}\right) \times L_{2}\left(\mathbb{R}^{n}\right)$. Queremos verificar que o fecho de $G(\Delta)$ coincide com o gráfico de um operador que é uma extensão de $\Delta$ e, ainda, descrever o domínio de tal operador.

Veremos a princípio que a seguinte desigualdade, do tipo Calderón-Zygmund, se verifica

$$
\frac{1}{\sqrt{1+n+n^{2}}}\|u\|_{H^{2}}\left(\mathbb{R}^{n}\right) \leq\|u\|_{L_{2}\left(\mathbb{R}^{n}\right)}+\|\Delta u\|_{L_{2}\left(\mathbb{R}^{n}\right)} \leq \sqrt{n+1}\|u\|_{H^{2}\left(\mathbb{R}^{n}\right)},
$$

para todo $u \in C_{c}^{\infty}\left(\mathbb{R}^{n}\right)$. De fato, observamos que

$$
\begin{aligned}
& \|u\|_{L_{2}\left(\mathbb{R}^{n}\right)}+\|\Delta u\|_{L_{2}\left(\mathbb{R}^{n}\right)} \leq\|u\|_{L_{2}\left(\mathbb{R}^{n}\right)}+\sum_{i=1}^{n}\left\|\frac{\partial^{2} u}{\partial x_{i}^{2}}\right\|_{L_{2}\left(\mathbb{R}^{n}\right)} \\
& \quad \leq \frac{n+1}{\sqrt{n+1}} \sqrt{\|u\|_{L_{2}\left(\mathbb{R}^{n}\right)}^{2}+\sum_{i=1}^{n}\left\|\frac{\partial^{2} u}{\partial x_{i}^{2}}\right\|_{L_{2}\left(\mathbb{R}^{n}\right)}^{2}} \\
& \quad \leq \sqrt{n+1}\|u\|_{H^{2}\left(\mathbb{R}^{n}\right)} .
\end{aligned}
$$

Para provarmos a primeira desigualdade em 4.5 observamos inicialmente que a transformada de Fourier $\mathcal{F}$ é um isomorfismo em $L_{2}\left(\mathbb{R}^{n}\right)$ e, portanto, uma isometria nesse mesmo espaço. Notemos ainda que

$$
\mathcal{F} \frac{\partial^{2} u(\xi)}{\partial x_{j} \partial x_{k}}=-\xi_{j} \xi_{k} \mathcal{F} u(\xi) \text { e } \mathcal{F}[(I-\Delta) u](\xi)=\left(1+|\xi|^{2}\right) \mathcal{F} u(\xi),
$$

para $j, k \in\{1,2, \cdots, n\}$ e $u \in C_{c}^{\infty}\left(\mathbb{R}^{n}\right)$. De onde obtemos as seguintes desigualdades, para toda função $u \in C_{c}^{\infty}\left(\mathbb{R}^{n}\right)$,

$$
\begin{aligned}
& \left\|\frac{\partial^{2} u}{\partial x_{j} \partial x_{k}}\right\|_{L_{2}}=\left\|\mathcal{F} \frac{\partial^{2} u}{\partial x_{j} \partial x_{k}}\right\|_{L_{2}}=\left\|-\xi_{j} \xi_{k} \mathcal{F} u\right\|_{L_{2}\left(\mathbb{R}^{n}\right)} \\
& \quad \leq\left\|\left(1+|\xi|^{2}\right) \mathcal{F} u\right\|_{L_{2}\left(\mathbb{R}^{n}\right)}=\|\mathcal{F}[(I-\Delta) u]\|_{L_{2}\left(\mathbb{R}^{n}\right)} \\
& \quad=\|(I-\Delta) u\|_{L_{2}\left(\mathbb{R}^{n}\right)}
\end{aligned}
$$


e

$$
\begin{aligned}
& \left\|\frac{\partial u}{\partial x_{j}}\right\|_{L^{2}\left(\mathbb{R}^{n}\right)}=\left\|\mathcal{F} \frac{\partial u}{\partial x_{j}}\right\|_{L^{2}\left(\mathbb{R}^{n}\right)}=\left\|i \xi_{j} \mathcal{F} u\right\|_{L^{2}\left(\mathbb{R}^{n}\right)} \\
& \quad \leq\left\|\left(1+|\xi|^{2}\right) \mathcal{F} u\right\|_{L_{2}\left(\mathbb{R}^{n}\right)}=\|\mathcal{F}[(I-\Delta) u]\|_{L_{2}\left(\mathbb{R}^{n}\right)} \\
& \quad=\|(I-\Delta) u\|_{L_{2}\left(\mathbb{R}^{n}\right)} .
\end{aligned}
$$

Finalmente, é possível verificar que

$$
\begin{aligned}
& \|u\|_{H^{2}\left(\mathbb{R}^{n}\right)}^{2} \leq\|u\|_{L_{2}\left(\mathbb{R}^{n}\right)}^{2}+n\|(I-\Delta) u\|_{L_{2}\left(\mathbb{R}^{n}\right)}^{2}+n^{2}\|(I-\Delta) u\|_{L_{2}\left(\mathbb{R}^{n}\right)}^{2} \\
& \quad \leq\|u\|_{L_{2}\left(\mathbb{R}^{n}\right)}+\left(n+n^{2}\right)\left(\|u\|_{L_{2}\left(\mathbb{R}^{n}\right)}+\|\Delta u\|_{L_{2}\left(\mathbb{R}^{n}\right)}\right)^{2} \\
& \quad \leq\left(1+n+n^{2}\right)\left(\|u\|_{L_{2}\left(\mathbb{R}^{n}\right)}+\|\Delta u\|_{L_{2}\left(\mathbb{R}^{n}\right)}\right)^{2}
\end{aligned}
$$

de onde segue a estimativa 4.5 .

Considere uma seqüência $\left\{u_{m}\right\}_{m \in \mathbb{N}}$ em $C_{c}^{\infty}\left(\mathbb{R}^{n}\right)$ tal que

$$
u_{m} \longrightarrow 0 \text { e } \Delta u_{m} \longrightarrow v \text {, se } m \rightarrow \infty,
$$

em $L_{2}\left(\mathbb{R}^{n}\right)$. O primeira desigualdade em 4.5 nos permite concluir que $\left\{u_{m}\right\}_{m \in \mathbb{N}}$ é de Cauchy em $H^{2}\left(\mathbb{R}^{n}\right)$, que é um espaço completo com a norma $\|\cdot\|_{H^{2}\left(\mathbb{R}^{n}\right)}$. Portanto, existe $u \in H^{2}\left(\mathbb{R}^{n}\right) \subset L_{2}\left(\mathbb{R}^{n}\right)$ tal que $u_{m} \longrightarrow u$ na norma de $H^{2}\left(\mathbb{R}^{n}\right)$. Uma vez que

$$
0 \leq\left\|u_{m}-u\right\|_{L_{2}\left(\mathbb{R}^{n}\right)} \leq\left\|u_{m}-u\right\|_{H^{2}\left(\mathbb{R}^{n}\right)}, \quad \forall m \in \mathbb{N}
$$

a sequiência $\left\{u_{m}\right\}_{m \in \mathbb{N}}$ converge para $u$ em $L_{2}\left(\mathbb{R}^{n}\right)$. De onde obtemos

$$
u \equiv 0 \text { e } u_{m} \longrightarrow u, \text { quando } m \rightarrow \infty
$$

na norma de $H^{2}\left(\mathbb{R}^{n}\right)$.

Por outro lado, é possível obter, a partir das conclusões acima e da segunda desigualdade em 4.5, que

$$
\Delta u_{m} \longrightarrow 0, \text { quando } m \rightarrow \infty,
$$

na norma de $L_{2}\left(\mathbb{R}^{n}\right)$. Portanto, $v \equiv 0$ e assim, segue da proposição 1 que $\Delta: C_{c}^{\infty}\left(\mathbb{R}^{n}\right) \longrightarrow L_{2}\left(\mathbb{R}^{n}\right)$ é um operador fechável em $L_{2}\left(\mathbb{R}^{n}\right)$.

Denotaremos por $\Delta_{D}$ o fecho de $\Delta$ em $L_{2}\left(\mathbb{R}^{n}\right)$. Nosso próximo passo é encontrar o domínio de $\Delta_{D}, D\left(\Delta_{D}\right)$. Considere então um elemento $(u, v) \in \overline{G(\Delta)}$, onde $\overline{G(\Delta)}$ denota o fecho de $G(\Delta)$ em $L_{2}\left(\mathbb{R}^{n}\right) \times L_{2}\left(\mathbb{R}^{n}\right)$. Para algum $\left(u_{m}, \Delta u_{m}\right) \in G(\Delta)$ deve-se ter

$$
u_{m} \longrightarrow \text { u e } \Delta u_{m} \longrightarrow v \text {, quando } m \rightarrow \infty
$$


na norma de $L_{2}\left(\mathbb{R}^{n}\right)$. Mais uma vez, a primeira desigualdade em 4.5 implica que $\left\{u_{m}\right\}_{m \in \mathbb{N}}$ é uma seqüência de Cauchy em $H^{2}\left(\mathbb{R}^{n}\right)$ e, portanto, deve convergir para um ponto desse mesmo espaço. De onde obtemos que $u \in H^{2}\left(\mathbb{R}^{n}\right)$, o que mostra que $D\left(\Delta_{D}\right) \subset H^{2}\left(\mathbb{R}^{n}\right)$. Para justificar a inclusão contrária, consideramos um elemento $u \in H^{2}\left(\mathbb{R}^{n}\right)$ e uma seqüência $\left\{u_{m}\right\}_{m \in \mathbb{N}}$ em $C_{c}^{\infty}\left(\mathbb{R}^{n}\right)$ tais que

$$
u_{m} \longrightarrow u, \text { quando } m \rightarrow \infty
$$

na norma de $H^{2}\left(\mathbb{R}^{n}\right)$. A segunda desigualdade em 4.5 nos garante que $\left\{\Delta u_{m}\right\}_{m \in \mathbb{N}}$ é uma seqüência de Cauchy em $L_{2}\left(\mathbb{R}^{n}\right)$ e, portanto, deve existir $v \in L_{2}\left(\mathbb{R}^{n}\right)$ tal que

$$
\Delta u_{m} \longrightarrow v, \text { quando } m \rightarrow \infty
$$

na norma de $L_{2}\left(\mathbb{R}^{n}\right)$. Obtemos com isso,

$$
\left(u_{m}, \Delta u_{m}\right) \longrightarrow(u, v), \text { quando } m \rightarrow \infty
$$

na norma de $L_{2}\left(\mathbb{R}^{n}\right) \times L_{2}\left(\mathbb{R}^{n}\right)$, o que implica que $(u, v) \in \overline{G(\Delta)}$, ou seja, $u \in D\left(\Delta_{D}\right)$.

Reunindo os argumentos acima, concluímos que $H^{2}\left(\mathbb{R}^{n}\right)$ é o domínio do fecho de

$$
\Delta: C_{c}^{2}\left(\mathbb{R}^{n}\right) \longrightarrow L_{2}\left(\mathbb{R}^{n}\right)
$$

Definimos então $A$ como sendo o operador diagonal

$$
A=\left(\begin{array}{cc}
-\Delta & 0 \\
0 & -\Delta
\end{array}\right) \text {, }
$$

mas, por uma questão de conveniência, iremos considerar $A$ como um operador da seguinte forma $A: D(A) \subset X \longrightarrow X$ onde $A=B \times B$, onde $X=Y \times Y$. Mais precisamente, $A: D(A) \in Y \times Y \longrightarrow$ $Y \times Y$ é o operador dado por $A(u, v)=(B u, B v)$ para todo $(u, v) \in D(A)=D(B) \times D(B)$. Portanto, $A$ é um operador linear fechado e densamente definido no espaço de $\operatorname{Banach}^{3} L_{2}\left(\mathbb{R}^{2}\right) \times L_{2}\left(\mathbb{R}^{2}\right)$.

Nosso próximo passo será provar que o operador linear $A$, como foi definido acima, é um operador setorial. Caso esta afirmação se verifique, estarão bem definidas as potências fracionárias do operador $A_{1}=A+I$ e poderemos então encontrar o espaço $X^{\alpha}=D\left(A_{1}^{\alpha}\right)$ que, como veremos, irá coincidir com um particular espaço de potências de Bessel. Se, além disso, $f$ for uma função definida em um aberto $U \in X^{\alpha} \times X^{\alpha}$, para algum $\alpha \in[0,1)$, tomando valores em $X$, poderemos então aplicar alguns resultados da subseção 3.4 e concluirmos aspectos das soluções do modelo de reação-difusão 4.4 e, como conseqüência, do sistema 4.1- 4.3.

Veremos agora que o operador $A$ é de fato um operador setorial. De acordo com a proposição 4

\footnotetext{
${ }^{3}$ Estamos considerando o produto cartesiano destes dois espaços com a topologia induzida pelo produto, o que preserva a noção de completude, ou seja, $Y \times Y$ é também um espaço de Banach.
} 
é suficiente provarmos que $B=-\Delta$ é setorial em $L_{2}(\mathbb{R})$. Como vimos $B$ é o operador

$$
-\Delta: H^{2}\left(\mathbb{R}^{2}\right) \longrightarrow L_{2}\left(\mathbb{R}^{2}\right) .
$$

O próximo resultado mostra que $I+B$ é um operador setorial ${ }^{4}$, de onde é possível obter que $B$ é também um operador setorial, uma vez que $\rho(I+B)=\rho(B)$.

Lema 16 operador $I-\Delta: H^{2}\left(\mathbb{R}^{n}\right) \subset L_{2}\left(\mathbb{R}^{n}\right) \longrightarrow L_{2}\left(\mathbb{R}^{n}\right)$ é setorial.

Demonstração. Sendo $L_{2}\left(\mathbb{R}^{n}\right)$ um espaço de Hilbert, é suficiente provarmos, de acordo com o teorema 16, que $I-\Delta$ é auto-adjunto e que existe uma constante $C$ tal que

$$
\langle(I-\Delta) u, u\rangle_{L_{2}\left(\mathbb{R}^{n}\right)} \geq C\|u\|_{L_{2}\left(\mathbb{R}^{n}\right)}^{2}, \quad \forall u \in H^{2}\left(\mathbb{R}^{n}\right) .
$$

Veremos inicialmente que $I-\Delta$ é simétrico e satisfaz 4.6. Considere então duas funções $u, v \in$ $C_{c}^{\infty}\left(\mathbb{R}^{n}\right)$. Escolhendo $r>0$ de modo que $u(x)=v(x)=0$ para todo $x \notin B(0, r)$, obtemos, integrando por partes duas vezes,

$$
\begin{aligned}
\int_{\mathbb{R}^{n}} & {[(I-\Delta) u] \overline{v(x)} d x=\int_{\mathbb{R}^{n}} u(x) \overline{v(x)} d x-\int_{B(0, r)} \Delta u(x) \overline{v(x)} d x } \\
= & \left.\int_{\mathbb{R}^{n}} u(x) \overline{v(x)} d x-\int_{B(0, r)} u(x) \overline{\Delta v(x)} d x=\int_{\mathbb{R}^{n}} u(x) \overline{[(I-\Delta) v(x)}\right] d x,
\end{aligned}
$$

ou seja,

$$
\langle(I-\Delta) u, v\rangle_{L_{2}\left(\mathbb{R}^{n}\right)}=\langle u,(I-\Delta) v\rangle_{L_{2}\left(\mathbb{R}^{n}\right)}, \quad \forall u, v \in C_{c}^{2}\left(\mathbb{R}^{n}\right) .
$$

Ainda integrando por partes, obtemos

$$
\begin{gathered}
\int_{\mathbb{R}^{n}}[(I-\Delta) u(x)] \overline{u(x)} d x=\int_{\mathbb{R}^{n}}|u(x)|^{2} d x-\int_{B(0, r)} \Delta u(x) \overline{u(x)} d x \\
=\int_{\mathbb{R}^{n}}|u(x)|^{2} d x+\int_{B(0, r)}|\nabla u(x)|^{2} d x \geq \int_{\mathbb{R}^{n}}|u(x)|^{2} d x,
\end{gathered}
$$

onde $\nabla=\left(\frac{\partial}{\partial x_{1}}, \cdots, \frac{\partial}{\partial x_{n}}\right)$. De onde segue que

$$
\langle(I-\Delta) u, u\rangle_{L_{2}\left(\mathbb{R}^{n}\right)} \geq\|u\|_{L_{2}\left(\mathbb{R}^{n}\right)}^{2}, \quad \forall u \in C_{c}^{2}\left(\mathbb{R}^{n}\right) .
$$

Sendo $C_{c}^{\infty}\left(\mathbb{R}^{n}\right)$ um subespaço denso em $L_{2}\left(\mathbb{R}^{n}\right)$, concluímos, das afirmações 4.7 e 4.8 que $I-\Delta$ é de fato um operador simétrico que satisfaz 4.6, com constante $C=1$.

Nosso próximo passo é provar que a imagem de $I-\Delta, R(I-\Delta)$, coincide com o espaço $L_{2}\left(\mathbb{R}^{n}\right)$, uma vez que o lema 5 nos garante que tal condição é suficiente para afirmarmos que $I-\Delta$ é

\footnotetext{
${ }^{4} \mathrm{O}$ resultado apresentado em seguida, assim como o lema anterior, podem ser encontrados em [9]. Tal texto apresenta uma exposição detalhada acerca das equações envolvendo operadores setoriais.
} 
auto-adjunto. Afirmamos inicialmente que $R(I-\Delta)$ é um subconjunto denso em $L_{2}\left(\mathbb{R}^{n}\right)$, já que tal subconjunto contém o espaço $C_{c}^{\infty}\left(\mathbb{R}^{n}\right)$, que é denso em $L_{2}\left(\mathbb{R}^{n}\right)$. De fato, considerando um elemento $u$ de $C_{c}^{\infty}\left(\mathbb{R}^{n}\right)$, que por sua vez está contido em $S$, podemos definir

$$
v(x)=\mathcal{F}^{-1}\left(\frac{1}{1+|\xi|^{2}} \mathcal{F} u(\xi)\right)(x)
$$

pois a transformada de Fourier $\mathcal{F}: S \longrightarrow S$ é um isomorfismo. Assim sendo, $v \in S$ e

$$
\mathcal{F}[(I-\Delta) v](\xi)=\mathcal{F} v(\xi)-\mathcal{F} \Delta v(\xi)=\frac{1}{1+|\xi|^{2}} \mathcal{F} u(\xi)+|\xi|^{2} \mathcal{F} v(\xi)=\mathcal{F} u(\xi),
$$

para todo $\xi \in \mathbb{R}^{n}$. Aplicando $\mathcal{F}^{-1}$, obtemos $(I-\Delta) v=u$, ou seja, $u \in R(I-\Delta)$. Portanto, segue da densidade de $C_{c}^{\infty}\left(\mathbb{R}^{n}\right)$ em $L_{2}\left(\mathbb{R}^{n}\right)$, que a imagem de $I-\Delta$ é densa em $L_{2}\left(\mathbb{R}^{n}\right)$.

Obtemos ainda, da estimativa 4.8, da desigualdade de Schwartz e da densidade de $C_{c}^{2}\left(\mathbb{R}^{n}\right)$ em $H^{2}\left(\mathbb{R}^{n}\right)$ que

$$
\|(I-\Delta) u\|_{L_{2}\left(\mathbb{R}^{n}\right)} \geq\|u\|_{L_{2}\left(\mathbb{R}^{n}\right)},
$$

para toda função $u \in H^{2}\left(\mathbb{R}^{n}\right)$. De onde segue que o operador linear $(I-\Delta)^{-1}: R(I-\Delta) \longrightarrow H^{2}\left(\mathbb{R}^{n}\right)$ existe e é limitado em $L_{2}\left(\mathbb{R}^{n}\right)$. Aplicando o lema anterior e usando o fato de $A$ ser um operador fechado, concluímos que $(I-\Delta)^{-1} \in \mathcal{L}\left(L_{2}\left(\mathbb{R}^{n}\right)\right)$ e, em particular, que $R(I-\Delta)=L_{2}\left(\mathbb{R}^{n}\right)$.

Finalmente, obtemos do teorema 16 , que $I-\Delta: H^{2}\left(\mathbb{R}^{n}\right) \subset L_{2}\left(\mathbb{R}^{n}\right) \longrightarrow L_{2}\left(\mathbb{R}^{n}\right)$ é um operador setorial.

Sendo $A$ um operador setorial, o espaço $X^{\alpha}=D\left(A_{1}^{\alpha}\right)$ está bem definido. Vamos então determina-lo. Observamos a princípio que as potências de Bessel $\mathcal{J}_{\alpha}$ são definidas por

$$
(I-\Delta)^{-\frac{\alpha}{2}}
$$

e, cada função $f \in L_{2}\left(\mathbb{R}^{n}\right)$ pode ser representada, em termos de $\mathcal{J}_{\alpha}$, da seguinte forma

$$
\mathcal{J}_{\alpha}(f)=G_{\alpha} * f
$$

onde

$$
G_{\alpha}(x)=\frac{1}{(4 \pi)^{\frac{n}{2}}} \frac{1}{\Gamma\left(\frac{\alpha}{2}\right)} \int_{0}^{\infty} t^{\frac{\alpha-n}{2}} e^{-\frac{\pi|x|^{2}}{t}} e^{-\frac{t}{4 \pi}} \frac{d t}{t} .
$$

De onde segue que $D\left[(I-\Delta)^{\frac{\alpha}{2}}\right]=\left\{G_{\alpha} * f: f \in L_{2}\left(\mathbb{R}^{n}\right)\right\}=\mathcal{L}_{\alpha}^{2}\left(\mathbb{R}^{n}\right)$ e, portanto, de acordo com o teorema 35 no apêndice deste trabalho,

$$
\left.D[(I+B)]^{\alpha}\right)=D\left[(I-\Delta)^{\alpha}\right]=\mathcal{L}_{2 \alpha}^{2}\left(\mathbb{R}^{n}\right)=H^{2 \alpha} .
$$

Logo, $D\left(A_{1}^{\alpha}\right)=H^{2 \alpha} \times H^{2 \alpha}$.

\footnotetext{
${ }^{5}$ Para uma breve discussão acerca deste tema ver apêndice deste trabalho. Já para uma exposição mais detalhada sugerimos o texto [38]
} 
Provamos então, como conseqüência do teorema 27 para $f(u, v)=\left(f_{1}(u, v), f_{2}(u, v)\right)$ e $A$ como foi definido acima, o seguinte

Corolário 5 Sejam $u, v:[0, \infty) \times \mathbb{R}^{2} \longrightarrow \mathbb{R}$ e $f_{1}, f_{2}: U \subset H^{2}\left(\mathbb{R}^{2}\right) \times H^{2}\left(\mathbb{R}^{2}\right) \longrightarrow L_{2}\left(\mathbb{R}^{2}\right)$, onde $U$ é um subconjunto aberto. Se, além disso, u e v são tais que $u(t), v(t) \in H^{2}\left(\mathbb{R}^{2}\right)$ para todo $t>0$ e $f_{1}, f_{2}$ são funções localmente lipschitzianas, então, para cada $\left(u_{0}, v_{0}\right) \in U$, o sistema

$$
\begin{aligned}
& u_{t}=D_{1} \Delta u+f_{1}(u, v) \\
& v_{t}=D_{2} \Delta v+f_{2}(u, v) \\
& u(0)=u_{0}, \quad v(0)=v_{0}
\end{aligned}
$$

tem uma única solução $(u, v)$ em $(0, \tau) \operatorname{com} \tau=\tau\left(u_{0}, v_{0}\right)$.

O próximo resultado garante ainda a existência global de uma solução de 4.9 e é uma conseqüência do corolário 4.

Corolário 6 Sejam u,v : $[0, \infty) \times \mathbb{R}^{2} \longrightarrow \mathbb{R}$ e $f_{1}, f_{2}: U \subset H^{2}\left(\mathbb{R}^{2}\right) \times H^{2}\left(\mathbb{R}^{2}\right) \longrightarrow L_{2}\left(\mathbb{R}^{2}\right)$, onde $U$ é um subconjunto aberto. Se, além disso, u e $v$ são tais que $u(t), v(t) \in H^{2}\left(\mathbb{R}^{2}\right)$ para todo $t>0$ e $f_{1}, f_{2}$ são funções localmente lipschitzianas em $U$ e existe ainda uma função contínua não-decrescente $K:(0, \infty) \longrightarrow \mathbb{R}$ tal que

$$
\left\|\left(f_{1}(u, v), f_{2}(u, v)\right)\right\| \leq K(t)\left(1+\|(u, v)\|_{H^{2}\left(\mathbb{R}^{2}\right) \times H^{2}\left(\mathbb{R}^{2}\right)}\right), \quad \forall t \geq 0 e(u, v) \in U \times U
$$

então, para cada $\left(u_{0}, v_{0}\right) \in H^{2}\left(\mathbb{R}^{2}\right) \times H^{2}\left(\mathbb{R}^{2}\right)$, a única solução do sistema 4.9 existe para todo $t \geq 0$.

Para ilustrar os resultados acima apresentaremos um particular modelo, que é um caso especial de uma classe de equações conhecida como $\lambda-\omega$, e nosso objetivo é concluir existência e unicidade de soluções impondo condições iniciais.

O modelo que pretendemos estudar é um caso particular de uma classe de equações introduzidas por Kopell e Howard (ver [15]) e é dado por

$$
\begin{aligned}
& u_{t}=u_{x x}+u_{y y}+\left(1-u^{2}-v^{2}\right) u-\left(\omega_{0}-\omega_{1} u^{2}-\omega_{1} v^{2}\right) v \\
& v_{t}=v_{x x}+v_{y y}+\left(\omega_{0}-\omega_{1} u^{2}-\omega_{1} v^{2}\right) u+\left(1-u^{2}-v^{2}\right) v
\end{aligned}
$$

onde $\omega_{0}$ e $\omega_{1}$ são constantes que devem satisfazer $\omega_{0}\left(\omega_{0}-\omega_{1}\right)>0$. Adicionando ao sistema acima as seguintes condições iniciais

$$
u(0)=u_{0}, \quad v(0)=v_{0},
$$

e definindo as funções

$$
f_{1}(u, v)=\left(1-u^{2}-v^{2}\right) u-\left(\omega_{0}-\omega_{1} u^{2}-\omega_{1} v^{2}\right) v
$$


e

$$
f_{2}(u, v)=\left(\omega_{0}-\omega_{1} u^{2}-\omega_{1} v^{2}\right) u+\left(1-u^{2}-v^{2}\right) v
$$

o corolário 5 pode ser aplicado diretamente ao problema de Cauchy 4.11-4.12. De fato, as nãolinearidades $f_{1}$ e $f_{2}$ são de classe $C^{1}$ e, portanto, localmente lipschtizianas em $H^{2}\left(\mathbb{R}^{2}\right) \times H^{2}\left(\mathbb{R}^{2}\right)$.

\subsection{O modelo de Rosenzweig-MacArthur e a existência de ondas viajantes periódicas}

Estudaremos nesta seção, como um outro exemplo concreto de 4.1- 4.3, um sistema para interações do tipo predador-presa, conhecido como modelo de Rosenzweig-MacArthur. Aplicaremos um resultado de comparação devido a C.V. Pao que será apresentado adiante, a fim de discutirmos questões como existência e unicidade de soluções. Mais ainda, iremos descrever condições a serem impostas, de modo que este mesmo sistema tenha soluções do tipo ondas viajantes. Usaremos como ferramenta um teorema de bifurcação de Hopf, uma vez que este nos fornece condições para a existência de ciclos limites que, como vimos no capítulo 2, correspondem a ondas viajantes periódicas.

Dadas duas populações com densidades $u$ e $v$, o modelo de Rosenzweig-MacArthur pode ser escrito da seguinte forma

$$
\begin{gathered}
u_{t}=\frac{1}{\sqrt{\alpha}}\left(u_{x x}+u_{y y}\right)+\frac{\sigma u v}{k+v}-\mu u, \\
v_{t}=\sqrt{\alpha}\left(u_{x x}+u_{y y}\right)+v(1-v)-\frac{u v}{k+v},
\end{gathered}
$$

onde $\alpha, \sigma, k$ e $\mu$ são constantes. Uma vez que o foco deste trabalho é a aplicação de um instrumental matemático, não ofereceremos a interpretação do modelo acima. O leitor interessado no significado biológico dos parametros pode consultar os textos [19] e [16].

Definiremos inicialmente as funções

$$
f_{1}(u, v)=\frac{\sigma u v}{k+v}-\mu u \text { e } f_{2}(u, v)=v(1-v)-\frac{u v}{k+v}
$$

cujo domínio será o espaço de Banach $H^{2}\left(\mathbb{R}^{2}\right) \times H^{2}\left(\mathbb{R}^{2}\right)$. Sendo assim, é possível obter, graças às imersões encontradas no teorema de Sobolev $^{6}$ que, as funções $f_{1}$ e $f_{2}$ assim definidas, tomam valores em $L_{2}\left(\mathbb{R}^{2}\right)$. Observamos que os resultados obtidos na seção anterior não se aplicam, pelo menos não diretamente, ao modelo 4.13. Isto se deve ao fato de que tais não-linearidades não são sequer contínuas em todo o espaço $H^{2}\left(\mathbb{R}^{2}\right) \times H^{2}\left(\mathbb{R}^{2}\right)$. Sendo assim, não é possível obter existência e unicidade de soluções para qualquer condição inicial $\left(u_{0}, v_{0}\right) \in H^{2}\left(\mathbb{R}^{2}\right) \times H^{2}\left(\mathbb{R}^{2}\right)$. Além disso, o corolário 6 são se aplica para esse caso, já que $f_{1}$ e $f_{2}$ não satizafem 4.10 para nenhuma função não-decrescente $K$.

Uma solução seria encontrar uma região em $H^{2}\left(\mathbb{R}^{2}\right) \times H^{2}\left(\mathbb{R}^{2}\right)$ que fosse invariante pelo sistema 4.13, ou seja, uma região $R$ onde qualquer solução com condições iniciais em $R$ permaneça

\footnotetext{
${ }^{6} \mathrm{O}$ teorema de Sobolev ao qual estamos nos referindo pode ser encontrado no apêndice deste trabalho
} 
neste mesmo conjunto para qualquer tempo $t$ para o qual esteja definida. Além disso, tal região $R$ deveria ser tal que as não linearidades fossem localmente lipschitzianas quando restritas a ela. Uma vez encontrada uma região com tais propriedades, poderíamos aplicar o corolário 5 para concluir a existência e unicidade local de soluções e, mais ainda, concluir a existência global, uma vez que sendo limitadas, tais soluções devem estar definidas para todo $t>0$ (ver teorema 29).

Porém o que nos propomos a fazer é aplicar um resultado que trata de sistemas de reaçãodifusão em domínios ilimitados e nos garante as mesmas conclusões acima para o modelo 4.13. Uma caracteristica fundamental que difere tal resultado do corolário 5 envolve a noção de solução. Ao passo que o corolário se preocupa com soluções definidas em $H^{2}\left(\mathbb{R}^{2}\right) \times H^{2}\left(\mathbb{R}^{2}\right)$, o resultado devido a Pao está interessado apenas em soluções contínuas - de acordo com a definição 39. Já que nosso objetivo é procurar por soluções de ondas viajantes, que é um tipo de solução contínua, a segunda opção nos parece mais conveniente.

Uma vez que tal resultado utiliza um método de comparação envolvendo os conceitos de sub e super-soluções do problema de Cauchy 4.9 e ainda o conceito de função quase-monótona, antes de enunciá-lo apresentamos as seguintes definições, sendo que a primeira delas apresenta o conceito de solução que estamos interessados no contexto do teorema devido a Pao,

Definição 39 Dizemos que um par de funções $(u, v)$ com $u, v \in C\left([0, \bar{t}] \times \mathbb{R}^{2}\right) \cap C^{1,2}\left((0, \bar{t}] \times \mathbb{R}^{2}\right)$, onde $C^{1,2}\left((0, \bar{t}] \times \mathbb{R}^{2}\right)$ denota o conjunto das funções continuamente diferenciáveis em $t$ e com derivadas contínuas até a segunda ordem em $(x, y)$ para todo $(t, x, y) \in(0, \bar{t}] \times \mathbb{R}^{2}$, é uma solução de 4.9 se existirem constantes $a_{i}, b_{i}$, com $i=1,2$ e $b_{i}<\frac{1}{4 \bar{t}}$ tais que as seguintes condições são satisfeitas

1. $u_{t}-D_{1} \Delta u=f_{1}(u, v)$

2. $v_{t}-D_{2} \Delta v=f_{2}(u, v)$

3. $|u(t, x, y)| \leq a_{1} e^{b_{1}|(x, y)|^{2}}, \quad|v(t, x, y)| \leq a_{2} e^{b_{2}|(x, y)|^{2}}$, quando $|(x, y)| \rightarrow \infty$, com $t>0$.

Definição 40 Seja $f(z)$ uma função definida em $\mathbb{R}^{n}$ tomando valores também em $\mathbb{R}^{n}$. Reordenando as coordenadas de $z=\left(z_{1}, \cdots, z_{n}\right)$ em componentes na forma $z=\left(z_{i},[z]_{c_{i}},[z]_{d_{i}}\right)$, dizemos que $f$ tem a propriedade da quase-monotonicidade mista se existirem, para cada $i=1, \cdots, n$, inteiros não negativos $c_{i}, d_{i}$ tais que $c_{i}+d_{i}+1=n$ e $f_{i}\left(z_{i},[z]_{c_{i}},[z]_{d_{i}}\right)$ é não-decrescente em relação as componentes de $[z]_{c_{i}}$ e não-crescente em relação as componentes de $[z]_{d_{i}}$, para todo $z \in \mathbb{R}^{n}$. Em particular, $f$ é dita quase-monótona não-decrescente (não-crescente) se $c_{i}=0\left(d_{i}=0\right)$ para todo $i$.

Definição 41 Um par de funções $\left(u^{*}, v^{*}\right),\left(u_{*}, v_{*}\right)$ com $u^{*}, v^{*}, u_{*}, v_{*} \in C\left([0, \bar{t}] \times \mathbb{R}^{2}\right) \cap C^{1,2}((0, \bar{t}] \times$ $\left.\mathbb{R}^{2}\right)$, é dito sub e super-soluções acopladas de 4.9 se $\left(u^{*}, v^{*}\right) \geq\left(u_{*}, v_{*}\right)^{7}$ e as seguintes condições são satisfeitas

$1\left(u^{*}\right)_{t}-D_{1} \Delta u^{*} \geq f_{1}\left(u^{*}, v^{*}\right)$

\footnotetext{
${ }^{7} \mathrm{~A}$ desigualdade $\left(u^{*}, v^{*}\right) \geq\left(u_{*}, v_{*}\right)$ significa que $u^{*}(t, x, y) \geq u_{*}(t, x, y)$ e $v^{*}(t, x, y) \geq v_{*}(t, x, y)$, para todo $(t, x, y) \in[0, \bar{t}] \times \mathbb{R}^{2}$
} 
$\mathbf{2}\left(v^{*}\right)_{t}-D_{2} \Delta v^{*} \geq f_{2}\left(u^{*}, v^{*}\right)$

$3\left(u_{*}\right)_{t}-D_{1} \Delta u_{*} \leq f_{1}\left(u_{*}, v_{*}\right)$

$4\left(v_{*}\right)_{t}-D_{2} \Delta v_{*} \leq f_{2}\left(u_{*}, v_{*}\right)$,

e se, além disso, existirem constantes $a_{i}, b_{i}$, com $i=1,2$ e $b_{i}<\frac{1}{4 \bar{t}}$ tais que

$\mathbf{5}\left|u^{*}(t, x, y)\right| \leq a_{1} e^{b_{1}|(x, y)|^{2}}, \quad\left|v^{*}(t, x, y)\right| \leq a_{2} e^{b_{2}|(x, y)|^{2}}$

$6\left|u_{*}(t, x, y)\right| \leq a_{1} e^{b_{1}|(x, y)|^{2}},\left|v_{*}(t, x, y)\right| \leq a_{2} e^{b_{2}|(x, y)|^{2}}$, quando $|(x, y)| \rightarrow \infty$, com $t>0$.

Teorema 31 [C. V. Pao, [34]] Seja $\left(u^{*}, v^{*}\right),\left(u_{*}, v_{*}\right)$ um par de sub e super-soluções acopladas de 4.9 limitadas em $(0, \bar{t}] \times \mathbb{R}^{2}$. Sejam ainda $f_{1}$ e $f_{2}$ funções localmente Hölder contínuas com constante $\alpha$, definidas em $C\left([0, \bar{t}] \times \mathbb{R}^{2}\right) \times C\left([0, \bar{t}] \times \mathbb{R}^{2}\right)$, de classe $C^{2+\alpha}$. Além disso, se

$$
R=\left\{(u, v) \in C\left([0, \bar{t}] \times \mathbb{R}^{2}\right) \times C\left([0, \bar{t}] \times \mathbb{R}^{2}\right):\left(u_{*}, v_{*}\right) \leq(u, v) \leq\left(u^{*}, v^{*}\right)\right\}
$$

suponha que as seguintes afirmações sejam válidas

1. as funções $f_{1}$ e $f_{2}$ são lispchitzianas em $R$

2. existe $\left(u_{\sharp}, v_{\sharp}\right) \in R$ e constantes positivas $\alpha_{i}, \beta_{i}$, com $i=1,2$ e $\beta_{i}<\frac{1}{4 \bar{t}}$, tais que

$$
\left|f_{i}\left(u_{\sharp}, v_{\sharp}\right)\right| \leq \alpha_{i} e^{\beta_{i}|(x, y)|^{2}}, \quad \operatorname{comi} i=1,2,
$$

quando $|(x, y)| \rightarrow \infty$, com $t \geq 0$, para $i=1,2$.

3. $f$ satisfaz a propriedade da quase-monotonicidade.

Então o problema de Cauchy 4.9, com $\left(u_{0}, v_{0}\right) \in R$, tem única solução $(u, v)$. Além disso, tal solução é limitada pela região $R$.

Nosso próximo passo será verificar as hipóteses do teorema acima para o sistema de RosenzweigMacArthur. Vamos então procurar um par de sub e super-soluções, $\left(u^{*}, v^{*}\right),\left(u_{*}, v_{*}\right)$, para 4.13 $\left(u^{*}, v^{*}\right),\left(u_{*}, v_{*}\right)$, com a condição inicial

$$
u(0)=u_{0}, \quad v(0)=v_{0},
$$

de modo que as afirmações 1, 2 e 3 se verifiquem para a região $R$ definida acima.

Considere, por exemplo, o seguinte par de soluções constantes

$$
\begin{array}{r}
\left(u^{*}, v^{*}\right)=\left(M, \frac{k \mu}{\sigma-\mu}\right) \\
\left(u_{*}, v_{*}\right)=(0,0),
\end{array}
$$


onde $\frac{k \mu}{\sigma-\mu}<1$ e $M$ uma constante positiva qualquer. Observamos inicialmente que as condições 1-4 da definição 41 podem ser facilmente verificadas. Além disso, as não-linearidades $f_{1}$ e $f_{2}$ são de classe $C^{1}$ e limitadas na região $R$, sendo, portanto, funções globalmente lipschitzianas nessa mesma região.

Já os itens 5 e 6 da definição 41 e a afirmação 2 do teorema 31 seguem, respectivamente, do fato que as funções $u^{*}, v^{*}, u_{*}$ e $v_{*}$ são constantes e $f_{1}, f_{2}$ são limitadas em $R$. A fim de verificarmos a afirmação 2 do teorema anterior, consideramos inicialmente a função $f_{1}$, que pode ser escrita da seguinte forma

$$
f_{1}=u\left(\frac{\sigma v}{k+v}-\mu\right) .
$$

De onde segue que $f_{1}$ é não-decrescente em relação a $u$ e a $v$, na região $R$. Considerando agora a função $f_{2}$, obtemos, derivando em relação a $u$ que ela é não-crescente em relação a esta mesma variável.

Uma vez verificadas todas as hipóteses do teorema 31, podemos concluir que o modelo de Rosenzweig-MacArthur sujeito a condições iniciais restritas a região $R$ (ver 4.1) possui uma única solução. Mais ainda, tal solução é limitada pela região ${ }^{8} R$ e está, portanto, definida para todo $t>0$.

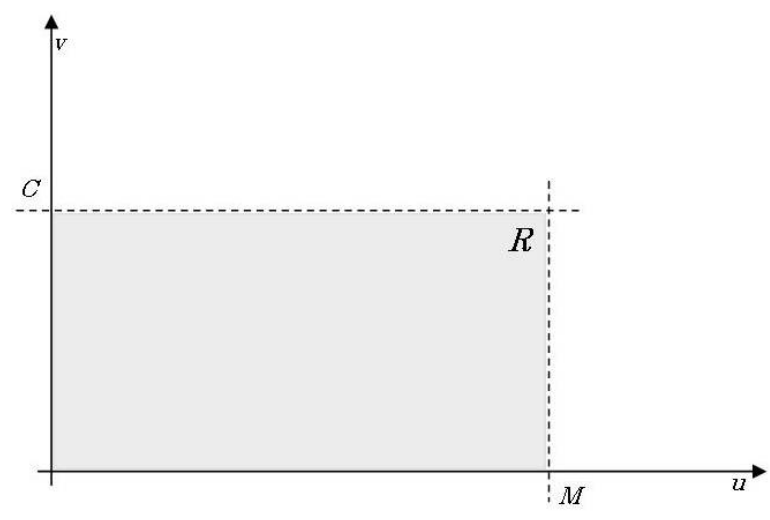

Figura 4.1: Região invariante $R \operatorname{com} C=\frac{k \mu}{\sigma-\mu}$.

Garantida a existência e unicidade de soluções de 4.13-4.15 na região

$$
R=\left\{(u, v) \in C\left([0, \bar{t}] \times \mathbb{R}^{2}\right) \times C\left([0, \bar{t}] \times \mathbb{R}^{2}\right):(0,0) \leq(u, v) \leq\left(M, \frac{k \mu}{\sigma-\mu}\right)\right\}
$$

onde $\frac{k \mu}{\sigma-\mu}<1$, nosso próximo passo será encontrar, nessa mesma região, uma particular solução, que deve ser do tipo ondas viajantes periódicas. Iremos supor que as constantes $\alpha, \sigma, k$ e $\mu$ são positivas e estamos ainda considerando soluções $(u, v)$ com ambas coordenadas estritamente

\footnotetext{
${ }^{8}$ Esse argumento de invariância, para o caso em que as funções que limitam a solução são contantes, é bastante utilizado na literatura e conhecido como retângulos invariantes. Sugerimos [10] para uma abordagem envolvendo equações de reação-difusão e [36] para outra envolvendo sistemas parabólicos em domínios limitados.
} 
positivas. Observamos inicialmente que podemos re-escrever o sistema 4.13 da seguinte forma

$$
\begin{gathered}
u_{t}=D_{1} \Delta u+f_{1}(u, v) \\
v_{t}=D_{2} \Delta v+f_{2}(u, v) .
\end{gathered}
$$

Faremos os cálculos com parâmetros arbitrários e em seguida exibiremos valores fixos, para os quais se verifica a existência de ondas viajantes periódicas.

Como vimos na seção 2.2 , a relação entre espaço e tempo nesse tipo de solução significa matematicamente que as funções $u$ e $v$ são na verdade funções de uma única variável $\mathbf{n} \cdot p+c t$, onde $p=(x, y) \in \mathbb{R}^{2}$, $c$ é uma constante positiva e $\mathbf{n}$ um vetor unitário. Devem existir então funções $2 \pi$-periódicas $U$ e $V$ tais que

$$
\begin{aligned}
& u(p, t)=U(\mathbf{n} \cdot p+c t), \\
& v(p, t)=V(\mathbf{n} \cdot p+c t) .
\end{aligned}
$$

Sendo assim, 4.18 é uma solução de 4.17 se, e somente se, $U$ e $V$ satisfazem o seguinte sistema de EDO's, de ordem 2,

$$
\begin{gathered}
D_{1} n^{2} U^{\prime \prime}+c U^{\prime}+f_{1}(u, v)=0 \\
D_{2} n^{2} V^{\prime \prime}+c V^{\prime}+f_{2}(u, v)=0 .
\end{gathered}
$$

Nosso objetivo é então verificar a existência de constantes $c$ e $n^{2}$ tais que 4.19 tenha uma solução $2 \pi$-periódica. É, porém, mais conveniente escrevermos $U(\mathbf{n} \cdot p+c t)=\tilde{U}(t-\mathbf{n} \cdot p / c)$ e $V(\mathbf{n} \cdot p+c t)=\tilde{V}(t-\mathbf{n} \cdot p / c)$, onde $\tilde{U}$ e $\tilde{V}$ devem satisfazer

$$
\begin{aligned}
& \beta D_{1} \tilde{U}^{\prime \prime}+\tilde{U}^{\prime}+f_{1}(u, v)=0 \\
& \beta D_{2} \tilde{V}^{\prime \prime}+\tilde{V}^{\prime}+f_{2}(u, v)=0
\end{aligned}
$$

para alguma constante positiva $\beta$. Tais funções devem ainda ser periódicas de período $T$, para algum $T \in \mathbb{R}$. E então, voltaremos a formulação original 4.19 , definindo $c=\frac{2 \pi}{T}$ e $n^{2}=\beta c^{2}$. É possível concluir, impondo algumas restrições a $f_{1}, f_{2}, D_{1}$ e $D_{2}$, o sistema 4.20 terá uma única solução periódica para cada coleção de valores de $\beta$ (ver [15]).

Uma vez que utilizaremos como ferramenta um teorema de bifurcação de Hopf, seguem alguns conceitos e um resultado envolvendo este tema e que, nos serão úteis adiante.

Definição 42 Considere um sistema de EDO's de dimensão 2 da forma $w_{t}=H(w)$. Dizemos que $w_{0}$ é um ponto de equilíbrio do sistema em questão se $H\left(w_{0}\right)=0$.

Teorema 32 [Teorema de Bifurcação de Hopf] Seja $w_{t}=H_{\epsilon}(w)$ um sistema de EDO's de dimensão 2 autônomo, para cada parâmetro $\epsilon$. Suponha que $H_{\epsilon}$ seja de ordem $C^{k}$, com $k \geq 3$. Assuma ainda que $w=0$ seja um ponto de equilíbrio para cada valor de $\epsilon$, e que a matriz de 
linearização do sistema em $w=0$ tenha como autovalores $\alpha(\epsilon) \pm i \beta(\epsilon)$ com $\alpha(0)=0, \beta(0) \neq 0$ $e \frac{d \alpha}{d \epsilon}(0) \neq 0$. Definindo $W=(w, \epsilon)$ e considerando o sistema $W_{t}=G(W)$ de dimensão 3, onde $G=\left(H_{\epsilon}, 0\right)$, então deverá existir uma família a um parâmetro de soluções periódicas de $W_{t}=G(W)$ em uma vizinhança $V$ de $W=0$. Além disso, não existem outras soluções periódicas em $V$.

Definição 43 Dado um sistema de EDO's da forma $w_{t}=H(w)$, uma solução periódica $w$ é dita ciclo limite quando existem duas soluções $w_{1}$ e $w_{2}$, uma pertencente ao interior $w$ e outra ao exterior, de modo que o limite de tais soluções quando $t \rightarrow+\infty$ - ou quando $t \rightarrow-\infty$ - coincide com $w$.

Observamos que, o teorema de Bifurcação de Hopf nos garante a existência de ciclos limites, uma vez que, de acordo com a definição acima, um ciclo limite é uma solução periódica $w$ tal que para alguma vizinhança de $w$ não deve existir nenhuma outra solução periódica. Ou ainda, para algum ponto $p$ na órbita de $w$, a transformação de Poincaré tem $p$ como sendo o único ponto fixo em uma vizinhança de $p$ (ver [37]).

Nosso objetivo agora é estudar o sistema

$$
\begin{gathered}
\frac{d u}{d t}=f_{1}(u, v) \\
\frac{d v}{d t}=f_{2}(u, v),
\end{gathered}
$$

com intuito de garantirmos a ocorrência de ciclo limite. É mais conveniente reescrevemos o sistema 4.13 da seguinte forma

$$
\begin{aligned}
\frac{d u}{d t} & =u F(u, v) \\
\frac{d v}{d t} & =v G(u, v),
\end{aligned}
$$

onde $F(u, v)=\frac{\sigma v}{k+v}-\mu$ e $G(u, v)=(1-v)-\frac{u}{k+v}$.

Observe que os pontos de equilíbrio do sistema 4.13 são $(0,0),(0,1)$ e $\left(\frac{(\sigma-\mu-\mu k)}{(\sigma-\mu)^{2}}, \frac{\mu k}{\sigma-\mu}\right)$. Além disso, a matriz Jacobiana de 4.21 é dada por

$$
J(u, v)=\left(\begin{array}{cc}
F(u, v)+u F_{u}(u, v) & u F_{v}(u, v) \\
v G_{u}(u, v) & G(u, v)+v G_{v}(u, v)
\end{array}\right) .
$$

Afirmamos que, se as constantes $\alpha, \sigma, k$ e $\mu$ satisfazem certas condições, então o ponto $p=\left(p_{1}, p_{2}\right)=$ $\left(\frac{(\sigma-\mu-\mu k)}{(\sigma-\mu)^{2}}, \frac{\mu k}{\sigma-\mu}\right)$ caracteriza um equilíbrio instável e, mais ainda, é verificada a ocorrência de ciclo limite numa vizinhança de $p$. 
De fato, a matriz Jacobiana de 4.21 no ponto $p$ é dada por

$$
J(p)=\left(\begin{array}{cc}
F(p)+p_{1} F_{u}(p) & p_{1} F_{v}(p) \\
p_{2} G_{u}(p) & G(p)+p_{2} G_{v}(p)
\end{array}\right)=\left(\begin{array}{cc}
0 & p_{1} F_{v}(p) \\
p_{2} G_{u}(p) & p_{2} G_{v}(p)
\end{array}\right),
$$

uma vez que $F(p)=G(p)=F_{u}(p)=0$. De onde segue que $\operatorname{tr} J(p)=p_{1} G_{v}(p)$ e det $J(p)=$ $-p_{1} p_{2} F_{v}(p) G_{u}(p)$. Sabemos que o comportamento do sistema 4.21 em uma vizinhança de $p$ é dado pela equação

$$
z_{t}=J(p) z
$$

e depende, portanto, dos autovalores da matriz $J(p)$. Além disso, as raízes do polinômio $\lambda^{2}-T \lambda+$ $D=0$, onde $T=\operatorname{tr} J(p), D=\operatorname{det} J(p)$, coincidem com os autovalores de $J(p)$.

Nosso objetivo é aplicar o teorema 32 onde o parâmetro do sistema é $k$. Então, podemos escrever

$$
\lambda(k)=\frac{T \pm \sqrt{T^{2}-4 D}}{2}=\alpha(k) \pm i \beta(k) .
$$

Fixando os parâmetros $\sigma=0,15$ e $\mu=0,05$, obtemos o seguinte, para $k=0,5$ :

$$
\alpha(0,5)=0, \quad \beta(0,5) \neq 0 .
$$

Mais ainda, sendo

$$
\alpha(k)=\frac{T}{2}=\frac{1}{2}\left[-\frac{\sigma^{23}}{\sigma-\mu}-\frac{\mu^{2} k^{2}}{\sigma-\mu}-\frac{k(\sigma+\mu) \mu}{\sigma-\mu}\right],
$$

derivando em relação a $k$ para os valores de $\sigma$ e $\mu$ fixados acima, obtemos que $\frac{d \alpha}{d k}(0,5) \neq 0$. Concluímos assim que, para $\sigma=0,15$ e $\mu=0,05$, existe um valor crítico de $k$, para o qual o sistema tem uma bifurcação de Hopf, apresentando um ciclo limite estável para valores de $k$ menores que o valor crítico $^{9}$. Observamos ainda que, neste caso, $\frac{\mu k}{\sigma-\mu}<1 \mathrm{e}$, portanto, a solução encontrada pertence a região $R$ definida anteriormente.

\footnotetext{
${ }^{9} \mathrm{O}$ teorema 32 é conhecido, em alguns contextos, como Teorema de Bifurcação de Hopf. Uma outra versão, um pouco mais geral - que pode ser encontrada em [31] -, garante a existência de soluções periódicas para parâmetros diferentes de $\epsilon \neq 0$ se, além das hipóteses do teorema considerado aqui, o coeficiente de curvatura for não-nulo. Tal condição não foi verificada neste trabalho, mas é garantida em [16] para os parâmetros $\sigma, \mu$ e $k$ considerados aqui.
} 
92CAPÍTULO 4. SISTEMAS DE REAÇÃO-DIFUSÃO: APLICAÇÕES DE SEMIGRUPOS, COMPARAÇÃO E 


\section{Apêndice A}

\section{Espaços de Sobolev e potências de Bessel}

Apresentamos uma breve discussão acerca de alguns espaços de funções fornecendo a princípio algumas definições básicas da teoria das equações diferenciais, como transformada de Fourier e convolução. Passando em seguida aos conceitos e algumas propriedades das potências de Riesz e de Bessel e, por fim, definimos espaços de Sobolev e de potências de Bessel com alguns resultados envolvendo tais espaços. Para uma discussão com mais detalhes sugerimos [14], [39], [23] e [38].

\section{A.1 Definições e resultados elementares}

Introduziremos a princípio a noção de multi-indice.

Definição 44 Um vetor em $\mathbb{R}^{n}$ da forma $\alpha=\left(\alpha_{1}, \cdots, \alpha_{n}\right)$, onde cada componente é um inteiro não negativo, com $i=1,2, \cdots$, é chamada multi-indice de ordem

$$
|\alpha|=\alpha_{1}+\cdots+\alpha_{n}
$$

Além disso, se $U \subset \mathbb{R}^{n}$ e $f: U \longrightarrow \longrightarrow$, dado um multi-indice $\alpha$, definimos

$$
D^{\alpha} f(x) \doteq \frac{\partial^{|\alpha|} f(x)}{\partial x_{1}^{\alpha_{1}} \cdots \partial x_{n}^{\alpha_{n}}}=\partial_{x_{1}}^{\alpha_{1}} \cdots \partial_{x_{n}}^{\alpha_{n}}
$$

para cada $x=\left(x_{1}, \cdots, x_{n}\right) \in U$.

Antes de apresentarmos os conceitos de transformada de Fourier e convolução, assim como algumas de suas propriedades, definiremos o espaço de Schwartz e seu dual topológico, uma vez que a transformação de Fourier tem, a princípio, como domínio de definição este primeiro espaço.

Definição 45 O espaço de Schwartz $S\left(\mathbb{R}^{n}\right)=S$ é formado pelo conjunto das funções $f \in C^{\infty}\left(\mathbb{R}^{n}\right)$ que tomam valores em $\mathbb{C}$ e que são rapidamente decrescentes, ou seja, tais que

$$
\lim _{|x| \rightarrow \infty} P(x) D^{\alpha} f(x)=0
$$

para cada polinômio $P$ e cada multi-indice $\alpha$.

As funções pertencentes aos conjuntos $C_{c}^{\infty}$ ou $S$ são ditas funções teste. O conjunto dos funcionais em $C_{c}^{\infty}$ é dito o espaço das distribuições de Schwartz e é denotado por $\mathcal{D}^{\prime}\left(\mathbb{R}^{n}\right)$. Além disso, 
denotaremos por $S^{\prime}\left(\mathbb{R}^{n}\right)=S^{\prime}$ o dual topológico de $S$, ou seja, $S^{\prime}$ é formado pelos funcionais em $S$. $S^{\prime}$ é dito conjunto das distribuições temperadas.

Definição 46 Se $f \in S$ definimos a transformada de Fourier de $f$, $\hat{f}$, como sendo

$$
\hat{f}(\xi)=\int_{\mathbb{R}^{n}} e^{-i x \xi} f(x) d x, \quad \forall \xi \in \mathbb{R}^{n},
$$

onde $x \xi=\sum_{j=1}^{n} x_{j} \xi_{j}$ é o produto escalar em $\mathbb{R}^{n}$ de $x=\left(x_{1}, \cdots, x_{n}\right)$ por $\xi=\left(\xi_{1}, \cdots, \xi_{n}\right)$. Além disso, dizemos que aplicação $\mathcal{F}: f \longmapsto \hat{f}$ é a transformação de Fourier.

A definição acima pode ser estendida para o espaço $S^{\prime}$ e assim, podemos, em particular, considerar a transformada de Fourier no contexto do espaço de Banach $L_{1}\left(\mathbb{R}^{n}\right)=L_{1}$. A extensão de $\mathcal{F}$ de $S$ para $S^{\prime}$ pode ser feita através da seguinte relação

$$
\langle\hat{S}, f\rangle=\langle S, \hat{f}\rangle
$$

para todo $f \in S$. Definiremos a seguir a inversa da transformação de Fourier, mas somente para o espaço $L_{1}$.

Definição 47 Se $\hat{f} \in L_{1}$, então dizemos que

$$
f(x)=\frac{1}{(2 \pi)^{n}} \int_{\mathbb{R}^{n}} e^{i x \xi} \hat{f}(\xi) d \xi
$$

é a transformada de Fourier inversa de $\hat{f}$. Denotaremos por $\check{g}$ a transformada inversa de uma função $g \in L_{1}$.

Definição 48 Se $f, g \in L_{1}$ então a convolução $f * g$ é definida por

$$
(f * g)=\int_{\mathbb{R}^{n}} f(x-y) g(y) d y, \quad \forall x \in \mathbb{R}^{n} .
$$

Se $f$ e $g$ são funções em $L_{1}$ então,

(i) $f * g \in L_{1} \mathrm{e}$

(ii) $(f * g)^{\wedge}(\xi)=\hat{f}(\xi) \hat{g}(\xi)$.

Além disso, se $f$ for suficientemente regular, então $\left(\partial_{j} f\right)^{\wedge}(\xi)=i \xi_{j} \hat{f}(\xi)$. Vale ainda que,

$$
\begin{aligned}
& \left(\frac{\partial^{2} f}{\partial x_{j} \partial x_{k}}\right)^{\wedge}(\xi)=\int_{\mathbb{R}^{n}} e^{-i x \xi} \frac{\partial^{2} f}{\partial x_{j} \partial x_{k}}(x) d x \\
& \quad=i \xi_{j} \int_{\mathbb{R}^{n}} e^{-i x \xi} \frac{\partial f}{\partial x_{k}}(x) d x \\
& \quad=-\xi_{j} \xi_{k} \int_{\mathbb{R}^{n}} e^{-i x \xi} f(x) d x=-\xi_{j} \xi k \hat{f}(\xi)
\end{aligned}
$$


para todo $\xi \in \mathbb{R}^{n}$ e, consequentemente,

$$
\begin{aligned}
& {[(I-\Delta) u]^{\wedge}(\xi)=\hat{u}(\xi)-(\Delta u)^{\wedge}(\xi)=\hat{u}(\xi)-\sum_{j=1}^{n}\left(\frac{\partial^{2} u}{\partial x_{j}^{2}}\right) \wedge(\xi)} \\
& =\hat{u}(\xi)+\sum_{j=1}^{n} \xi_{j}^{2} \hat{u}(\xi)=\left(1+|\xi|^{2}\right) \hat{u}(\xi) .
\end{aligned}
$$

Surge então, naturalmente, uma definição de potências do operador de Laplace, em termos da transformada de Fourier:

$$
(-\Delta)^{\frac{\alpha}{2}} f(x)=\left(|\xi|^{\alpha} \hat{f}\right)^{\wedge}(\xi)=\mathcal{F}^{-1}\left(|\xi|^{\alpha} \hat{f}(\xi)\right), \quad \forall \alpha>0, \quad \forall f \in S
$$

O lema a seguir nos garante que a transformação de Fourier $\mathcal{F}$ pode ser estendida por continuidade a uma bijeção em $L_{2}$.

Lema 17 Se $f \in L_{1} \cap L_{2}$, então $\hat{f} \in L_{2}$ e $\|\hat{f}\|_{2}=\|f\|_{2}$, onde $\|\cdot\|_{2}$ denota a norma em $L_{2}$.

Demonstração. Dada uma função $f \in L_{1} \cap L_{2}$, definimos $f_{1}(x) \doteq \overline{f(-x)}$ e $g \doteq-f_{1} * f$. Então, $\hat{g}=|\hat{f}|^{2}$. Pela definição de convolução, temos que

$$
g(x)=\int_{\mathbb{R}^{n}} \overline{f(y-x)} f(y) d y .
$$

Por outro lado, a definição de $\mathcal{F}^{-1}$ implica que

$$
g(x)=\int_{\mathbb{R}^{n}} e^{-i x \xi} \hat{g}(\xi) d \xi
$$

Tomando $x=0$ nas igualdades A.1 e A.2 obtemos $\int_{\mathbb{R}^{n}}|\hat{f}(\xi)|^{2} d \xi=\int_{\mathbb{R}^{n}}|f(y)|^{2} d y$, ou seja, $\hat{f} \in L_{2}$ $\mathrm{e}\|\hat{f}\|_{2}=\|f\|_{2}$.

\section{A.2 Potências de Riesz}

Antes de definirmos o conjunto das potências de Riesz, apresentaremos o conceito de um operador que pode ser definido de maneira natural via transformada de Fourier e que é, na verdade, uma generalização da transformada de Hilbert, dada por

$$
H(f)(x)=\frac{1}{\pi} \int_{-\infty}^{\infty} \frac{f(t)}{x-t} d t, \quad \forall f \in L_{p}
$$

para dimensões maiores. Tal operador é conhecido como transformada de Riesz e é dado pela seguinte

Definição 49 Se $f \in L_{p}$, com $1 \leq p<\infty$ então, para cada $j=1, \cdots, n$, a transformada de Riesz 
$\mathcal{R}_{j}$ de $f$ é definida por

$$
\mathcal{R}_{j}(f) x=\lim _{\epsilon \rightarrow 0} c_{n} \int_{|y| \geq \epsilon} K_{j}(y) \frac{y_{j}}{f}(x-y),
$$

onde $K_{j}(x)=c_{n} \frac{y_{j}}{|y|^{n+1}}$ é o Kernel de Riesz singular e $c_{n}=\Gamma\left(\frac{n+1}{2}\right) / \pi^{\frac{n+1}{2}}$.

Como vimos na seção anterior, se função $f \in S$ então sua transformada de Fourier $\hat{f}$ e o laplaciano de $f, \Delta f$, estão relacionados da seguinte forma

$$
(-\Delta f)^{\wedge}(\xi)=|\xi|^{2} \hat{f}(\xi)
$$

Vimos ainda que, substituindo a potência de $|\xi|$ por um $\alpha$ qualquer obtemos potências fracionárias do laplaciano,

$$
(-\Delta)^{\frac{\alpha}{2}} f(x)=\left(|\xi|^{\alpha} \hat{f}\right)^{\wedge}(\xi), \quad \forall \alpha>0
$$

Se $\alpha \in(0, n)$, então a função $|\xi|^{-\alpha}$ é localmente integrável e, portanto, é um elemento de $S^{\prime}$. Podemos assim estender a definição acima para potências negativas, ou seja, podemos definir $(-\Delta)^{-\frac{\alpha}{2}}$ para $\alpha \in(0, n)$. Para tal utilizaremos operadores integrais da forma $\mathcal{I}_{\alpha}: S \longrightarrow S^{\prime}$, conhecidos como potências de Riesz.

Definição 50 Se $f \in S$ e $\alpha \in(0, n)$ então definimos

$$
(-\Delta)^{-\frac{\alpha}{2}}=\mathcal{I}_{\alpha} f
$$

onde

$$
\left(\mathcal{I}_{\alpha} f\right)(x) \doteq \frac{1}{\gamma(\alpha)} \int_{\mathbb{R}^{n}}|x-y|^{-n+\alpha} f(y) d y
$$

$e \gamma(\alpha)=\pi^{\frac{n}{2}} 2^{\alpha} \Gamma\left(\frac{\alpha}{2}\right) / \Gamma\left(\frac{n-\alpha}{2}\right)$

Para cada $\alpha \in(0, n)$, definimos a função $I_{\alpha}$, conhecida como Kernel de Riesz e dada por

$$
I_{\alpha}(x)=\frac{|x|^{\alpha-n}}{\gamma(\alpha)},
$$

onde $\gamma(\alpha)=\left(\pi^{\frac{n}{2}} 2^{\alpha} \Gamma\left(\frac{\alpha}{2}\right)\right) / \Gamma\left(\frac{n-\alpha}{2}\right)$. Assim, é possível obter a seguinte representação para as potências de Riesz $\mathcal{I}_{\alpha}$ :

$$
\mathcal{I}_{\alpha} f(x)=\left(I_{\alpha} * f\right)(x), \quad \forall \alpha \in(0, n) .
$$

Mais ainda, dada uma função $f$ em $S$, ela pode ser escrita como uma potência de Riesz. É suficiente tomar $g=(-\Delta)^{\frac{\alpha}{2}} f$ e então $f(x)=\mathcal{I}_{\alpha} g(x)$.

\section{A.3 Espaços de Sobolev}

Os espaços de Sobolev constituem uma poderosa ferramenta da análise funcional, principalmente no que diz respeito à sua relação com as EDP's. A definição de tais espaços caracteriza uma 
importante relação entre funções em $C_{c}^{\infty}$ e suas derivadas parciais. Mas o conceito de derivadas parciais que está envolvido é dado por uma noção mais geral que aquela usual e em termos da teoria da distribuições, como vemos na seguinte

Definição 51 Sejam $f$ e $g$ duas funções localmente integráveis em $\mathbb{R}^{n}$. Dado um multi-indice $\alpha$, dizemos que $D^{\alpha} f=g$ no sentido fraco (ou das distribuições), se

$$
\int_{\mathbb{R}^{n}} f(x) D^{\alpha} \varphi(x) d x=(-1)^{|\alpha|} \int_{\mathbb{R}^{n}} g(x) \varphi(x) d x, \quad \forall \varphi \in C_{c}^{\infty} .
$$

Observamos que, se $f$ possui todas as derivadas parciais até a ordem $|\alpha|$ contínuas e $D^{\alpha} f=g$ no sentido usual, então, aplicando integração por partes, obtemos A.4.

Definição 52 Para cada inteiro não negativo $m$, o espaço de Sobolev $W^{m, p}\left(\mathbb{R}^{n}\right)=W^{m, p}$ é definido por

$$
W^{m, p}=\left\{f \in L_{p}: \exists D^{\alpha} f \text { e } D^{\alpha} f \in L_{p}, \forall \alpha \text { com }|\alpha| \leq m\right\}
$$

onde $D^{\alpha}$ se refere a derivada parcial de $f$ no sentido fraco.

O espaço de Sobolev $W^{m, p}$ com a norma

$$
\|f\|_{W^{m, p}}=\sum_{|\alpha| \leq m}\left(\int_{\mathbb{R}^{n}}\left|D^{\alpha} f\right|^{p}\right)^{\frac{1}{p}}
$$

é um espaço completo (ver [38]). Geralmente na literatura encontra-se a seguinte notação

$$
W^{m, 2}=H^{m}
$$

Definição 53 Definimos o espaço $W_{0}^{m, p}$ como sendo o fecho de $C_{c}^{\infty}\left(\mathbb{R}^{n}\right)$ em $W^{m, p}\left(\mathbb{R}^{n}\right)$.

Observamos ainda que é comum na literatura a seguinte notação

$$
W_{0}^{m, 2}=H_{0}^{m}
$$

Para mais detalhes acerca de tais espaços ver [21].

O próximo resultado fornece uma caracterização alternativa dos espaços de Sobolev $W^{m, p}$, que não envolve a noção de derivada parcial no sentido fraco e cuja demonstração pode ser encontrada em [38].

Teorema 33 Seja $f \in L_{p}$, com $1 \leq p<\infty$. Então $f \in W^{m, p}$ se, e somente se, existir uma seqüência $\left\{\varphi_{n}\right\}$ de funções em $C_{c}^{\infty}$ tal que

1. $\left\{\varphi_{n}\right\}$ converge para $f$ em $L_{p}$ 
2. para cada $\alpha$ de ordem $|\alpha| \leq m,\left\{D^{\alpha} \varphi_{n}\right\}^{1}$ converge na norma de $L_{p}$.

Ao passo que os espaços $C^{k}\left(\mathbb{R}^{n}\right)$ de certa forma medem a suavidade de funções em $\mathbb{R}^{n}$ em termos de suas derivadas parciais no sentido usual, os espaços de Sobolev estabelece uma classificação destas mesmas funções, porém em termos das derivadas parciais no contexto da definição 42.

Os espaços das funções em $\mathbb{R}^{n} k$-vezes continuamente diferenciáveis estão relacionados por imersões da forma

$$
C^{1}\left(\mathbb{R}^{n}\right) \supset C^{2}\left(\mathbb{R}^{n}\right) \supset \cdots \supset C^{k}\left(\mathbb{R}^{n}\right) \supset \cdots
$$

Uma pergunta natural seria se os espaços $W^{\alpha, p}$ possuem uma propriedade semelhante ou, ainda mais, se existe alguma relação envolvendo também os espaços $L_{p}$. O próximo resultado, devido a Sobolev, responde parte destas perguntas.

Teorema 34 (Teorema de Sobolev) Sejam $\alpha>0$ e $1<p<\infty$. São válidas as seguintes afirmações:

1. quando $\alpha p<n$, se $p \leq q \leq p^{*}$, onde $\frac{1}{p^{*}}=\frac{1}{p}-\frac{\alpha}{n}$, então $W^{\alpha, p}\left(\mathbb{R}^{n}\right) \subset L_{q}\left(\mathbb{R}^{n}\right)$;

2. quando $\alpha p=n$, se $p \leq q<\infty$, então $W^{\alpha, p}\left(\mathbb{R}^{n}\right) \subset L_{q}\left(\mathbb{R}^{n}\right)$;

3. quando $\alpha p>n$, então $W^{\alpha, p}\left(\mathbb{R}^{n}\right) \subset C\left(\mathbb{R}^{n}\right)$.

Diferentes abordagens deste teorema podem ser encontradas em [21], [38] e [13].

\section{A.4 Potências de Bessel}

A noção do espaço de potências de Bessel surgiu da necessidade de generalizar a definição de espaços de Sobolev $W^{m, p}$ para o caso em que $m$ não é inteiro. Além disso, surgem alguns problemas envolvendo a representação apresentada da seção A.2 de uma função $f \in S$ em termos das potências de Riesz. Tais problemas surgem, por exemplo, do fato de $f \longmapsto I_{\alpha} * f$ não ser um operador limitado em $L_{p}$ (ver [38]). Outra questão que pode ser levantada, envolve o comportamento do Kernel de Riesz no infinito. Ao passo em que

$$
\frac{|x|^{\alpha-n}}{\gamma(\alpha)}
$$

é adequado para $x$ numa vizinhança da origem, ele não se aplica da mesma maneira para o caso em que $|x|$ é suficientemente grande (i.e. $|x| \rightarrow \infty$ ).

Uma solução seria substituir as potências de Riesz $\mathcal{I}_{\alpha}$ por uma função que tivesse a mesma singularidade na origem, mas que apresentasse um rápido decaimento no infinito. Existem várias maneiras de se obter uma função com tais aspectos. Podemos citar por exemplo a função $\mathcal{I}_{\alpha} e^{-|x|}$. Há porém, algumas vantagens em proceder via transformada de Fourier e substituir $(-\Delta)^{-\frac{\alpha}{2}}$ por $(I-\Delta)^{-\frac{\alpha}{2}}$.

\footnotetext{
${ }^{1}$ Estamos nos referindo a derivada parcial no sentido usual, uma vez que $\left\{\varphi_{n}\right\} \in C_{c}^{\infty}$. O que na verdade ocorre é que, como vimos, neste caso os conceitos de derivada no sentido fraco e usual coincidem.
} 
Definição 54 Definimos as potências de Bessel $\mathcal{J}_{\alpha}$ por

$$
\mathcal{J}_{\alpha}=(I-\Delta)^{-\frac{\alpha}{2}}
$$

Para cada $\alpha \in \mathbb{R}$, define-se o Kernel de Bessel $G_{\alpha}$ por

$$
G_{\alpha}(x)=\frac{1}{(4 \pi)^{\frac{n}{2}}} \frac{1}{\Gamma\left(\frac{\alpha}{2}\right)} \int_{0}^{\infty} t^{\frac{\alpha-n}{2}} e^{-\frac{\pi|x|^{2}}{t}} e^{-\frac{t}{4 \pi}} \frac{d t}{t}
$$

Segue uma proposição acerca das propriedades do Kernel $\alpha \in \mathbb{R}$.

Proposição 7 Considere $G_{\alpha}$ como afoi definido acima. Então

1. para cada $\alpha>0, G_{\alpha} \in L_{1}\left(\mathbb{R}^{n}\right)$

2. $\hat{G}_{\alpha}(\xi)=\left(1+|\xi|^{2}\right)^{-\frac{\alpha}{2}}$

Estudaremos agora algumas propriedades da família $\left\{\mathcal{J}_{\alpha}\right\}_{\alpha}$. Para cada $\alpha \in \mathbb{R}$ e $f \in L_{p}\left(\mathbb{R}^{n}\right)$, podemos, de maneira análoga ao que foi feito para potências de Riesz, representar $\mathcal{J}_{\alpha}$ da seguinte forma

$$
\mathcal{J}_{\alpha}(f)=G_{\alpha} * f
$$

A convolução acima está bem definida, uma vez que $\left\|G_{\alpha}\right\|_{1}=\int_{\mathbb{R}^{n}} G_{\alpha}(x) d x=1$. Além disso, são válidas as seguintes afirmações

1. $\mathrm{J}_{\alpha} \mathcal{J}_{\beta}=\mathcal{J}_{\alpha+\beta}, \quad \forall \alpha, \beta \in \mathbb{R}$

2. $\mathrm{J}_{\alpha}^{-1}=\mathcal{J}_{-\alpha}, \quad \forall \alpha, \beta \in \mathbb{R}$

3. $\left\|\mathcal{J}_{\alpha}(f)\right\|_{p} \leq\|f\|_{p}, \quad \forall 1 \leq \infty, \quad \forall f \in L_{p}\left(\mathbb{R}^{n}\right)$.

Definição 55 O conjunto $\mathcal{L}_{\alpha}^{p}$ dado por

$$
\mathcal{L}_{\alpha}^{p}=\left\{f: f=G_{\alpha} * g, g \in L_{p}\left(\mathbb{R}^{n}\right)\right\}
$$

é conhecido como espaço das potências de Bessel, com $\alpha \in \mathbb{R}$.

Podemos dizer, simbolicamente, que $\mathcal{L}_{\alpha}^{p}=\mathcal{J}_{\alpha}\left(L_{p}\left(\mathbb{R}^{n}\right)\right)$, para $1 \leq p \leq \infty$ e $\alpha \in \mathbb{R}$.

A norma em $\mathcal{L}_{\alpha}^{p}$, denotado por $\|\cdot\|_{p, \alpha}$ é definida da seguinte forma,

$$
\|f\|_{p, \alpha}=\|g\|_{p}, \text { se } f=\mathcal{J}_{\alpha}(g) \text {. }
$$

Veremos que a norma em $\mathcal{L}_{\alpha}^{p}$, assim definida, é consistente. Dados $g_{1}$ e $g_{2}$ em $L_{p}\left(\mathbb{R}^{n}\right)$, nosso objetivo é então provar que $\mathcal{J}_{\alpha}\left(g_{1}\right)=\mathcal{J}_{\alpha}\left(g_{2}\right)$ implica que $g_{1}=g_{2}$. Observamos a princípio que, se $\varphi \in S$ então

$$
\int \mathcal{J}_{\alpha}\left(g_{1}\right) \varphi(x) d x=\int G_{\alpha} * g_{1} \varphi(x) d x=\iint G_{\alpha}(x-y) g_{1}(y) \varphi(x) d x d y=\int g_{1} \mathcal{J}_{\alpha}(\varphi) d x .
$$


Uma vez que $\mathcal{J}_{\alpha}\left(g_{1}\right)=\mathcal{J}_{\alpha}\left(g_{2}\right)$, obtemos, pelo teorema de Fubini, que

$$
0=\int_{\mathbb{R}^{n}} \mathcal{J}_{\alpha}\left(g_{1}\right)-\int_{\mathbb{R}^{n}} \mathcal{J}_{\alpha}\left(g_{2}\right)=\int_{\mathbb{R}^{n}}\left(g_{1}-g_{2}\right) \mathcal{J}_{\alpha}(\varphi), \quad \forall \varphi \in S
$$

Se provarmos que $\mathcal{J}_{\alpha}: S \longrightarrow S$ é uma bijeção, então, para cada $\psi \in S$, existiria $\varphi \in S$ tal que $\mathcal{J}_{\alpha}(\varphi)=\psi$, e portanto, teríamos

$$
\int\left(g_{1}-g_{2}\right) \psi d x=0, \quad \forall \psi \in S
$$

de onde obteríamos que $g_{1}-g_{2}=0$. Considere então algum elemento $\psi$ de $S$ e defina $\hat{\varphi}(x)=$ $\hat{\psi}(x)\left(1+|x|^{2}\right)^{-\frac{\alpha}{2}}$. Sendo $\hat{\psi}$ um elemento de $S$, deve-se ter $\hat{\varphi} \in S$ e, portanto, $\varphi \in S$. Além disso, como $G_{\alpha}=\mathcal{F}^{-1}\left(\left(1+|x|^{2}\right)^{-\frac{\alpha}{2}}\right)$ e $\hat{\psi}(x)=\hat{\varphi}(x)\left(1+|x|^{2}\right)^{-\frac{\alpha}{2}}$, obtemos

$$
\mathcal{J}_{\alpha}(\varphi)=\psi
$$

Observamos ainda que, sendo $\mathcal{J}_{\alpha}: S \longrightarrow S$, podemos escrever cada elemento $f$ de $S$ como potências de Bessel. Basta tomar $g=\mathcal{J}_{-\alpha}(f)$ e, então $f=\mathcal{J}_{\alpha}(g)$.

Apresentamos a seguir um importante resultado, que não será demonstrado aqui (ver [6] e [38]), acerca da relação entre os espaços de Sobolev $W^{, p}\left(\mathbb{R}^{n}\right)$ e o espaço das potências de Bessel $\mathcal{L}_{\alpha}^{p}\left(\mathbb{R}^{n}\right)$. Este resultado nos garante que tais espaços coincidem, para cada $p \in(1, \infty)$, para o caso em que as potências $\alpha$ são inteiras.

Teorema 35 [A. P. Calderón] Suponha que $m$ seja um inteiro positivo e $p \in(1, \infty)$. Então

$$
\mathcal{L}_{m}^{p}\left(\mathbb{R}^{n}\right)=W^{m, p}\left(\mathbb{R}^{n}\right)
$$

com normas equivalentes, ou seja, existem constantes a e b tais que

$$
a\|f\|_{p, m} \leq\|f\|_{W^{m, p}} \leq b\|f\|_{p, m} .
$$

O mesmo não ocorre quando $p=1$ ou $p=\infty$. As relações de inclusão entre os espaços de Sobolev e de potências de Bessel para estes dois casos extremos são apresentadas a seguir e discutidas em [38].

Teorema 36 Considere os espaços $\mathcal{L}_{m}^{p}\left(\mathbb{R}^{n}\right)$ e $W^{m, p}\left(\mathbb{R}^{n}\right)$. São válidas as seguintes afirmações:

1. $W^{m, p}(\mathbb{R})=\mathcal{L}_{m}^{p}(\mathbb{R})$ quando $m$ é par e $p=1$ ou $\infty$.

2. Se $n>1$ então $W^{m, p}(\mathbb{R}) \subset \mathcal{L}_{m}^{p}(\mathbb{R})$, quando $m$ é par e $p=1$ ou $\infty$.

3. Se $m$ é impar então, para todo $n, W^{m, p}(\mathbb{R}) \nsubseteq \mathcal{L}_{m}^{p}(\mathbb{R})$ e $\mathcal{L}_{m}^{p}(\mathbb{R}) \nsubseteq W^{m, p}(\mathbb{R})$. 


\section{Referências Bibliográficas}

[1] V. Volpert e V. Volpert A. Volpert, Traveling wave solutions of parabolic systems, primeira ed., Clarendon Press, Oxford, 1994. 2, 18, 19, 20

[2] H. Amann, Linear and quasilinear parabolic problems. vol. 1, abstract linear theory, primeira ed., Birkhäuser, Basiléia, 1995. 62

[3] A. Belleni-Morante, Applied semigroups and evolution equations, primeira ed., Clarendon Press, Oxford, 1979. 62

[4] H. Brézis, Análisis functional, teoría y aplicaciones, primeira ed., Alianza Editorial, Madrid, 1984. 2

[5] N.F. Britton, Essential mathematical biology, primeira ed., Springer, Nova Iorque, 2003. 2

[6] A.P. Calderón, Lebesgue spaces of differentiable functions and distributions, in. partial differential equations., primeira ed., Proc. Sympos. Pure Math. AMS, Providence, 1961. 100

[7] A. N. Carvalho, Equações parabólicas semilineares, Preprint, ICMC-USP (2001). 2, 70

[8] _ Análise funcional II, Preprint, ICMC-USP (2009). 2

[9] R. Czaja, Differential equations with sectorial operators, primeira ed., Wydawnictwo Uniwersytetu Ślaskiego, Katowice, 2002. 2, 29, 82

[10] M. H. Protter e H. F. Weinberger, Maximum principles in differential equations, primeira ed., Prentice-Hall, Englewood Cliffs, 1967. 88

[11] J. Hale e H. Koçak, Dynamics and bifurcations, primeira ed., Springer, Nova Iorque, 1991. 2, 10

[12] N. Dunford e J. Schwartz, Linear operators, part I, general theory, primeira ed., Wiley, Nova Iorque, 1957. 21

[13] R.A. Adams e J.J.F. Fournier, Sobolev spaces, segunda ed., PAMS, Elsevier, Kidlington, 2003. 98

[14] D. R. Adams e L. I. Hedberg, Function spaces and potential theory, primeira ed., GMW 314, Springer, Berlim, 1996. 2, 93

[15] N. Kopell e L.N. Howard, Plane wave solutions to reaction-diffusion equations., Stud. Appl. Math. 52 (1973), 291-328. 84, 89

[16] J. A. Sherratt e M.J. Smith, Periodic travelling waves in cyclic populations: field studies and reaction-diffusion models, Journal of Royal Society 220 (2008), 13-41. 2, 5, 14, 77, 85, 91 
[17] M. Renardy e R. C. Rogers, An introduction to partial differential equations, primeira ed., Texts in Applied Mathematics 13, Springer, Nova-Iorque, 1993. 2

[18] K. Hoffman e R. Kunze, Linear algebra, primeira ed., Prentice-Hall, Englewood Cliffs, 1961. 22

[19] M.L. Rosenzweig e R.H. MacArthur, Graphical representation and stability conditions of predator-prey interactions, Am. Nat. 97 (1963), 209-223. 85

[20] G.R. Sell e Y. You, Dynamics of evolutionary equations, primeira ed., Applied Mathematical Sciences 143, Springer, Nova Iorque, 2002. 2, 30

[21] L. Evans, Partial differential equations, primeira ed., American Mathematical Society, Providence, 1991. 6, 97, 98

[22] A. Friedman, Partial differential equations, primeira ed., Holt, Rinehart Winston, Nova Iorque, 1969. 52,78

[23] J. Goldstein, Semigroups of linear operators and applications, primeira ed., Oxford University Press, Nova Iorque, 1985. 2, 47, 76, 93

[24] P. Grindrod, Patterns and waves : the theory and applications of reaction-diffusion equations, primeira ed., Clarendon Press, Oxford, 1991. 2

[25] J. Hale, Ordinary differential equations, segunda ed., Krieger, Huntington, 1980. 10

[26] C. S. Hönig, Aplicações da topologia à análise, primeira ed., IMPA, Rio de Janeiro, 1976. 73

[27] D. Henry, Geometric theory of semilinear parabolic equations, primeira ed., Lecture Notes in Mathematics 840, Springer, Berlim, 1981. 2

[28] F. John, Partial differential equations, quarta ed., Springer, Nova Iorque, 1982. 6

[29] T. Kato, Perturbation theory for linear operators, segunda ed., Springer, Nova Iorque, 1984. 21

[30] E. Kreyszig, Introductory functional analysis with applications, primeira ed., Wiley, Nova Iorque, 1978. 23, 28

[31] A. I. Mees, Dynamics of feedback systems, primeira ed., John Wiley Sons, Nova Iorque, 1981. 91

[32] R. E. Megginson, An introduction to banach space theory, primeira ed., Springer, Nova-Iorque, 1998. 23,30

[33] M. Miklavčič, Applied functional analysis and partial differential equations, primeira ed., World Scientific Publishing, Londres, 1998. 2, 62

[34] C.V. Pao, Parabolic systems in unbounded domains I. existence and dynamics, Journal of Mathematical Analysis and Applications 217 (1998), 129-160. 2, 87

[35] A. Pazy, Semigroups of linear operators and applications to partial differential equations, primeira ed., Applied Mathematical Sciences 44, Springer, Nova Iorque, 1983. 2, 70, 73 
[36] J. Smoller, Shock-waves and reaction-diffusion equations, gmw, 258, primeira ed., Springer, Nova Iorque, 1983. 2, 7, 14, 88

[37] J. Sotomayor, Lições de equações diferenciais ordinárias, primeira ed., IMPA, Rio de Janeiro, 1979. 10,90

[38] E. M. Stein, Singular integrals and differentiability properties of functions, primeira ed., Princeton University Press, Princeton, 1970. 2, 83, 93, 97, 98, 100

[39] H. Triebel, Theory of function spaces II, primeira ed., Birkhäuser, Basiléia, 1992. 93 


\section{Índice Remissivo}

$\sigma$-álgebra de Borel, 37

Aplicação

dualidade, 30

Autovalores, 12

Bifurcação de Hopf, 10

Ciclo limite, 90

Condição de fronteira, 6 de Dirichlet, 7

de Neumann, 7

de Robin, 7

Convolução, 94

Derivada no sentido fraco, 97

Desigualdade Calderón-Zygmund, 78

Equação

do calor, 5

reação-difusão, 5

Espaço

das distribuições de Schwartz, 93

de Banach, 25

de Hilbert, 28

de Schwartz, 93

de Sobolev, 11, 97

dual, 27

Normado, 21

Espectro, 11, 22

contínuo, 22

pontual, 22

residual, 22

Função quase-monótona, 86

Funcionais lineares, 27

Gerador

infinitesimal, 35

geradores, 30
Kernel

de Bessel, 96, 99

de Riesz, 96

laplaciano, 5

Leray-Schauder, 19

Método de Comparação, 86

Multi-indice, 93

Onda viajante periódica, 13

Ondas

estacionárias, 18

periódicas, 18

planas, 18

Operador

acretivo, 30

adjunto, 27

auto-adjunto, 28

Desclocamento direito, 49

diagonal, 81

dissipativo, 30

fechável, 25

fechado, 24

Identidade, 21

setorial, 48

simétrico, 28

Ponto de equilíbrio, 89

assintoticamente estável, 9

instável, 15

instãvel, 11

Potências

de Bessel, 99

de Riesz, 96

do operador Laplace, 95

Problema de Cauchy, 6

bem posto, 9 
Resolvente, 21, 22

Retrato de fases, 14

Semigrupo, 30

analítico, 47

de contrações, 40

de operadores lineares, 21, 33

fortemente contínuo, 33

uniformemente contínuo, 33

Sistema

de Rosenzweig-MacArthur, 85

do tipo $\lambda-\omega, 84$

Solução

periódica estável, 20

Sub-solução, 86

Subconjunto denso, 21

Super-solução, 86

Teorema

de Bifurcação de Hopf, 89

de Hahn-Banach, 30

de Hille-Yosida, 40, 41

de Lumer-Phillips, 40

de Sobolev, 98

do núcleo e imagem, 22

Teoria qualitativa, 10

Transformação

de Fourier, 94

de Poincaré, 90

Transformada

de Fourier, 7, 94

de Riesz, 95

Valores próprios, 22

Variedade estável, 17

Variedade instável, 17

Vetor próprio , 22 